Florida International University FIU Digital Commons

6-1976

\title{
An analysis of hospital temperature maintenance and tray assembly systems : an industry project
}

FrankJohn DeWerff

Florida International University

DOI: $10.25148 /$ etd.FI14062255

Follow this and additional works at: https://digitalcommons.fiu.edu/etd

Part of the Hospitality Administration and Management Commons

\section{Recommended Citation}

DeWerff, Frank John, "An analysis of hospital temperature maintenance and tray assembly systems : an industry project" (1976). FIU Electronic Theses and Dissertations. 2785.

https://digitalcommons.fiu.edu/etd/2785 


\author{
AN ANALYSIS OF \\ HOSPITAL TEMPERATURE MAINTENANCE \\ AND TRAY ASSEMBLY SYSTEMS
}

\begin{abstract}
An Industry Project
Presented to the Faculty of the school of

Hotel, Food, and Iravel Services at
\end{abstract}

Florida International university in partial Fulfillment

of the Requirements for the Degrese of

Master of Science in Hotel and Food Service Manayement

by

Frank John DeWerff

June, 1976 


\section{INTRODUCTION}

Every hospital, private or public, profit or nonprofit, no matter the size, has the task of feeding its patients three times a day plus nourishments. The meals must be served during a short period of time to patients in their rooms which are spread throughout the hospital. The meals must be therapeutically balanced, attractive, the hot foods hot and the cold foods cold, and they must be nutritious. The meals in a hospital differ from those served in restaurants in that all of the courses are served at one time which means that there is greater importance placed on temperature maintenance. Considering that most hospitals offer a selective menu, the task is quite unique and challenging.

Hospitals, profit or non-profit are conscious of cost, efficiency, and patient satisfaction. Therefore, the selection of a system to assemble and deliver meals to the patient is of the utmost importance.

The purpose of this study is to identify and compare assembly and temperature maintenance and delivery systems currently available; in order to make available information for those who are planning systems and for those who are learning about these systems. 
Table of Contents

Page

Introduction . . . . . . . . . . . . . . . . i

History . . . . . . . . . . . . . . . . . 1

Prerequisites . . . . . . . . . . . . 6

Temperature Maintenance Delivery Systems . . . . . 13

Tray Assembly Systems . . . . . . . . . . . 82

Transporting ................. 102

summary . . . . . . . . . . . . . . 107

Credits for Exhibits . . . . . . . . . . . III

Bibliography .................. . 113 
Exhibits

Page

Meal-Pak .................... 15

Temp-Lock . . . . . . . . . . . . . . . . . 17

Dinex Insul-Server . . . . . . . . . . . . . . 18

Cambro Heat Keeper . . . . . . . . . . . . . 19

Heated Plate/Bowl Dispenser . . . . . . . . . . 21

Caddy T-540 Storage Rack . . . . . . . . . . . 23

Comparative Heat Retention Data. . . . . . . . . 24

Dinex Mugs and Beverage Servers . . . . . . . . . 25

Stainless Steel Insulated Beverage Server. . . . . 26

Stainless Steel Insulated Bowl . . . . . . . . . . 27

Aladdin . . . . . . . . . . . . . . . 30

Insulated Plastic Containers . . . . . . . . . . 34

Isolation Tray Service . . . . . . . . . . . . 35

Dri-Heat . . . . . . . . . . . . . . . 39

3 Piece Pellet ................ . . . . 40

Unitized Base . . . . . . . . . . . . . . . . . . 41

Heat Retention Chart . . . . . . . . . . . . . 44

Tray Delivery Cart . . . . . . . . . . . . . 45

1216 . . . . . . . . . . . . . 4 47

1216 cart......................448

Elegant Dining . . . . . . . . . . . . . . . 49

Hot and Cold Cart... . . . . . . . . . . 52

Unitray . . . . . . . . . . . . . . . . 53

Sweetheart Cart................ . 55

Drice Cart ................... . 58

Cold Cart....................... 59

Bulk Food Cart ................... 60

Microwave Oven .................. 63

Galley Station . . . . . . . . . . . . . 66

3 M Integral Heating System . . . . . . . . . 68

Food Chariot System . . . . . . . . . . . . . 70

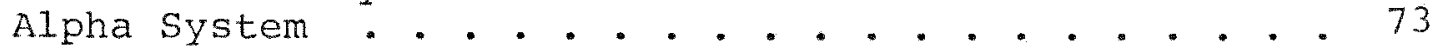

Chemetron Votator. . . . . . . . . . . . . 75

Crimsco Galley System... . . . . . . . . . . . . 78

Foster Re-Con Ovens................ . . 80

Shelley Tray Line................... . . 85

Skate wheel Conveyor . . . . . . . . . . . 87

Precision Tray Line............... . . 88 
Exhibits, Cont'd

Page

Roller Conveyor . . . . . . . . . . . . . 90

Belt Conveyor . . . . . . . . . . . . . . . 91

Tray Assembly Diagram . • • • • • • • • • • . 93

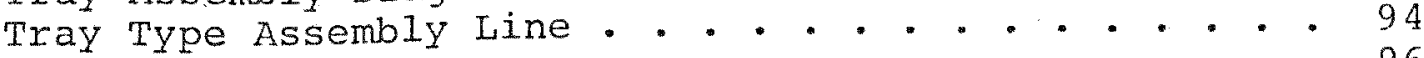

Staffing Chart . . . . . . . . . . . . . . 96

Dumb Waiter................... . 103

Cart-veyor .................... 104 


\section{ACKNOWLEDGMENTS}

The author wishes to acknowledge the assistance of William J. Morgan, Jr., Ph.D., and Bonny R. Prentiss, R.D., M.S., who served as advisors. 


\section{HISTORY}

Hospital temperature maintenance and tray assembly systems are relatively new innovations. Though hospitals have existed for centuries, little consideration had been given to serving hot nutritious meals. Being sick was often thought to be a sign of weakness. Darwin's theory of the survival of the fittest was applied to social behavior. Those who were sick were cast aside or placed in almshouses which were homes for the poor and the sick. The conditions in these houses were miserable. Only the minimal of care was given with little or no attention given to the diet as a means of therapy.

The 19th century saw the development of hospital feeding that did not kill as many patients as it helped cure. For this the world owes another great debt of gratitude to Florence Nightingale, who was the founder of dietetics as well as modern nursing.

In the diet kitchen she set up at the hospital at Scutari (now part of Istanbul) during the crimean war, she replaced the ill-cooked, ill served fare with punctual appetizing meals, even including some extra foods like soups and jellies for those who needed them. Alexis soyer, a noted chef, served as the manager and organizer of this 
first modern hospital kitchen. Mr. Soyer refused to accept inferior provisions, posted menus in full public view and submitted lists of ingredients to the medical authorities for their approval. The one thing neither he nor Miss Nightingale could do was convince the military authorities to allow them to separate meat from the bone before it was served, so that some soldiers would not get all meat, and some all bone. Military regulations would not permit it, they were told; the soldiers would just have to take their chances.

Miss Nightingale was one of the first to consider that the science and art of feeding the sick was an essential part of nursing. In her recognition of nursing she, therefore, placed great emphasis on the selection and service of food, and in all her plans for hospital reorganization, the food service was placed under the nurse-matron where it remained in Britain until very recent times. Before Miss Nightingale's developments, hospitals were simply run on a day to day basis as indicated by the accounts for the Pennsylvania Hospital of 150 years ago.

In the year 1804, milk, butter, pork, soap, and hay were produced on this hospital's ground and consumed therein. The matron, among her accomplishments made rose water for which the sum of $\$ 53$ was received. Numerous cows, calves, and pigs were sold. Patients paid the hospital for their funeral charges.... On the debit side we find the sum of $\$ 350$ paid for nine months' salary to Francis and Hannah Higgins as steward and matron. It required 1,280 pounds of candles at a cost of $\$ 182$ to furnish light, and $\$ 40$ rent was paid to pasture the cows. A year's tobacco cost $\$ 2.89$. For a hospital population of 419 
hospital cases for the year, 838 gallons of molasses, 254 gallons of brandy and spirits, 269 gallons of wine, and $24 \%$ barrels of beer were consumed, among items listed under liquors. 1

...dungeon-like aspect of the kitchen with fumes of cabbage cooking. It was poorly ventilated and dim. There was no diet kitchen and no special diets. Each morning I received a list of foods purchased by the superintendent and was told to use them as soon as possible. Coal ranges were the mode of cooking. Refrigeration was an old fashioned ice chest in which was kept five gallon cans of milk and milk was dipped from it as needed. 2

But progress was being made, and in 1924, a dietitian

in New York City described her food service in this manner:

A system of centralized food service is used for private patients. This means that the trays are practically ready to serve when they leave the kitchen. The trays are set-up with china, silver, and other appointments before time for serving the meal, and placed on food conveyors. These metal carriages, equipped with shelves, are wheeled into the kitchen and every tray is checked by the dietitian before it leaves. Hot water plates are used for the meat, potatoe and vegetables. The plates are nickel silver with three divisions and fitted with nickel silver covers. These hot water plates are more or less satisfactory; we do not consider them ideal, but yet there seems to be nothing better on the market.

The food is sent to the different wards in bulk and served from the ward pantries, the trays being set and the tray dishes washed by the ward maids. Hot foods are sent in separate thermostat containers. This is fairly satisfactory though it is possible that these may be replaced, as they wear out, with insulated food carts. 3

1Editorial, Journal of American Dietetic Association, 9, 405 (1934).

${ }^{2}$ Philbrick, M. H. "Pioneering in Dietetics," Journal of American Dietetic Association, Al2; Chicago, IL, $19 \overline{36 .}$

${ }^{3}$ Barker, E., "What the Budget did for the Dietary Service of Flower Hospital," Modern Hospital 23: 160 (Hig) 1924 . 
As the dietitians influenced the operations of the foodservice system, the demands for new equipment to help to provide the services necessary, became greater. The dietitians wanted greater control over the diets and portion size. In order to help the patients to eat their meals, they realized that the food must be served at a palatable temperature. This places great importance on temperature maintenance systems and delivery systems.

Labor in most of the earliex hospitals was low paid or volunteer. Many hospitals were operated by religious orders, the military, or public government. The employees often worked for less because they felt they were doing charitable service. Labor unions developed in some of the hospitals across the united states. This influenced the establishment of labor saving equipment and safety standards.

The United States government legislated the minimum wage. The wage structures for hospital workers were at a lower scale than other industries. It was not until the early 1970's that the minimum wage went over $\$ 2.00$ per hour. Labor being so inexpensive, hospitals did not provide more labor saving equipment except where there was a shortage of labor. The changes in the labor market influenced the development of temperature maintenance and delivery systems. When labor was plentiful and inexpensive, fewer labor saving systems were implemented. Whereas a 
shortage of labor or higher wages caused the purchase of labor saving systems. Today we have plenty of labor and high wages which places more interest in labor saving systems.

Since World War II, many changes have been experienced in temperature systems and delivery systems. Refrigeration and heating systems have been developed from the technology learned during the war. The microwave oven concept was developed during this period but not implemented into hospital feeding until the 1960's.

The first heat maintenance systems used the insulated container concept. With the development of aluminum alloy pellets, hospitals were able to provide a constant heat source under each plate. Hot and cold carts were invented to take care of heating as well as cooling.

Tray assembly had been done on the floors but the dietitians felt that greater control would be obtained by centralizing this service. The debate as to which is the best way is still going on but with a third factor; that of a combination of centralized assembly and decentralized end heating.

Temperature maintenance and tray delivery systems have come a long way since their early beginnings. Thanks to Miss Nightingale and the dietitians who made people aware of the importance of food in the treatment of illness. This plus the economics of hospital food service has caused the development of hospital foodservice systems as we know them today. 


\section{CHAPTER I. PREREQUISITES}

In planning the food service system for a hospital, certain considerations must be made. Is the system for a new hospital which is being built or for an established one which is renovating its facilities? New hospitals are easier to plan because they are architecturally unlimited; on the other hand renovation of an existing structure is not as flexible. Money is the only limiting element.

The standard of food service must be determined in order to plan the system. This is done by the trustees of the hospital. The hospital can provide (1) nourishing food at the lowest possible cost, adequate, but without frills, or (2) food service equal to a good restaurant, that is, a limited selection of popular entrees, attractively served, and a few frills, or (3) meals and service equal to that found in luxury restaurants - those usually frequented only on gala occasions or by persons on expense accounts. I Once this has been determined then the design, layout, and construction can proceed.

After the standard of food service is determined then the type of service must be established. This can range

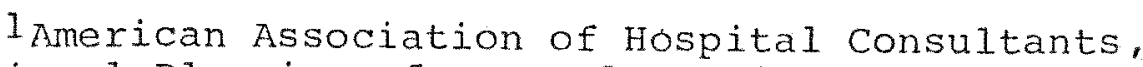
Functional Planning of General Hospitals, McGraw-Hill Book Company, New York, 1969. 
from total convenience to full raw production. The type and quantity of equipment varies directly with the type of service. Total raw production, seldom used today, requires different production equipment; whereas total convenience requires little production equipment but more storage space. The type of service influences tray assembly and temperature maintenance delivery systems, but there is no hard and fast rule which states that one particular type of service requires a certain tray assembly and temperature maintenance system. Therefore, this is a wide open area limited only by the inventiveness of those who are planning the assembly and temperature maintenance system.

There are two basic systems of hospital patient food service - centralized and decentralized. In the past the decentralized system was the procedure where the food was prepared in the main kitchen then placed in bulk in heated carts, usually one cart for each nursing unit. The cart was then sent to a floor pantry or kitchen where the food was portioned out on the plates, the tray was assembled, and then delivered to the patient. Today, with the growth of the microwave ovens and other types of ovens, the decentralized system incorporates the best of two worlds by assembling the trays in a central kitchen with the food in the chilled state. Then the 
trays are transported to the floor "galley" where they are held under refrigeration until it is time for the patient to be fed. ${ }^{2}$

When the patient is ready, the entree and other hot items are "end heated" for delivery to the patient. 3

In the centralized system the patients' meal is portioned out and his tray assembled in the main kitchen. In this simplest of systems, the assembled tray is delivered to the patient with the assistance of some heat maintenance equipment.

The debate as to which of the systems is best has been going on for years. The trend toward either one has been determined by the following factors:

1. Equipment available

2. New developments in heat maintenance

3. Cost of Iabor

4. Labor unions

5. Who does the final tray delivery, dietary or nursing service?

6. Control

7. Size of the facility

8. Expansion or remodeling

9. To improve patient satisfaction

10. Personal preference, either of the Food Director, Administration or Trustees

After all is considered, both sides (centralized and decentralized) have valid arguments. The effectiveness of either depends on how well they are instrumented, planned, and managed.

2pinkert, Michael s., The Ready Foods System for Health Care Facilities, Institutions/Volume Feeding Magazine, Chicago, IL, 1973.

3 Ibid., p. 120 . 
There are many types of tray assembly and heat maintenance systems. No matter what standard, type, and system is chosen, some basic considerations must be made. Some of the heat maintenance systems can only be used with disposables, namely Aladdin Cosmo and the Sweetheart cart, while others can only be used with reuseables, the 3-M system. This is an important consideration because of the market changes (as in 1974 when there was a plastic shortage and the high increase in the cost of disposables). The heat maintenance system should be flexible enough to adjust to such changes. Emergency situations also call for the same flexibility.

The size of the hospital also determines the heat maintenance and delivery and especially the tray assembly system. Some companies will not sell their systems to hospitals below 100 beds. Larger hospitals demand greater amounts of equipment, especially tray assembly lines where often two lines are needed to handle the high capacity. A tray assembly line can produce five trays per minute which means that 300 trays can be assembled in one hour. Smaller hospitals do not need this type of efficiency or the number of personnel needed to produce that many trays. Size also determines the time it takes for food to be transported from the central kitchen to the patient. This is critical in a centralized system.

A very important consideration to make in any decision on tray assembly and heat maintenance systems is sanitation. 
When dealing with sick people in the hospital this becomes even more important because of dangers of cross-infection or infection of a weak patient. Considering that food is being transported and delivered to all points in the hospital, every precaution must be taken to protect the patients from any contamination. Food temperatures, exposure, handling, and preparation must meet the most strict sanitary standards. In planning the tray assembly and heat maintenance system one should examine each piece of equipment and determine how it is to be utilized. The equipment should be easy to clean. Those items which maintain temperature should have specifications as to the length of time it will maintain the temperature. If there is preparation which is to be done away from the central kitchen, one should make sure that the conditions are sanitary and the preparation equipment is functional. The tray itself should adhere to sanitary standards in that all food should be covered and not exposed.

Maintenance is also very important. The equipment should be easy to maintain with little or no down time because a hospital must feed their meals 365 days a year with no break. Some systems require keeping backup equipment for just this purpose which is an additional expense. Parts and service should be readily available. Having to wait for long periods of time for parts to come half way across the country is very troublesome. Some companies 
have service contracts which are very good and useful. No matter how expensive or efficient a system may be, it must operate in order to do the job.

People eat with their eyes. This is especially true when a person is sick. They say, "I'm sick, nothing looks good to me." "The tray and its presentation should make the patient feel at ease with the food. The thing to avoid is anything which might turn the patient off because the food is important in the recovery of the patient. The tray assembly and the heat maintenance system should not be assembled so fast that they are done sloppily. The food should not be served in some abnormal manner just to protect the temperature. The tray should be attractive in order to enhance the food so the patient will enjoy eating the meal.

Financing and ownership of the tray assembly and heat maintenance systems are other points which need to be considered. There are two methods of financing and ownership; outright purchase and leasing. Both have their strong points. Leasing is available from many sources but some systems are available only on leasing terms. The lease agreement from those companies have no provisions for the hospital to purchase and own the equipment. Some companies state that they will provide the equipment needed to operate the food delivery system on a per meal pricing basis. This is actually a lease agreement. It is just a means of hiding the cost of the capital equipment. 
This being the case, when a per meal contract is being offered, the hospital should have a breakdown of capital equipment and the support supplies in order to appraise and evaluate the system against competition. These contracts also tie the hospital to a two to three year commitment. The thing to insist upon is a cancellation clause. Weighing these points, the hospital must make the decision whether the system is what they want and agree with the terms of the suppliers. If there is not a lease only offer, the hospital must determine whether to purchase outright or lease the equipment through private leasing firms.

The cost of the equipment is a major factor which will be reviewed in this paper. Some of the equipment on the market is quite expensive. The merits of the equipment lies in its efficiency and ability to control and lower costs of operation. Just how much can be saved is determined by the cost of labor and a comparison of the other equipment which could do the job for less.

In the final analysis, the tray assembly system and the heat maintenance system must fit the needs of the hospital, the personnel, and type of service which is expected, the economic needs, and patient satisfaction. 


\section{CHAPTER II \\ TEMPERATURE MAINTENANCE DELIVERY SYSTEMS}

Temperature maintenance, be it hot or cold is essential in every hospital food service system. The palatability and sanitation of food depends on temperature maintenance. The ideal system delivers all the courses of a meal at one time in an attractive presentation yet maintains every item at the ideal temperature until it is to be consumed.

Items that must be kept hot are the entree and vegetables, soups, beverages, hot cereals, and breads and rolls. The cold items are beverages, salads, desserts and cold entrees. Each are consumed at different times, so some provision must be made to maintain temperature until the particular food or beverage is consumed. The centralized and decentralized systems solve this problem in different ways.

The Centralized Food systems

Most centralized food systems use some type of heating or heating and cooling system to maintain the desired temperature from the time the food leaves the central kitchen until it is served to the patient. None of these systems is designed for reheating foods. Prompt delivery and minimum holding times are essential to any system if high quality food is to be served. The three methods of maintaing the 
temperature of food in the centralized system are (1) insulated containers and dishes, (2) pellet system and (3) heated and refrigerated tray trucks.

\section{Insulated Containers}

Insulated containers and dishes have been available for years. The thermos bottle was one of the first of this type. With the concept of double wall construction with a vacuum or insulating material between them, the development of plastic and stainless steel beverage servers, plastic and fiberglass plate holders, plastic and stainless steel bowls, plastic mugs, plastic tumblers, and even complete trays came to Eruition.

The next insulated containers were uniform in construction made out of styrofoam. This opened the door for the development of many new items. All of the styrofoam items are disposable. This started the increased usage of disposables because now there was something not only to maintain temperature but which was also disposable.

One of the first insulated containers for temperature maintenance was the Meal-Pak. 4 It was developed for the railroad for the feeding of passengers and was later adapted for hospital usage. The Meal-Pak was a stainless steel thermos container into which a heated three-compartment Pyrex dish was placed. (Exhibit \#1) It was hinged

${ }^{4}$ Dietary Products Division, American Hospital Supply Corp., Catalog, McGaw Park, IL, 1975. 


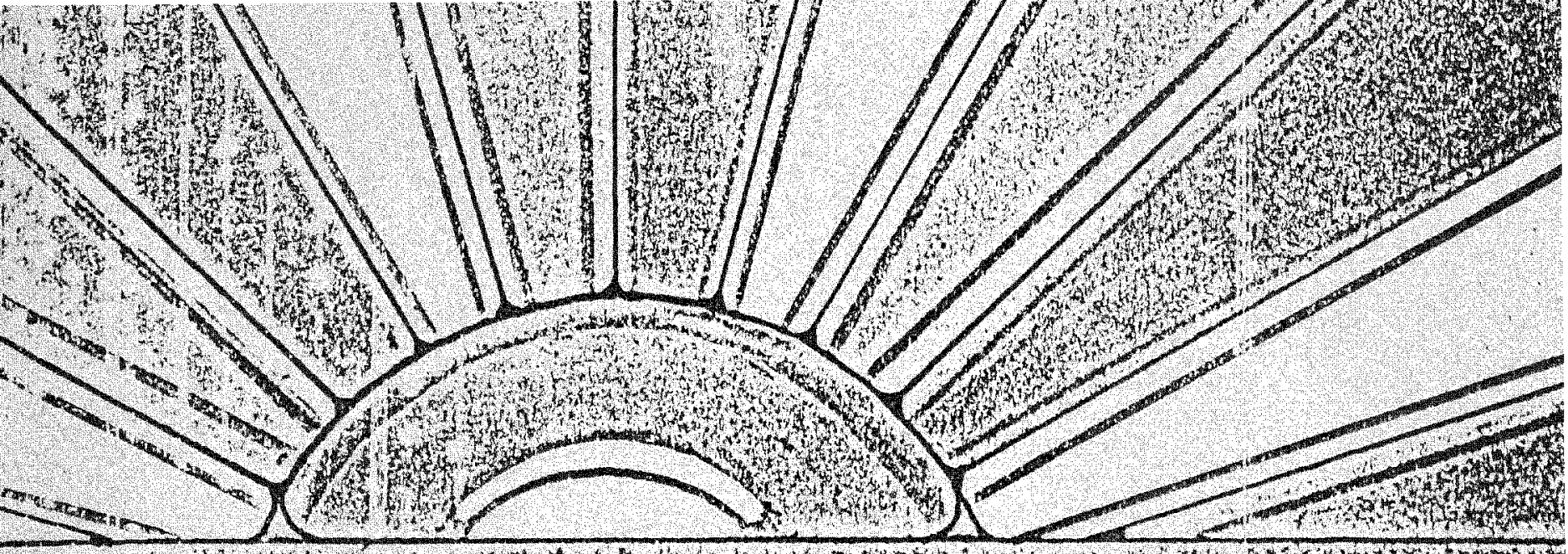


with a locking latch which sealed the container. Temperature retention was very good for up to 1 1/2 hours. Insulated bowls and beverage servers kept the other hot items warm. The system solved the problem of keeping food hot but it had its drawbacks. The unit was large and cumbersome. Only the Pyrex three-compartment plate would fit it. It was hard for the patient to open. The hinges and latches wore out due to extensive use. The smell of the food was trapped in the container and would sometimes bleed into the other items in the container. This would also cause the problem of the odor of the food to overwhelm the patient when he opened the container, especially with strong smelling items like fish and cabbage. Meal-pak was discontinued in 1971.

There are three insulated containers made to maintain the temperature of the entree. They are the Caddy-Templock, ${ }^{5}$ Dinex Insul-Server 6 and the Cambro Heat-Keeper. ${ }^{7}$ (Exhibits 2, 3, and 4) The Temp-lock and Insul-Server are made of plastic, while the Heat-Keeper is made of fiberglass. They are designed to hold a 9-inch plate, disposable or reusable, but preferably a reusable heated plate. The plates are supposed to be heated in a plate heater. This

${ }^{5}$ Caddy Corporation of American, Subsidiary of Rockaway Corporation, Catalog, Pittman, NJ, 1975.

${ }^{6}$ Dinex Products, Division King Seeley Thermos Co., Catalog, Norwich, CT, 1974.

${ }^{7}$ Cambro, Inc., Catalog, Huntington Beach, CA, 1975. 


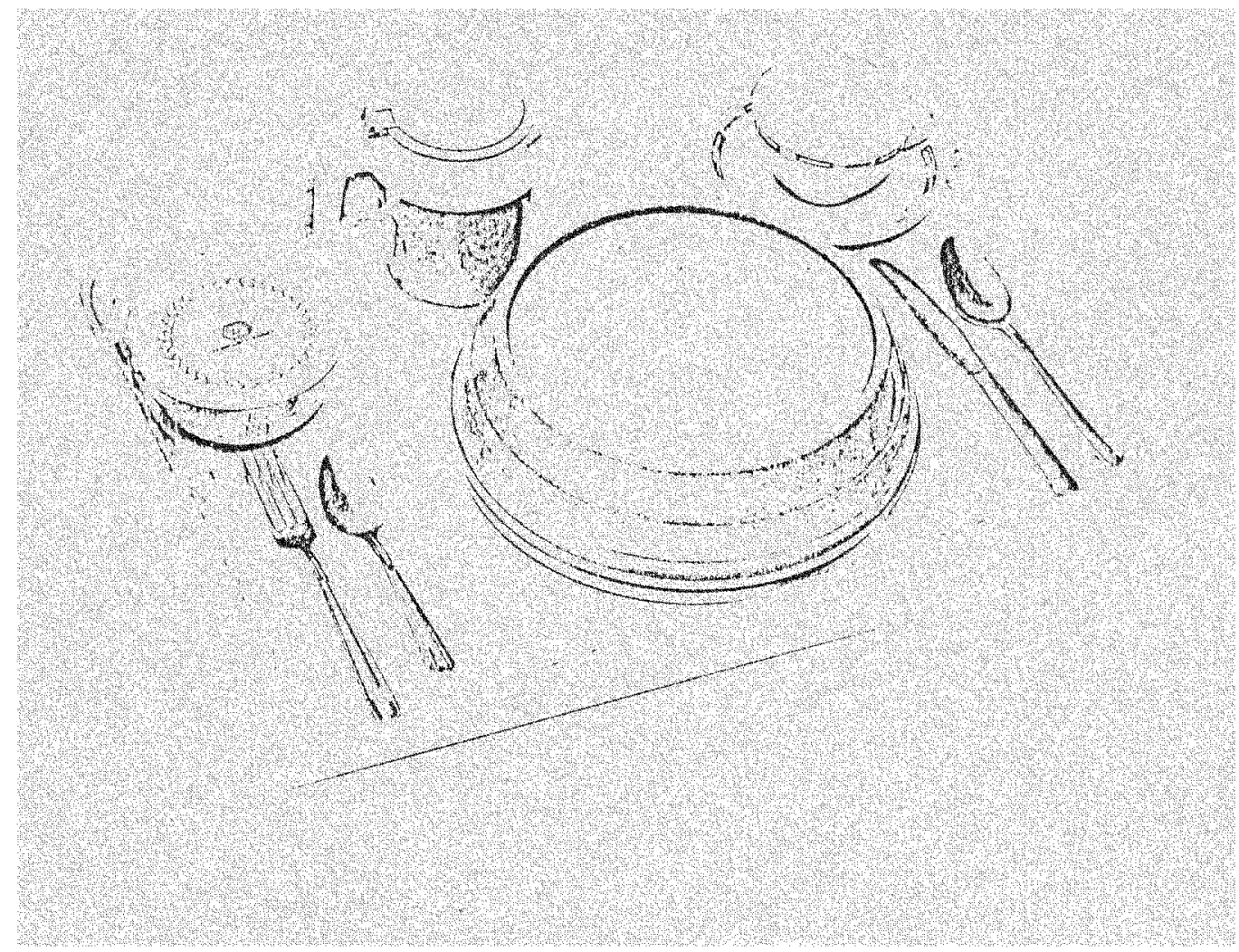

Exhibit 2 


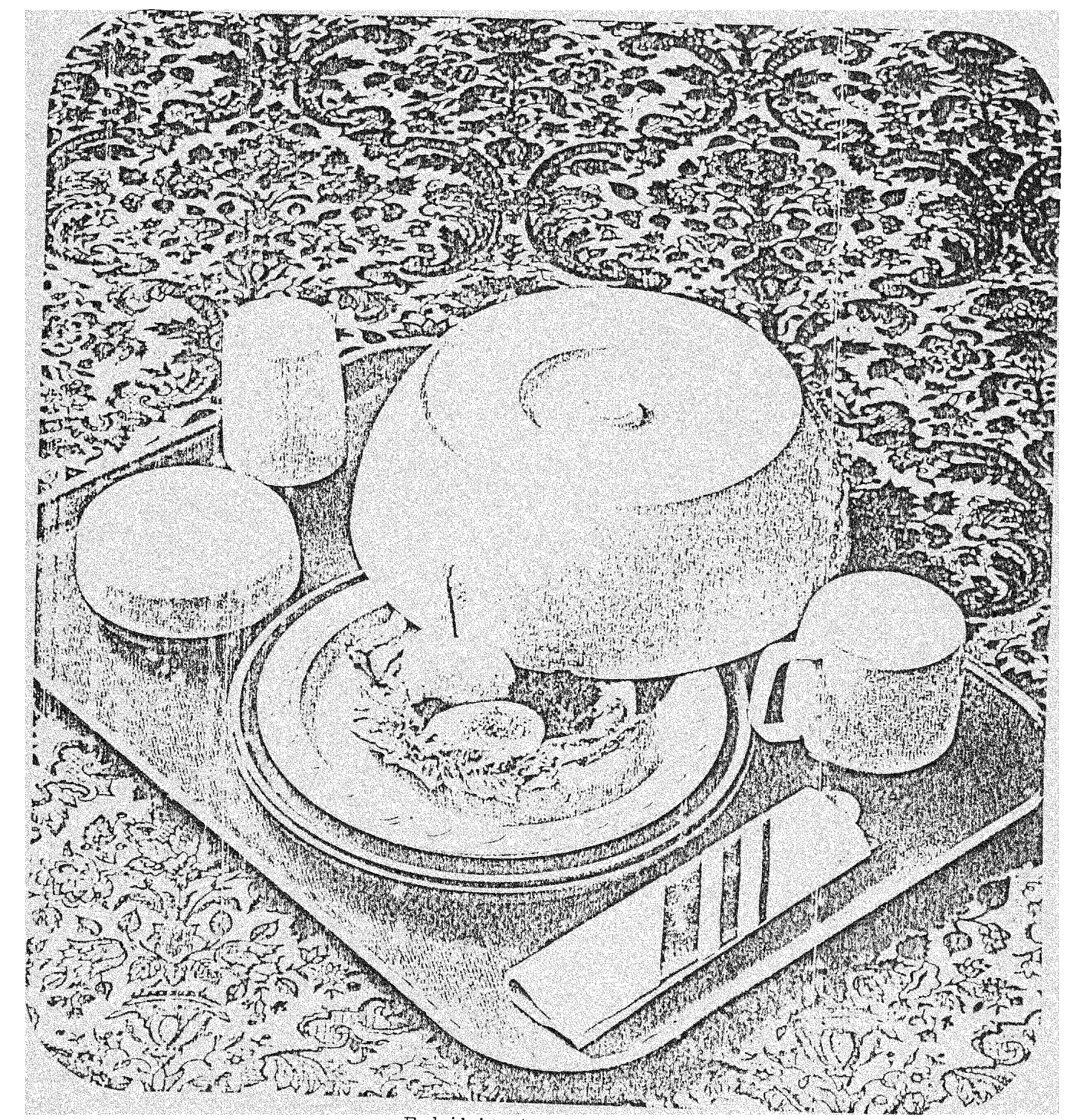

Exhibit \#3 
helps to maintain the temperature of the food. Cold plates can be cooled in a refrigerator before serving. The temperature of the food when plated is critical because the insulated container will only hold the food at that temperature. The holding time is 30 minutes. This makes these servers best suited for smaller hospitals or ones who have high speed delivery systems.

Advantages

Lightweight

Good heat retention if foods are hot/cold to start with

Minimal support equipment
Disadvantages

Short holding time

Not durable

Crack easily which causes

them to become unsanitary Hard to store

Cost:

Caddy Temp-Lock ${ }^{8}$

$\$ 14.40$

per set, base

and cover

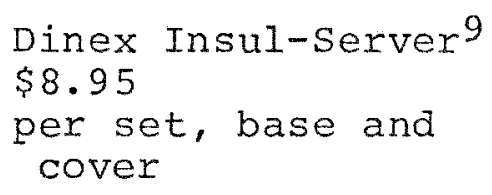

Cambro Heat 10 Keeper $\$ 12.50$ per set, base and cover

Heater Plate Dispensers (up to 72 plates per tube) (Exhibit 5) $\begin{array}{ll}\text { Shelley EDS2-H } & \$ 527.00^{11} \\ \text { Caddy Rh-S-21H } & \$ 1090.00^{12}\end{array}$

${ }^{8}$ Caddy Corporation of America, Subsidiary of Rockaway Corporation, Catalog, Pittman, NJ, 1975.

${ }^{9}$ Dietary Products Division, American Hospital Supply Corp., Product Information Manual, McGaw Park, IL, 1975.

10 Cambro, Inc., Catalog, Huntington Beach, CA, 1975.

${ }^{11}$ Shelley Manufacturing Co., A Division of Alco Food Service Equipment Co., Miami, FL, 1975.

12 Caddy Coxporation of America, Subsidiary of Rockaway Corporation, Catalog, Pittman, NJ, 1975. 


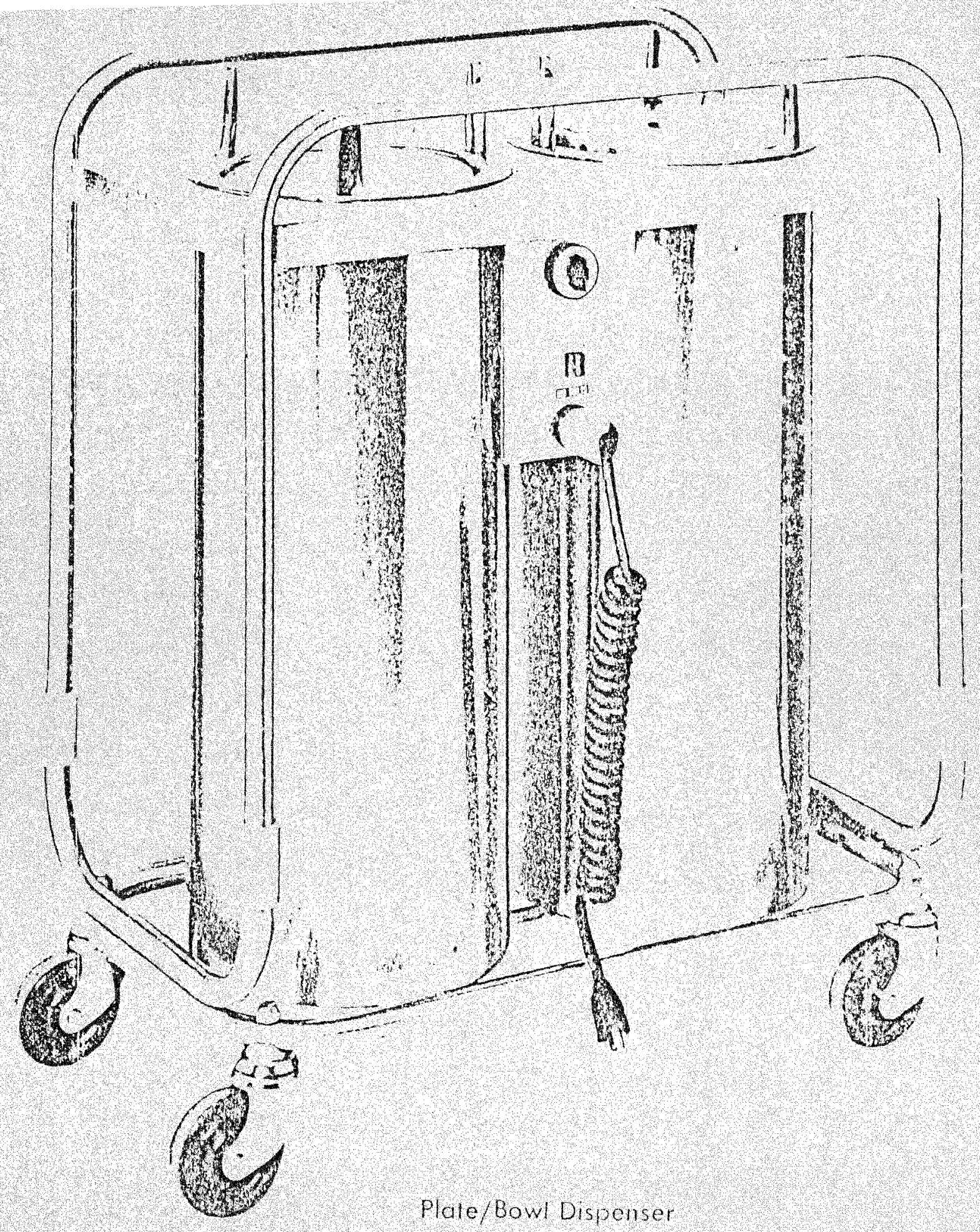

Exhibit $\$ 5$ 
Other companies which manufacture heated plate dispensers ${ }^{13}$ are:
Alco/U.S. Range, Inc.
Alto-Shaam, Inc.
Arista Metalcrafts Co.
Crescent Metal products,
Inc.
Curtis Equipment Corp.
Duke Manufacturing Co.
Food Equipment Corp.
Hatco Corp.
Hot Food Boxes, Inc.

Levelator Corp.

Merco Products, Inc.

Molitor, Inc.

Precision Metal Products, Inc.

Progressive Corp.

Seco Company, Inc.

Vulcan-Hart Corporation

Washington Equipment Co., Inc.

N. Wasserstron \& Sons, Inc.

Storage racks for the bases and covers are available. The racks hold 20 covers per shelf. One model, the caddy Model T-545, 100 capacity sells for $\$ 643.00 .14$ (Exhibit 6)

In order to keep the other items on the tray hot or cold, other insulated containers have been developed. This includes soup bowls, tumblers and mugs. The temperature maintenance statistics are attached. (Exhibit 7) These items use disposable lids to seal in the temperature and prevent spillage. The containers are made of either stainless steel or plastic. The plastic are less expensive and add color to the tray. For example:

Dinex Mug - 88c each ${ }^{15}$ (Exhibit 8)

Dinex Beverage Server - $\$ 2.00$ each (Exhibit 8)

Stainless Steel Beverage Server - \$5.25 each

(Exhibit 9)

Stainless Steel Bowl - $\$ 2.85$ each (Exhibit 10)

13 Foodservice Equipment Dealer, Product Directory, Cahners Publishing Co., Chicago, IL, 1974.

14 Caddy Corporation of America, Subsidiary of Rockaway corporation, Catalog, Pittman, NJ, 1975.

15Dinex Products, Division King Seeley Thermos Co., Catalog, Norwich, CT, 1974. 


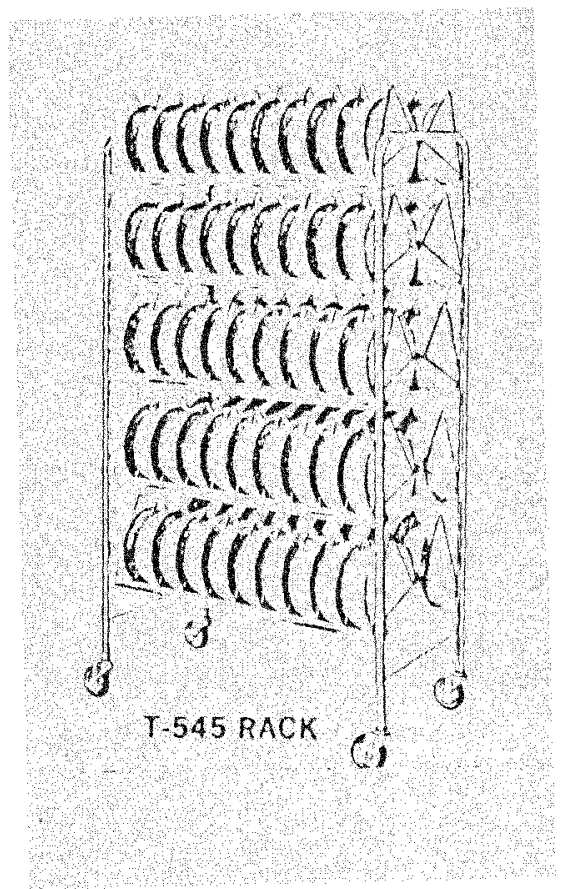

Exhibit \#6 
Tests conducted at 72 degrees Fahrenheit, room temperature, using boiling water as a test medium. Underlined figures indicate time at which heat retcrition crops below 140 degrees which is $A$. D. A. recommended minimum serving temperature. All figures shown are in degrees Fahrenheit.

CUP 8 Oz.

Without Lids

\begin{tabular}{|c|c|c|c|c|}
\hline Ceranic & China & DINLX & DINIX & Eyro Ceramic \\
\hline 125 & 125 & 125 & 125 & 140 \\
\hline 212 & 212 & 212 & 2.1.2 & 212 \\
\hline 180 & 202 & 195 & 198 & 192 \\
\hline--- & 146 & 170 & $\cdots$ & -- \\
\hline--- & 142 & 166 & $m$ & $\cdots$ \\
\hline 136 & 132 & 156 & 176 & 165 \\
\hline 129 & 120 & 147 & 171 & 151 \\
\hline 118 & 106 & 130 & 162 & 144 \\
\hline $\mathrm{X}$ & $x$ & $x$ & 151 & 124 \\
\hline$x$ & $x$ & $x$ & 141 & $\mathrm{x}$ \\
\hline$x$ & $\mathrm{X}$ & $x$ & $x$ & $x$ \\
\hline$x$ & $x$ & $x$ & $x$ & $x$ \\
\hline$X$ & $x$ & $x$ & $x$ & $x$ \\
\hline
\end{tabular}

* Cup \& Bowl Lids - Disposable F.D.A. Polystyrene. 


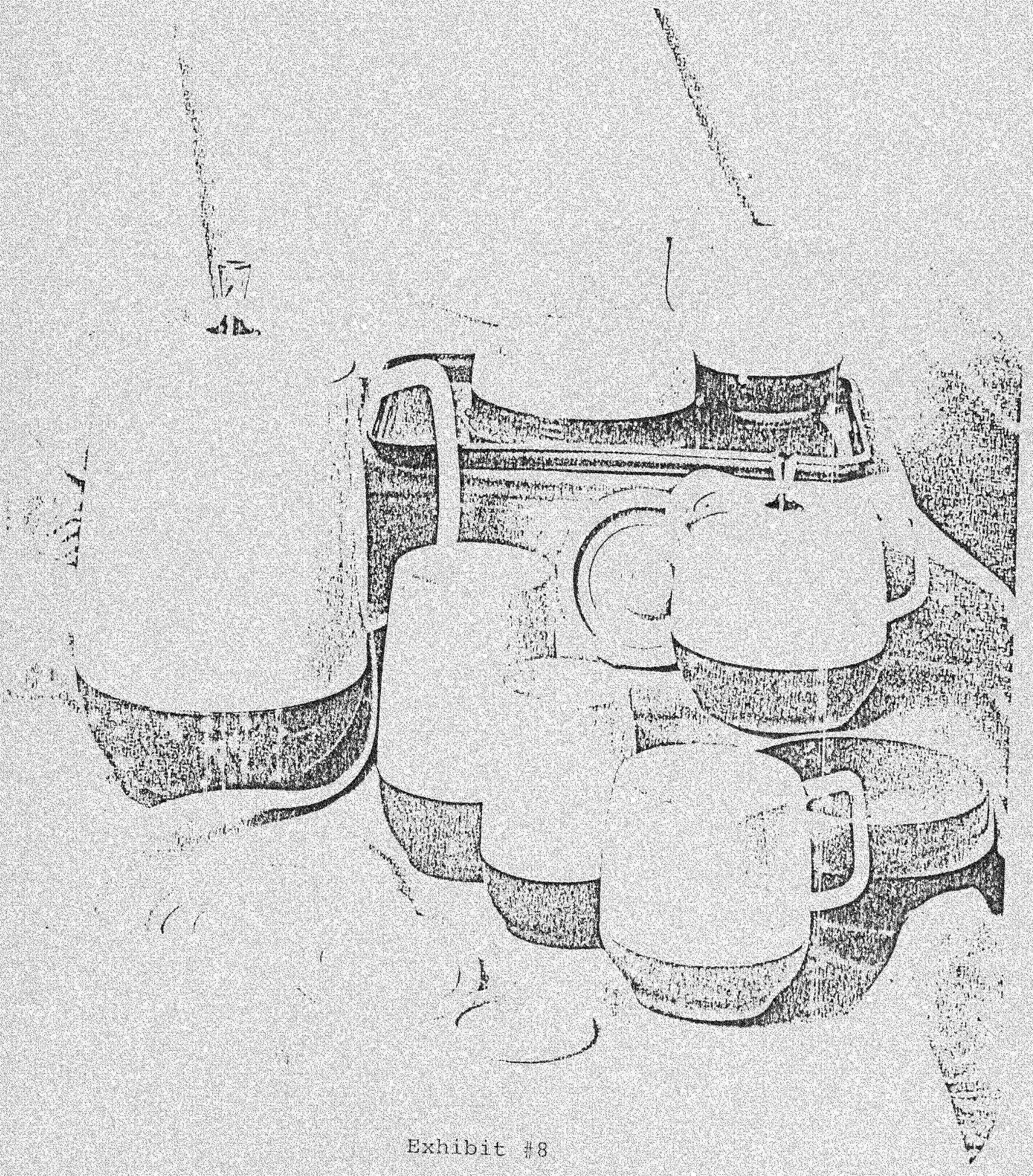



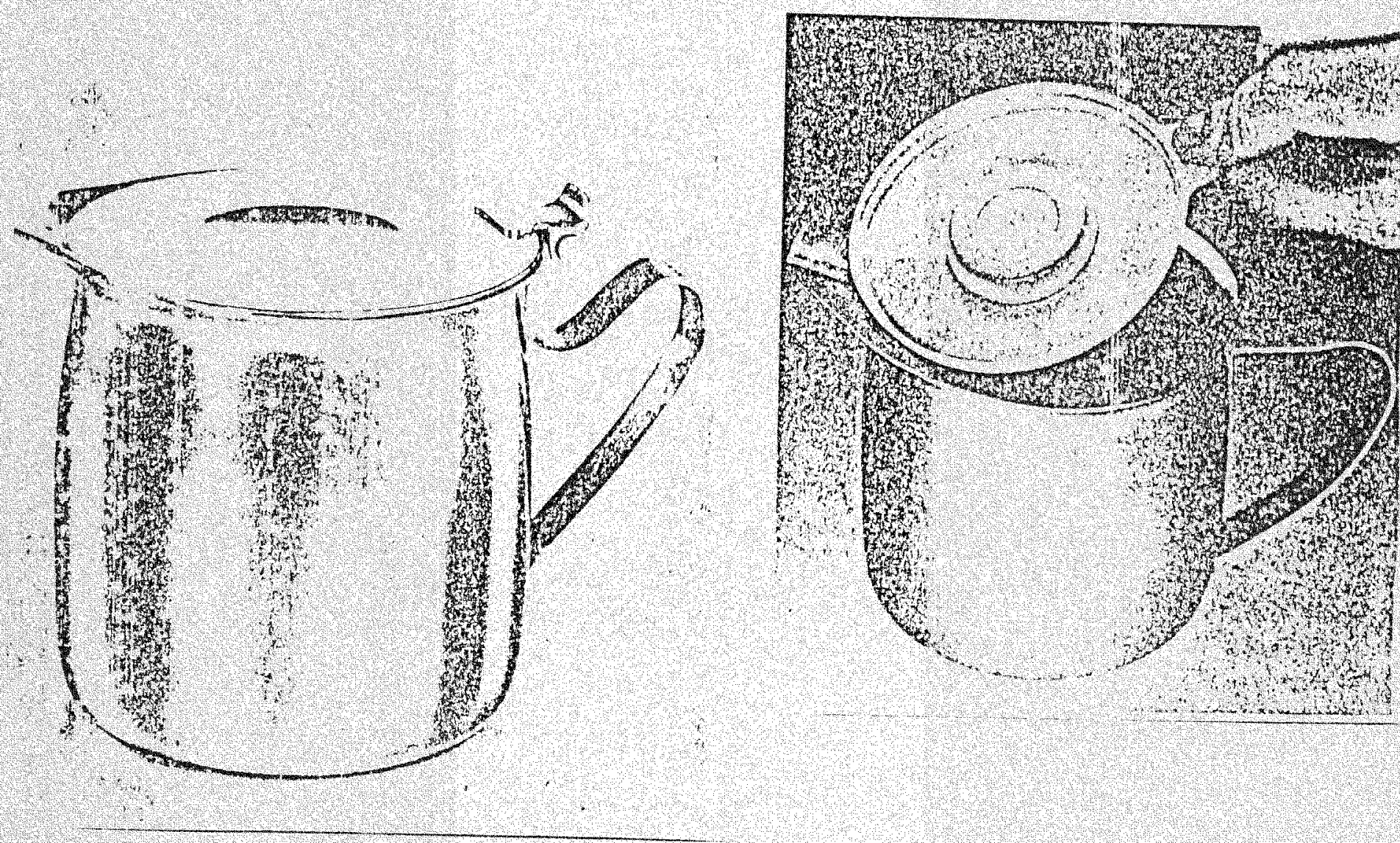

Exhibit \#9 


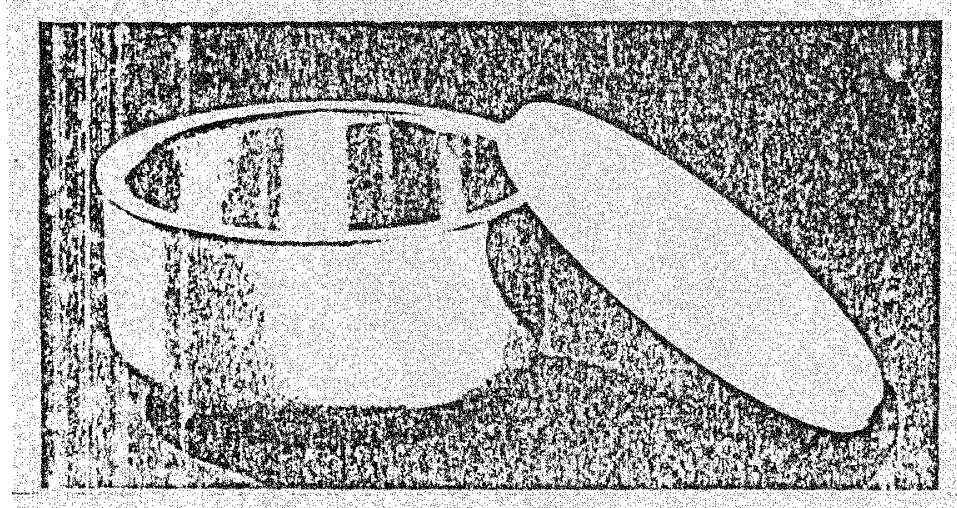

Exhibit \#10 
The mug, if used, replaces a cup and saucer and beverage server. Therefore, there is one item rather than three which means fewer items to crowd the tray, to handle, wash and buy. The insulated tumblers have not been used very much.

A typical system for a 200 bed hospital would be:

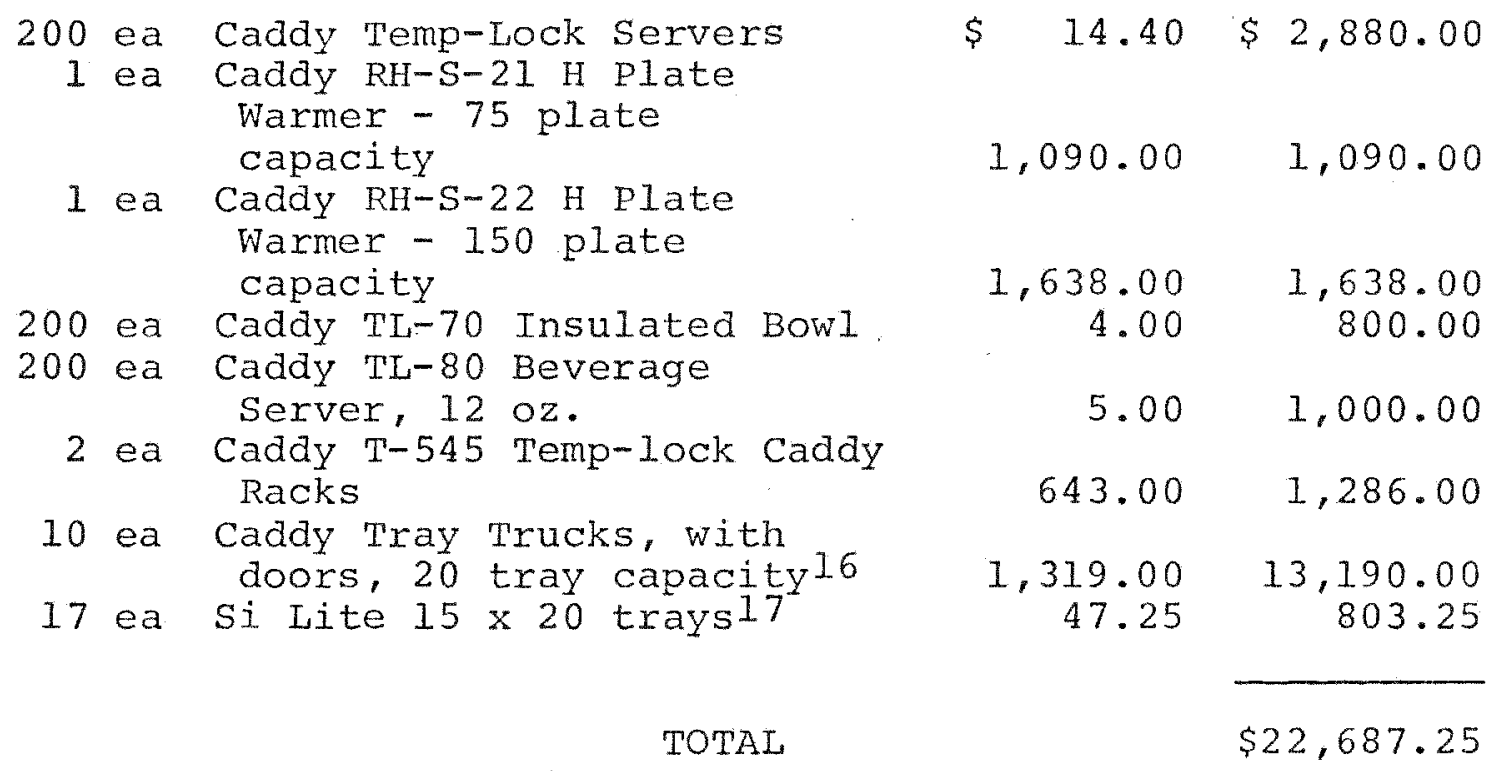

* China and silverware not included.

* Coffee cup and saucer will be needed.

This is an average of $\$ 113.43$ per bed.

${ }^{16}$ Caddy Corporation of America, Subsidiary of Rockaway Corporation, Catalog, Pittman, NJ, 1975.

17 Dietary Products Division, American Hospital Supply Corporation, Catalog, McGaw Park, IL, 1975. 
Alladin Synergetics Inc. carried the principle of the insulated container a step further. They developed a tray that was insulated (Exhibit 11). They market three basic configurations of trays. One small home delivered meals tray approximately 10" $x 12 "$; the cosmo series ( $I$ and II) which are small 12" $\mathrm{x} 15^{\prime \prime}$ trays with square compartments; and the Metro series ( $I$ and II) which are large 15" x 20" individually covered trays using, square and rectangular dishes with the option to go to a reuseable entree dish.18

Aladdin systems are predominately disposable and typically have problems with durability due to the overall construction of the tray. Water weight gain due to seam leakage leads to potential bacteria problems with each unit. The tray will hold meals at a proper temperature; hot foods hot, and cold foods cold, with no external heat or refrigeration required. The critical factor is the temperature of the food to start with. Some of the food does not fit under the insulated covers, namely the juice and milk. Problems derived from the initial trays caused the development of the Cosmo II and the Metro trays. The problem was that each tray was used as a cover for the tray below it. This left the food uncovered when the trays were being distributed to the patients. This caused temperature loss and sanitation problems.

18 Aladdin Synergetics, Inc, A subsidiary of Aladdin Industries, Inc., "From Kitchen to Patient Non-stop," Nashville, TN, 1975. 


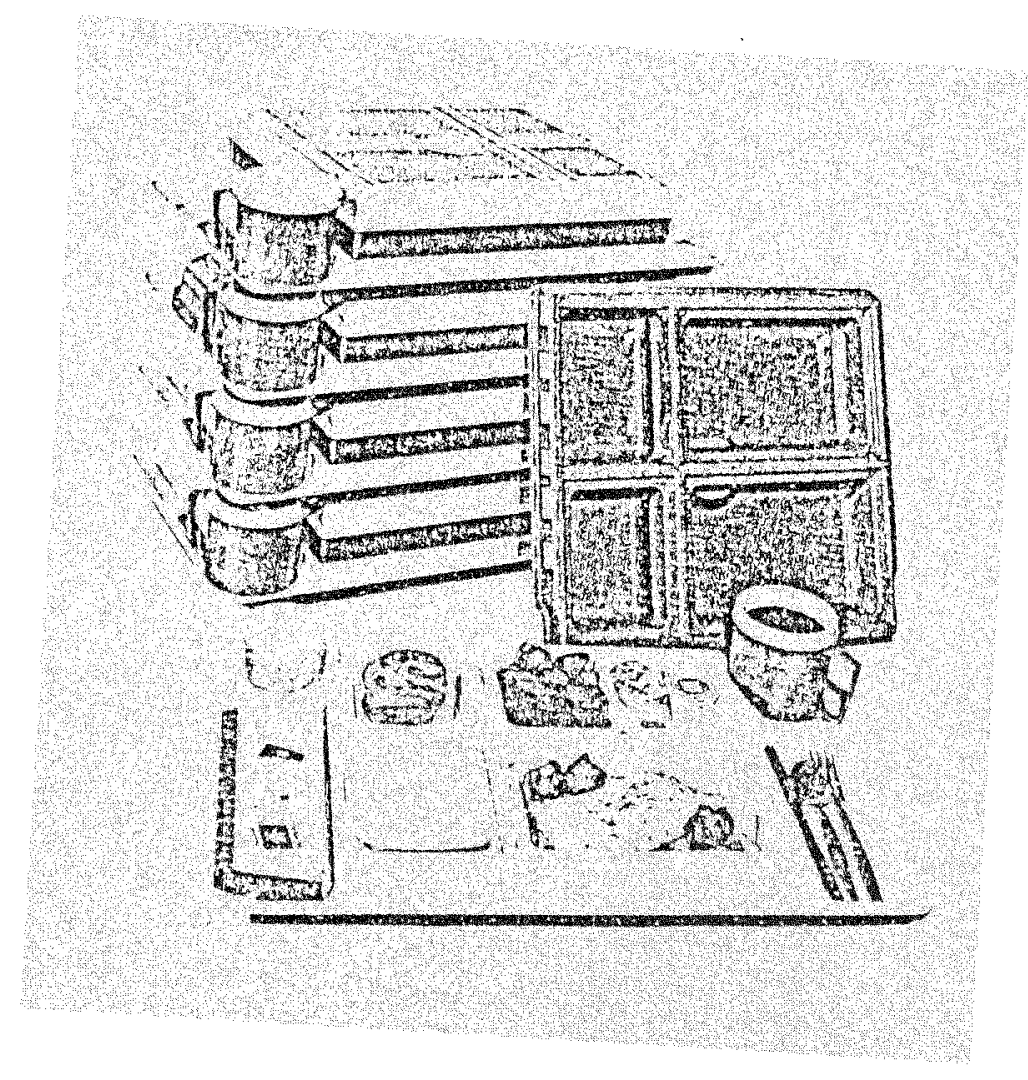

Exhibit \#II 
The trays are transported to the patient floors on a simple lightweight cart. They are easy to handle but some assembly is needed on the floors where the beverages are added.

The main thing that Aladdin did was to sell a disposable system without selling disposables. They sold the Aladdin system. They did this by changing the procedure of purchasing systems. Instead of selling the system outright, they sell the system on the per meal basis. The hospital is offered a contract for two or more years based upon the number of meals they serve. This charge included the equipment needed and the disposable components needed to make the system work. The hospital gets a monthly bill for a set number of meals. An example of this for a 200 bed hospital at $80 \%$ census would be:

$$
\begin{array}{r}
160 \\
\times \quad 3 \\
\hline 480
\end{array} \text { meals per day }
$$

$\frac{x 365}{175,200}$ meals per year

\section{5,200}

$$
\$ 22,075.20 \text { total cost per year }
$$

This equals $\$ 1,839.60$ per month. The $12.6 \%$ is broken down into two parts, the components and the equipment. The equipment cost of $6.8 \mathrm{f}$ is a fixed charge which will not change during the duration of the contract. The component 
charge of $5.8 \mathrm{f}$ is variable. 19 It fluctuates with the amounts used. The hospital is given an estimate of usage. If less than the estimated amount is used, a credit is given but if more is used an additional charge is made. The hospital must keep a month's supply of disposable components on hand. No local warehousing of products is available.

\section{Advantages}

Lightweight

Small

Good heat retention if foods are hot/cold to start with

Minimal support equipment

No capital investment
Disadvantages

Non-traditional square compartments

Not durable

Compartments are too small for many items

The system cannot be purchased outright

Others beside Aladdin have developed insulated trays. They are Thermo-Tray and Dietary Products. The Dietary Products tray was taken off the market during 1975. The Aladdin tray has been the most successful.

The insulated tray is a vexy good concept but it has had some difficulties adapting to the changing market. The insulated tray is mainly associated with the centralized system. The increasing cost of plastics have caused an increase of the price of the trays and components and have increased the scarcity of the product. In my opinion, the value per dollar spent is not very good.

19Aladdin Synergetics, Inc., A subsidiary of Aladdin Industries, Inc., Contract, Nashville, TN, 1975. 
With the advent of styrofoam came disposable insulated plastic containers. The heat retention of styrofoam is very good. Thermal conductance of styrofoam bowls, plates, and dishes is 1.5 degrees $F$. per minute as compared with 15 degrees $F$. per minute thermal conductance of paper plates. This is five times better insulation than aluminum. 20 The thickness of the styrofoam determines the retention qualities. Most of the styrofoam used in hospitals are stamped plates and dishes. They are rather thin and do not provide much heat retention. The covers are not made of styrofoam so there is a lot of heat loss through the cover. The next most used products are styrofoam cups and soup containers. They are very economical and good heat retainers. (Exhibit 12)

The disposable insulated plastic containers are used in many of the decentralized systems which use microwave ovens. The heat retention is not good enough to hold the food from a centralized kitchen unless it is put into a heat pellet container or hot and cold cart. Isolation is another area where these containers are used. Here, more expensive containers with better heat retention can be used. These have styrofoam covers. (Exhibit 13)

20Dietary Products Division, American Hospital Supply Corp. Product Information Manual, McGaw Park, IL, 1975. 


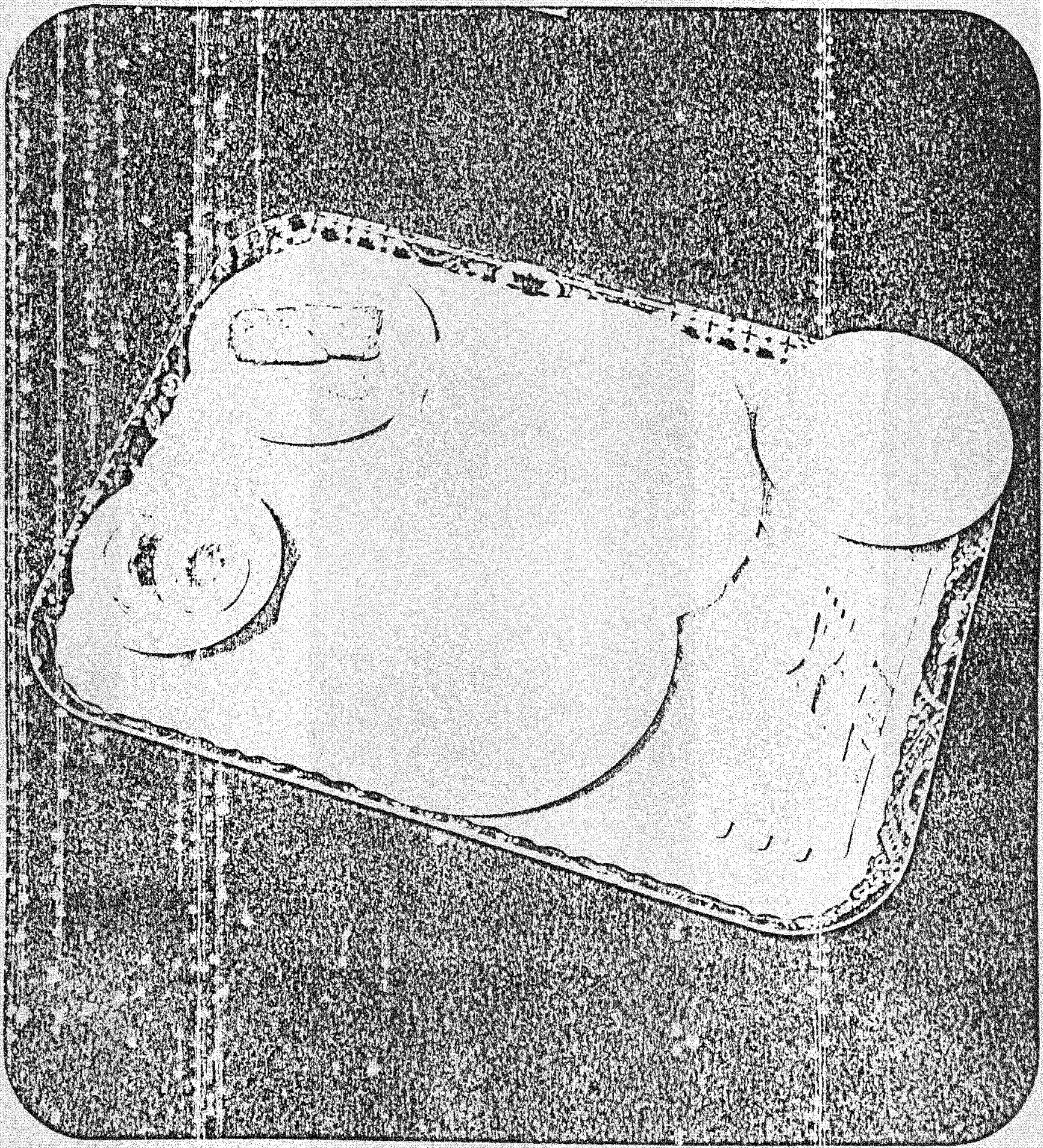

Exh1 b1t $\$ 12$ 

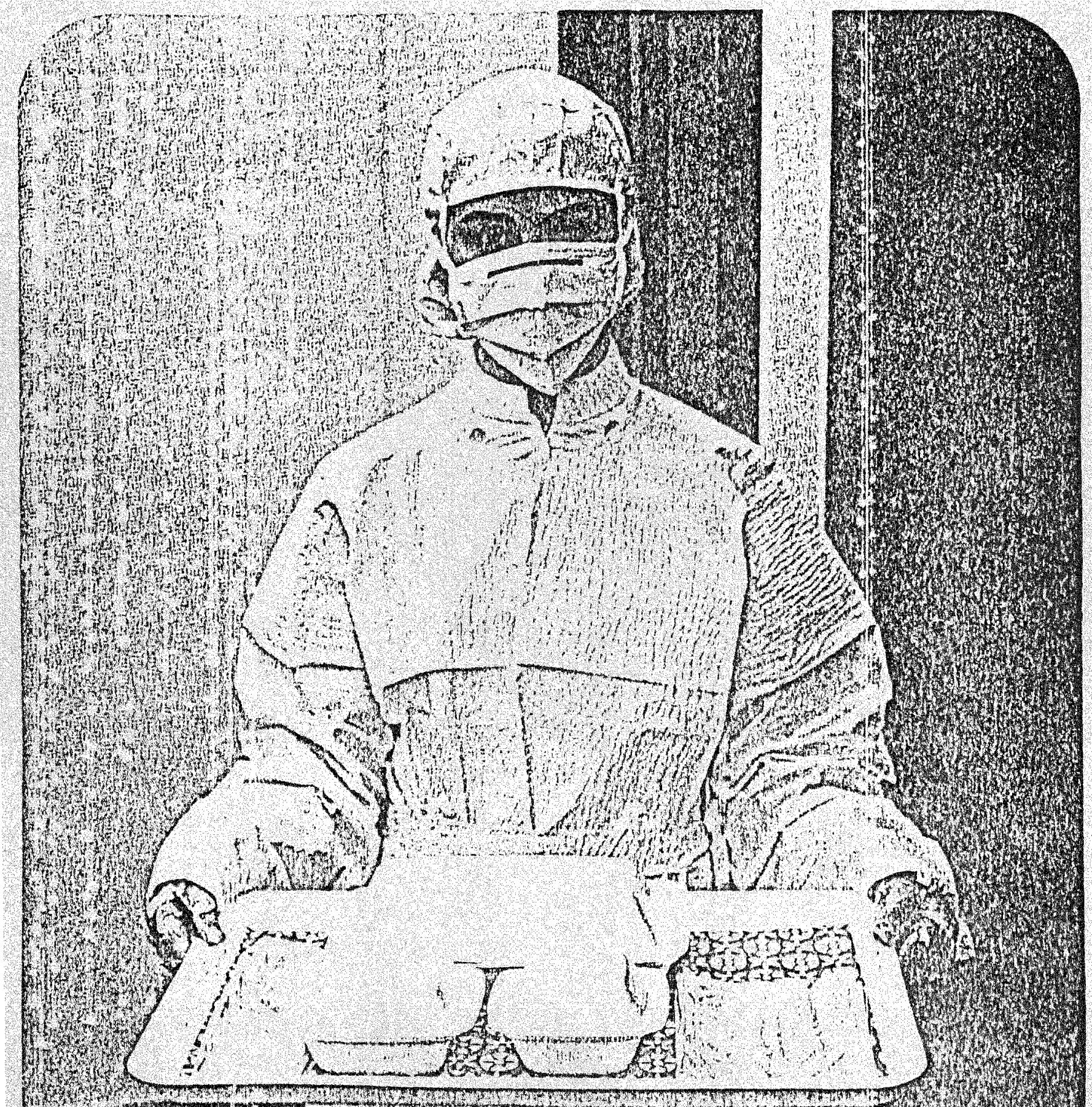

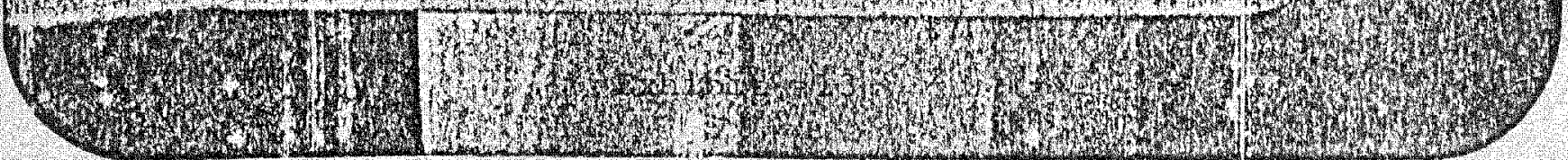


The major manufacturers of the styrofoam plastic containers are: 21

Amoco oil Company

Maryland Cup Company (Sweetheart)

Mobil oil Company

AIWF

Dart

Mars

\section{Advantages}

Lightweight

Disposable

Heat retention

Strong

Sanitation, less

cross infection

\section{Disadvantages}

Picnic look

Most do not retain

temperature for

prolonged periods

costly, if labor is not

saved

Not first class service

To evaluate the cost of these aisposable plastic containers to other systems would be unfair since most of the disposable plastic containers are used in coordination with other heat maintenance systems or for special types of service such as isolation. The disposable containers are usually sold on a per case basis. They vary in price as to the quality of the product. The quality is based on the thiskness, strength, color, and type of styrofoam used. The cost of a complete set of disposable plastic containers would be:

2lFood Service Marketing, EIP, Inc., Madison, WI, May, 1974 . 


$\begin{array}{ll}9 " \text { plate } & 4.3 \xi \\ \text { cover for above } & 6.7 \xi \\ 6 " \text { palte } & 2.0 \xi \\ 5 \text { oz, bowl } & 2.0 \xi \\ \text { lid for bowl } & 2.0 \xi \\ \text { l2 oz bowl } & 2.6 \xi \\ \text { lid for } 12 \text { oz. bowl } & \frac{2.6 \xi}{22.2 \xi 22}\end{array}$

Not all of the components are used on all trays. Liquid diets, and variations from the regular diet often use fewer components.

The complete isolation set-up with disposable flatware and tray cover costs 80 \& per tray. 23

\section{Pellet Systems}

The pellet, or hot-disk, system maintains heat for an adequate period of time. In this system, a flat disk is heated to an extremely high temperature. The disk is placed under the main dish and covered with a dome cover. The pellet acts as an effective source of heat in maintaining the temperature of hot entrees.

The pellet systems have been used mainly with the centralized systems. They have made tray assembly and control much better because each tray is made up complete in the central kitchen with no assembly or preparation on the floors.

The major problem of the pelletized system is keeping the other items that do not fit under the main entree cover

22 Dietary Products Division, American Hospital Supply Corp., Catalog, McGaw Park, IL, 1975.

23 Ibia. 
hot and the cold foods cold. This is accomplished by using insulated containers to support the system. The cold foods are kept refrigerated until they are put on the tray. The first pellet system was the "doughnut" pellet system. (Exhibit 14) The small doughnut pellet is made of a special alloy metal pellet which is heated to approximately 450 degrees $F$. The pellet is dispensed in the insulated plateholder. Then the plate is placed on top of the pellet.

Since the pellet is small and requires high temperatures for heating, heat distribution is not even, certain plates will melt over the high temperature and the food continues to cook. To remedy these problems, a larger aluminum alloy pellet was invented. It is heated to 225 degrees $F$. in an infra-heater or a heated self-leveling dispenser. (Exhibit 15)

In order to solve the problems of high loss of the pellets and three pieces to wash, a unitized pellet was developed. This fused the pellet between two stainless steel plates in the base. There is a layer of fiberglass at the bottom to prevent the heat from radiating to the tray but rather upward toward the food. The new unitjzed pellet is warmed to 225 degrees $F$. in self-leveling convection heaters. Since the temperature is lower than the doughnut pellet, all types of plates can be used in the unitized pellet. (Exhibit 16) 


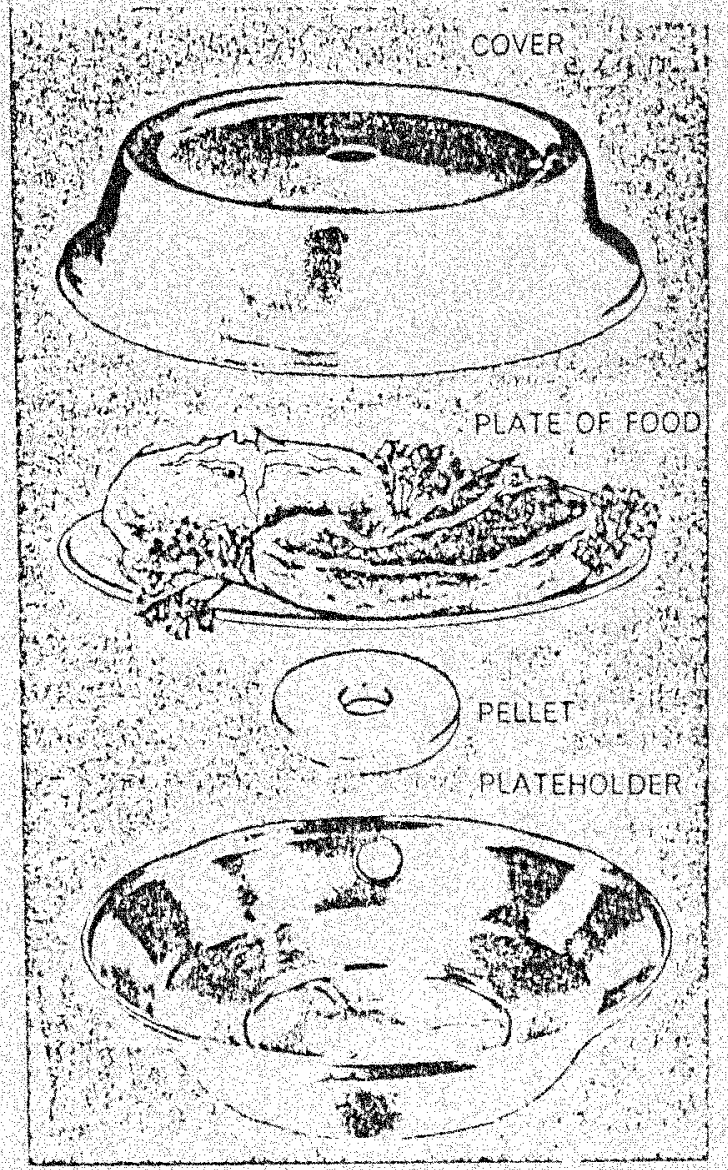

Exhibit \#14 


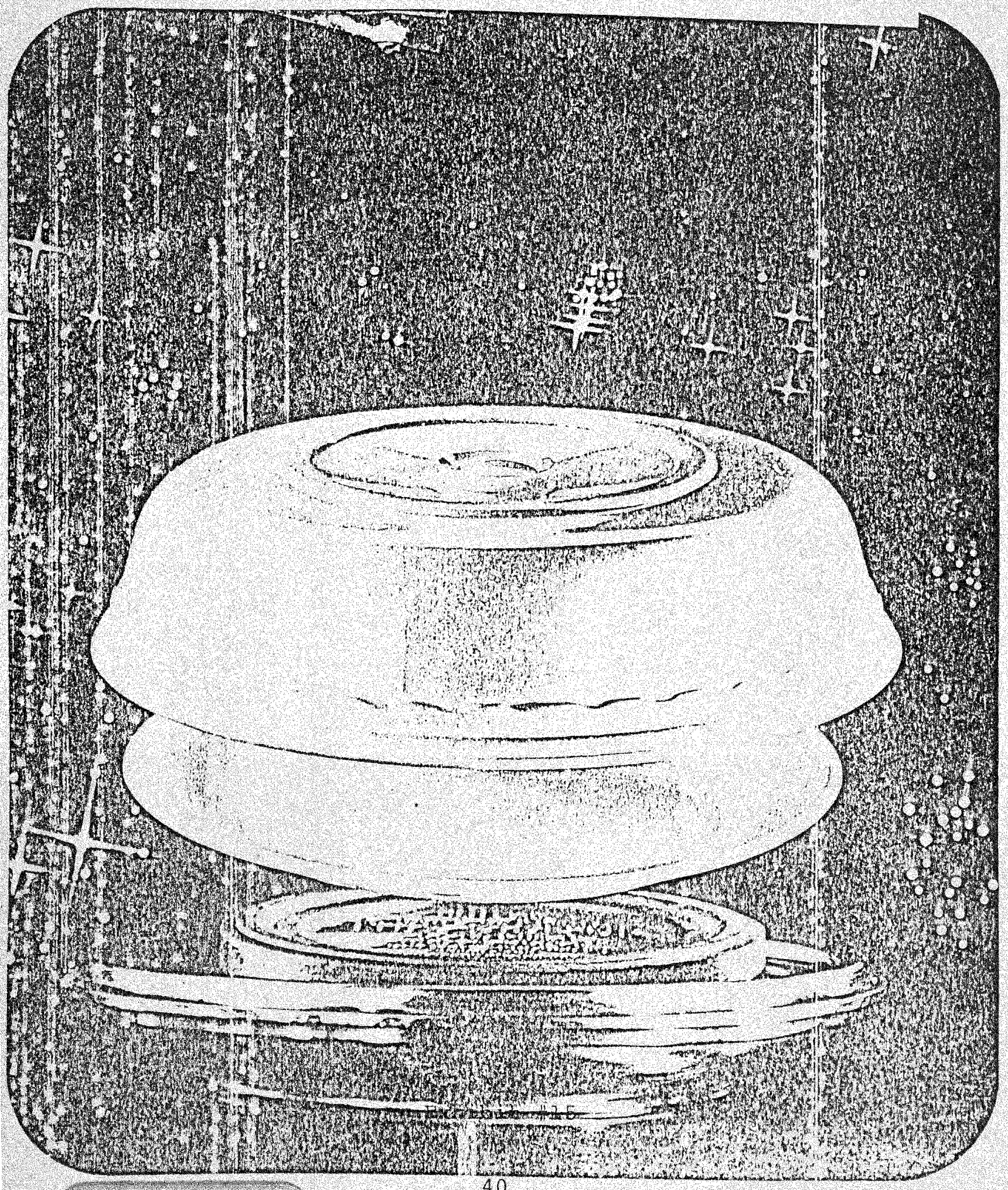




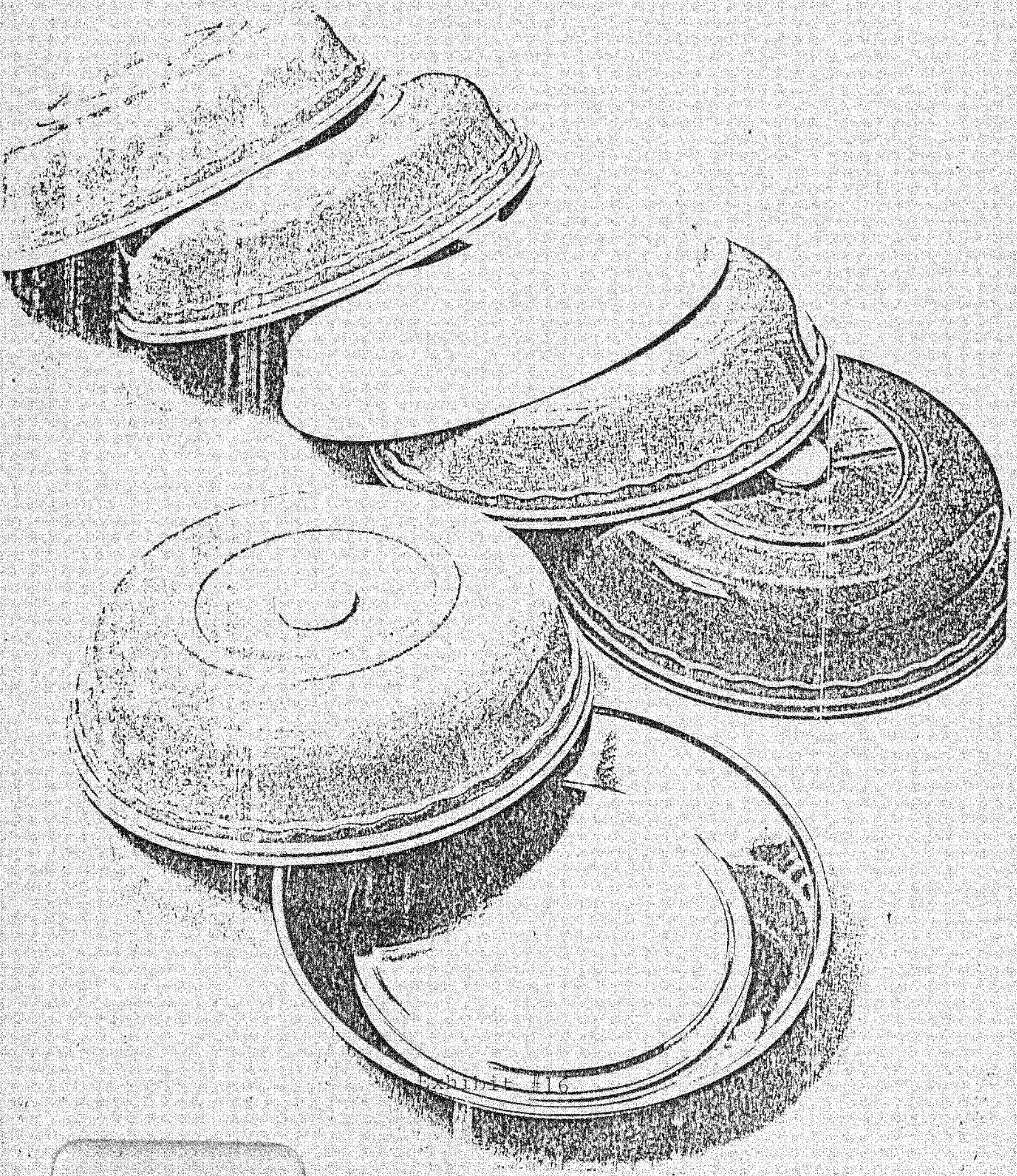


The heat retention of the unitized pellet is very good. (Exhibit 17) The food is not cooked but kept in a warm state. Food remains hot after it is removed from the tray cart. The heat source stays under the patients' food so the food stays hot until they are ready to eat. This is useful since all the courses are served at the same time, the patient will eat his appetizer and soup before he starts on his entree. If the heat retainer was not there, he would have cold food before his meal was completed. The pellet system is economical in that it does not require the large amounts of electricity, the amount of help and the large capital investment that other systems require.

After the trays are assembled, the trays are transported to the floors in unheated carts. (Exhibit 18) These carts are lightweight and easy to handle. They come in different sizes and different capacities. The size of the cart is based upon the number of trays which have to be served in each nursing unit. The tray slides into the cart should be on 4 inch centers so all the items can stand up on the tray. The smaller, lighter, greater the capacity and more maneuverable the cart, the better it is. The following companies manufacture carts:

Also/Shelley Mfg. Co. AMF Inc., Food Alto-Shaam, Inc. Ard Mfg. Co., Div. Brucken Co., Inc. Arista Metal Crafts Co. Arizona Booth and Fixture Mfg. Corp.
Atlas Metal Industries Haralson Metals Hot Food Boxes, Inc. Insinger Machine Co. Institutional Maintenance $\mathrm{Co}$. JCN Wire Industries Ltd.

Ladder Escape Co. of America Rack Division 
Bloomfield Industries, Inc. Brunor, Inc.

Bucksco

Caddy Corp. of America

Carter-Hoffman Corp.

Colson

Crescent Metal prods., Inc.

Crimsco, Inc.

Curtis Equipment Corp.

Dallas Sheet Metal Works, Inc. Duke Mfg. Co.

Eastern Steel Rack Co.

Dyna International

Ecko Products, Inc.

Epco-Div. of Std. Int'l

The Fabricators, Div of

Dallas Sheet Metal Works, Inc. Food Equipment Corp

Food Warming Equipment Co. Foremost Industries, Inc.

Foster Refrigeration Corp.

Frantz Industries

Raburn Products, Inc.

R.H.I. Products, Inc.

Seco Company, Inc.

Serv-O-Lift Corp.

Southern Equipment Co.

Star Metal Corp.

Sterno, Inc.

Technibilt corp.

Terris Consolidated Ind. Theodore Equipment Corp.

Tyson Metal Products Corp.
Lakeside Mfg. Inc.

Legion Utensils Co., Inc.

Levelator Corp

Lincoln Food Service Systems

Losli, Inc.

Market Forge Co.

Maxwell-Phillip, Div of

Sterno, Inc.

Metropolitan Wire Goods Corp.

Missouri Equipment Co.

Mobile Master Div., Stainless and Aluminum Specialty Co.

Modern Plastics Div.

Molitor, Inc.

National Equipment Corp.

Normandie Metal Fab., Inc.

Oneida Silversmiths

Original Metal Mfg. Co.

Pase Equipment $\mathrm{Co}$., Inc.

Polar ware Co.

Precision Metal Prod., Inc.

Union Steel Products Co.,

Div. of Eagle-Picher Ind.,

Inc.

United Service Equipment Co. The Vollrath Co.

Washington Equipment Co., Inc. N. Wasserstrom and Sons, Inc. Wear-Ever Food Service Equip. Co.

Wilder Mfg. Co., Inc.

Wyott Corp. 24

24 Foodservice Equipment Dealer, product Directory, Cahners Publishing Co., Chicago, IL, 1974. 


\begin{tabular}{|c|c|c|c|c|c|}
\hline \multicolumn{2}{|c|}{ Test: Temperature } & \multicolumn{2}{|c|}{ Specification: Verbal } & \multicolumn{2}{|c|}{ Project No: 70287} \\
\hline \multicolumn{2}{|c|}{ Conditioning: $200^{\circ}$} & \multicolumn{2}{|c|}{ Date: $6-18-70$} & \multicolumn{2}{|c|}{ Tested By: B.A.A. } \\
\hline \multicolumn{6}{|c|}{ Manufacturer: Dietary Division, AHSC } \\
\hline \multicolumn{4}{|c|}{ Material: Hospital Food Warmer } & \multicolumn{2}{|c|}{ Lab. Sup. Check: I: McL. } \\
\hline \multicolumn{6}{|c|}{$\begin{array}{l}\text { Remarks: Plate Used - "Melitta" "g" } \\
\text { "Food" - Bordens Whinoed Potatoos }\end{array}$} \\
\hline Time & $\begin{array}{l}\text { Heater Temp. } \\
\text { (One Hour } \\
\text { Saluration) }\end{array}$ & $\begin{array}{l}\text { Food } \\
\text { Temp }\end{array}$ & $\begin{array}{l}\text { Base } \\
\text { Duter } \\
\text { Euge }\end{array}$ & $\begin{array}{l}\text { Base } \\
\text { Muner } \\
\text { Evige }\end{array}$ & $\begin{array}{c}\text { Base } \\
\text { lmor Surface } \\
\text { (Center) }\end{array}$ \\
\hline $9: 15$ AM & $200^{\circ}$ & $148^{\circ}$ & $142^{2}$ & $159^{\circ}$ & $199^{\prime \prime}$ \\
\hline $9: 20 \mathrm{AM}$ & $200^{\circ}$ & $148^{2}$ & $122^{\prime}$ & 141 & $180^{\circ}$ \\
\hline $9: 25 \mathrm{AM}$ & $200^{\circ}$ & $148^{\circ}$ & $114:$ & 132 & $170^{\circ}$ \\
\hline $9: 30 \mathrm{AM}$ & 200 & 146 & $110^{\circ}$ & 126 & 161 \\
\hline $9: 35$ AM & 200 & $145^{\circ}$ & $108^{\circ}$ & 123 & 156 \\
\hline $9: 40 \mathrm{AM}$ & 200 & 142 & 105 & 118 & 148 \\
\hline $9: 46$ AM & 200 & $141^{\prime \prime}$ & 103 & 114 & 143 \\
\hline $9: 50 \mathrm{AM}$ & $200^{\prime}$ & 138 & 101 & 111 & $137^{\circ}$ \\
\hline 9.55 AM & $200^{\circ}$ & $133^{3}$ & $99^{\circ}$ & 109 & 132 \\
\hline $10: 00 \mathrm{AM}$ & 200 & $130^{\circ}$ & 98 & 107 & 128 \\
\hline
\end{tabular}




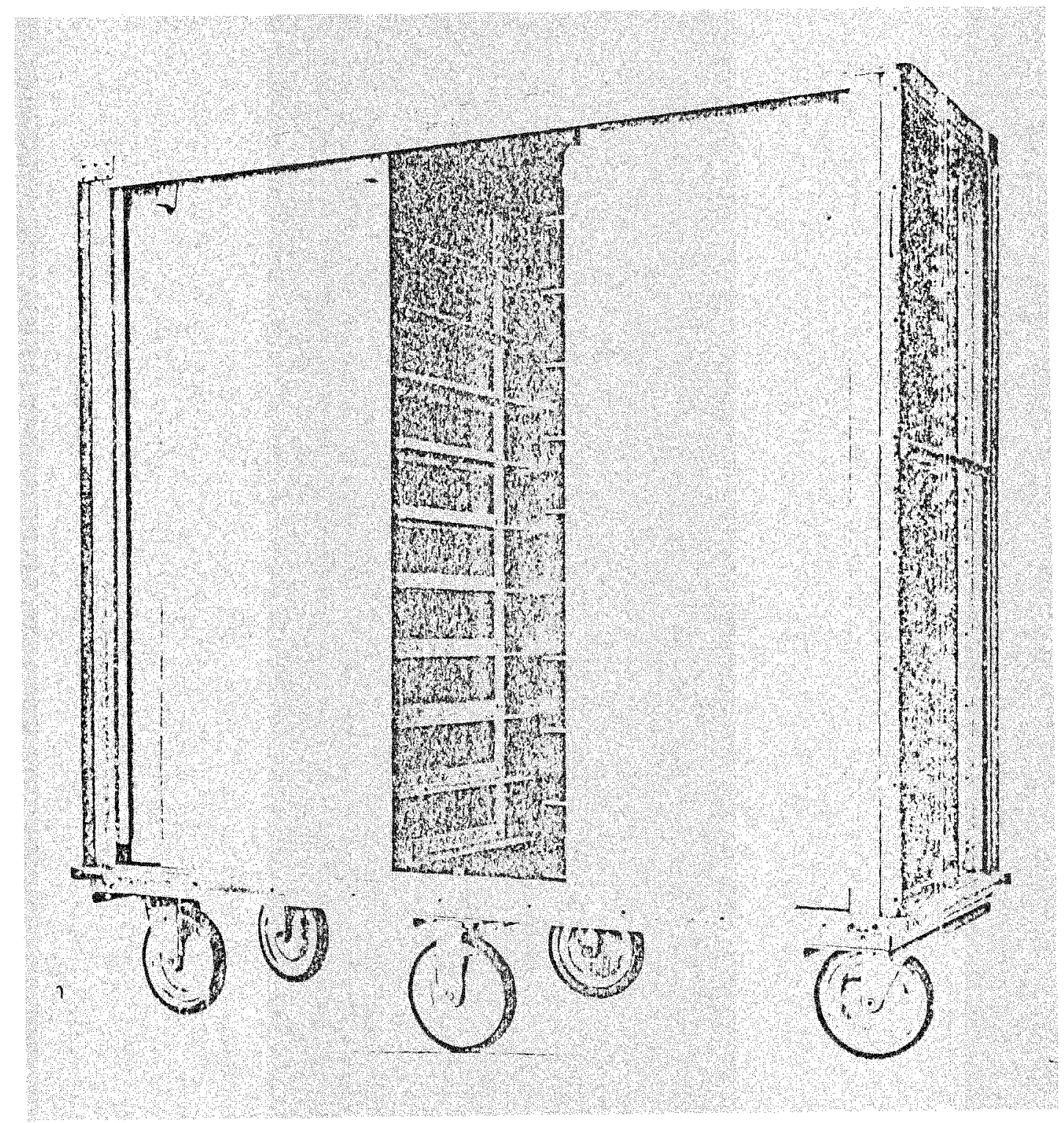

Exhibit \#18 
Cost for a 200 bed hospital:

Dri-Heat (Dri-Heat Division, Franklin Products Corp.) (Dri-Heat Franklin, 1975)

200 Dri-Heat Plate complete $w /$ pellet $\$ 27.30 \$ 5,500.00$

1 \#509 Dri-Heat Pellet Heater

90 capacity

1 H512 Dri-Heat Pellet Heater,

120 capacity.

200 Dinex 8 oz. bowls

200 Dinex 8 oz. mugs

1 Shelley EDSI-H Plate Dispenser

72 capacity

I Shelley EDS2-H Plate Dispenser

144 capacity

10 20-TCS Dri-Heat Masterline Tray cart

17dz Si Lite $15 \times 20$ Trays

(Dietary Products, Catalog, 1975)

$1,630.00 \quad 1,630.00$

$1,795.00 \quad 1,795.00$

$.80 \quad 160.00$

$.88 \quad 176.00$

$340.00 \quad 340.00$

$527.00 \quad 527.00$

$\begin{array}{rr}1,410.00 & 14,100.00 \\ 47.25 & 803.25\end{array}$

TOTAL

$\overline{\$ 25,031.25}$

China and silverware not included.

This is an average of $\$ 125.00$ per bed.

Hot-Pak (Unitized Pellet, Dietary Products)

200 Hot-Pak Bases

200 Domes Covers

2 d-Line Hot-Pak Unitized Base

Heater dispenser, convection

style

1 Shelley EDS1-H Plate Dispenser

72 capacity

1 Shelley FDS2-H Plate Dispenser 144 capacity

200 Dinex $8 \mathrm{oz}$. bowls

200 Dinex 8 oz. mugs

10 Shelley HDC-20 Tray Delivery

Carts

$17 \mathrm{dz}$ Si Lite $15 \times 20$ Trays

(Dietary Products Catalog, 1975)

China and silverware not included This is an average of $\$ 107.78$ per bed. 
In the latter part of 1973, Dietary Products Division American Hospital supply corporation introduced a square unitized pellet system called the 1216 system. (Exhibit 19) The 1216 system used the same basic heat retention system as the round unitized system but used a $7^{\prime \prime}$ square container and plate. The name 1216 was derived from the size of the tray which was used, a 12.x 16 low profile tray. The system was designed to replicate the airline type tray. The use of the smaller tray and the $7^{\prime \prime}$ square unitized pellet enabled the use of smaller carts with greater capacity. These carts (Exhibit 20) hold 28 trays, 2 per shelf. This reduces the number of carts that are needed and makes the cart easy to handle even by the female help. The cost of the cart made of stainless steel is $\$ 825.00$. This reduces the cost of the system for a 200 bed hospital by $\$ 7,225.00$ (compared to the Shelley HDC-20 carts). This same principle was used to redesign the round unitized system in 1975 to the Elegent Dining System. (Exhibit 21) The $12 \times 16$ tray was too smal1 to be used with a round unitized base which many of the customers wanted. A 12 x 22 tray was designed with a slightly enlarged cart. These carts sold for $\$ 975.00$ each. Recently some hospitals have started to use the unitized pellets in their galleys where they microwave their food. The reason for this is to keep the entree hot until the patient is ready to eat his entree and also to save labor. Five to ten trays can be done and held with the unitized pellet rather than running each tray to the patient 


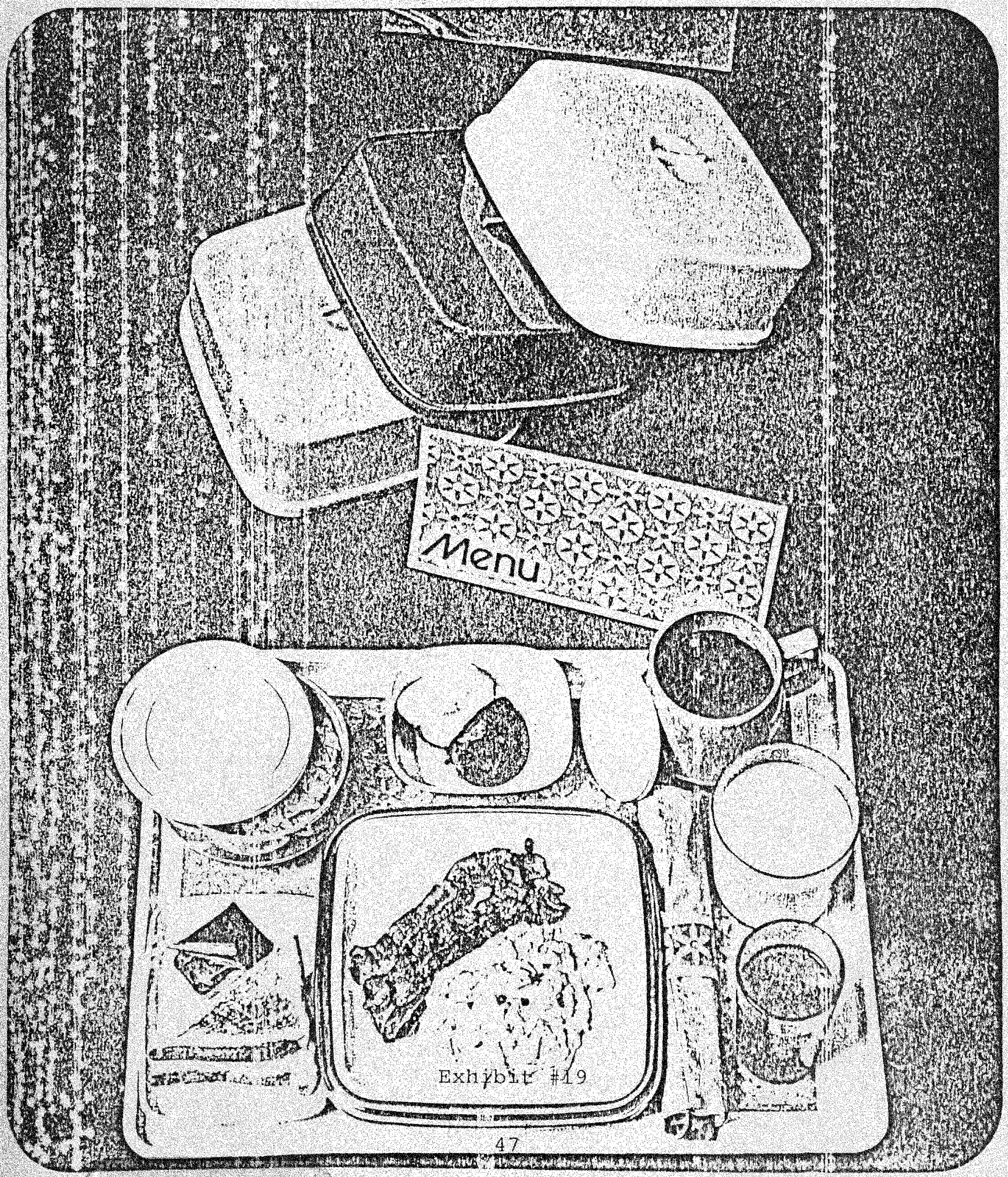




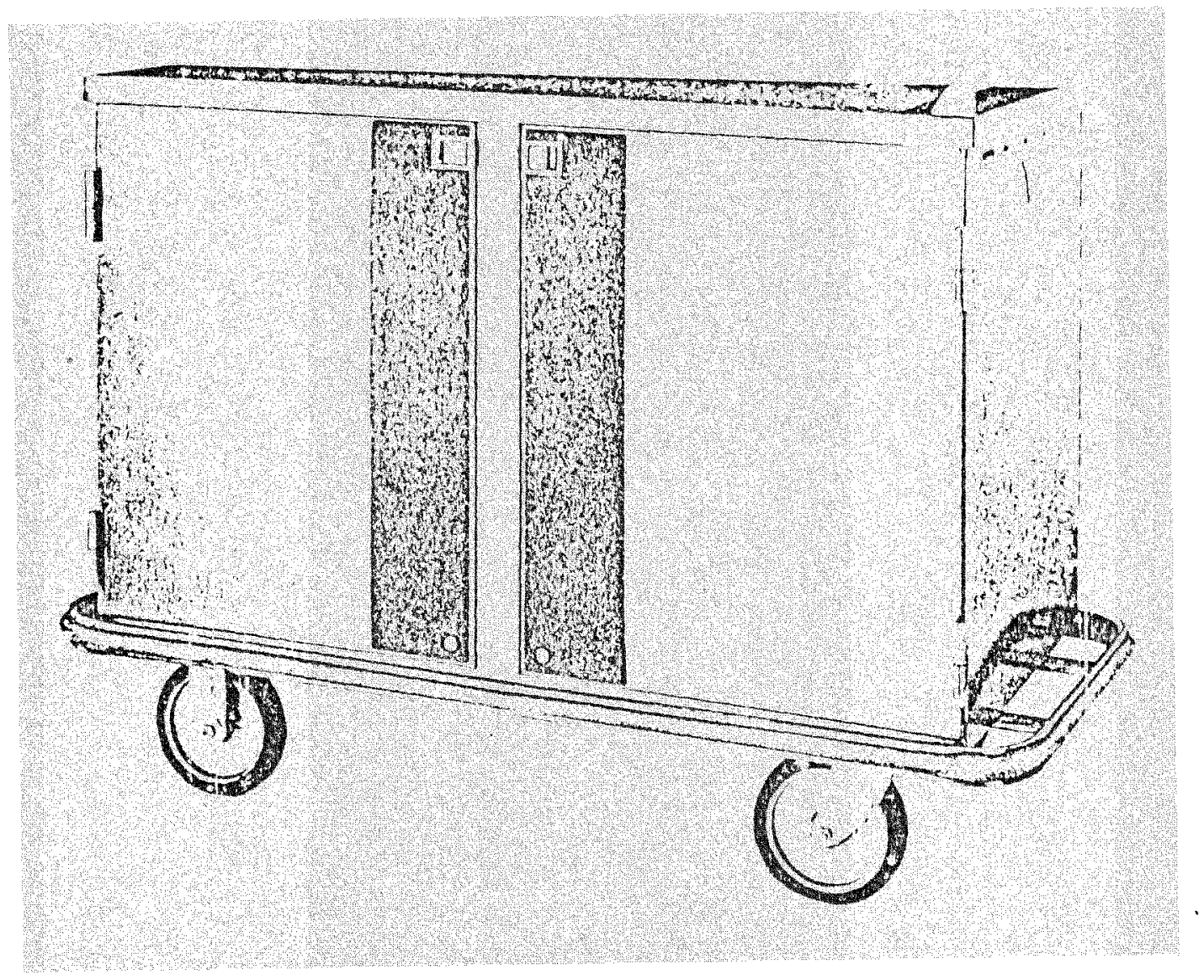

Exhibit \#20 


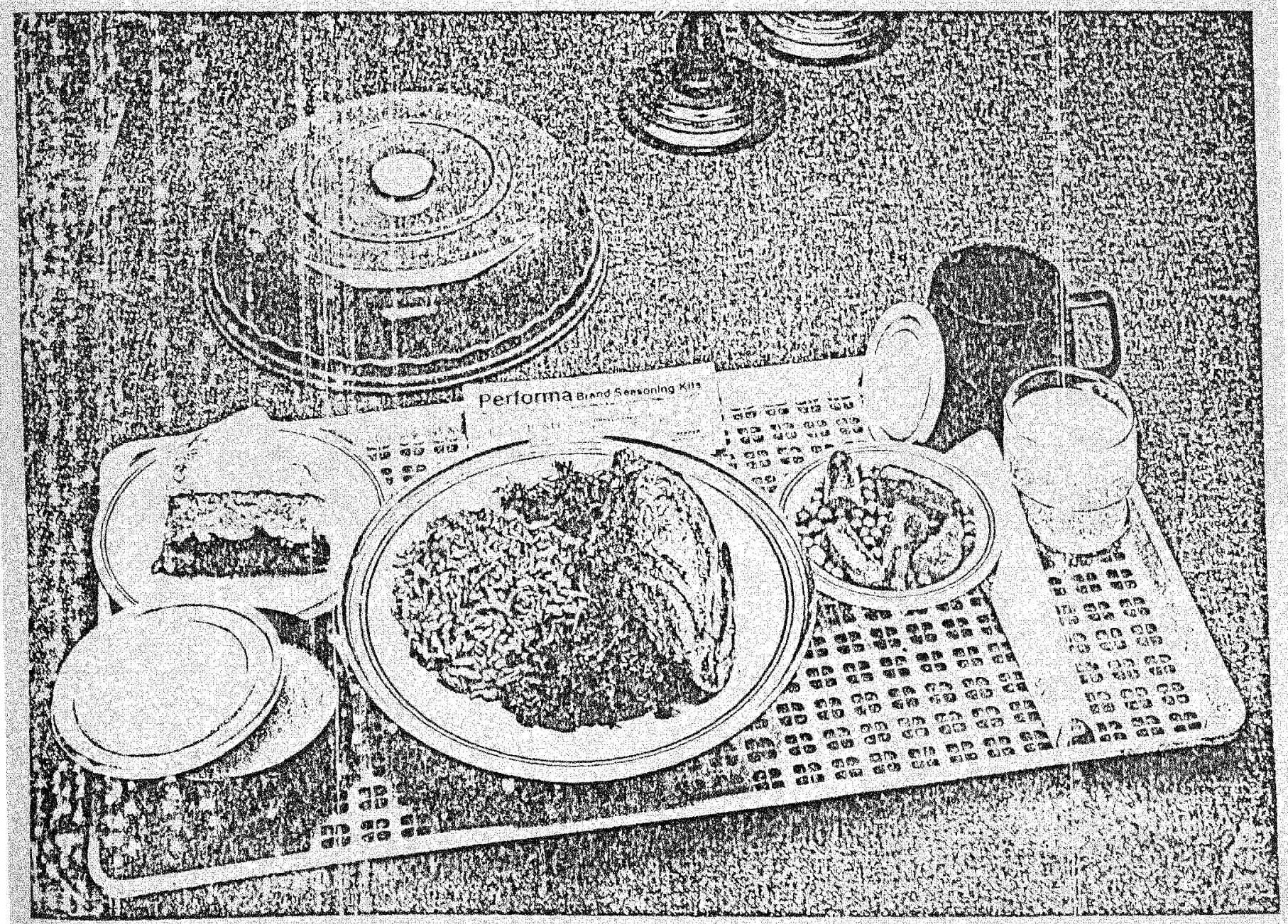

Exhibit \#21 
as it comes from the microwave. Some people say that this is a duplication of system and is not needed, but where it has been used the satisfaction of the management and patients is very high.

The manufacturers of the "doughnut" pellet are:

Dri-Heat Division, Franklin Products Corp. Savory, Alco Standard

The manufacturers of the unitized pellet system are:

Caddy Corp. of America

Dietary Products, Division, American

Hospital Supply Corp.

Brand Ware

United Service Equipment Company, Inc.

Advantages

Heat retention

Economical

Heat under entree at

all times

Flexible, usable with

china or disposable

Labor saving, fewer

people to deliver trays

Hot and Cold Carts

When confronted with the problem of temperature maintenance for hospital feeding, someone must have said, "Wouldn't it be nice to have an oven and a refrigerator outside of each patient's room to keep the food at the proper temperature?" Well, several companies did just that. Carts have been manufactured with heating sections and refrigerated sections. They are on wheels which make them mobile. These carts became popular with the growth of the decentralized system. The trays are made up in a central 
kitchen and loaded onto the carts which have been plugged in before the serving time to warm and cool the respective compartments. After the cart is loaded, it is transported to the floor where they are plugged in again. The food can be maintained at the proper temperature for up to two hours. The first hot and cold cart had two sections, a hot side and a cold side. The cold side held a $15 \times 20$ patient tray with all the items that had to be kept cold plus the tray top items, silverware, napkin, tray cover and glassware. Another tray was put into the hot side with all of the hot items. (Exhibit 22) When the cart got to the floor, the diet aides had to match the hot items to the cold items. Sometimes there were errors made; to prevent this, a split tray was implemented. The whole tray was assembled in the central kitchen. The hot food was placed on one side of the tray and the cold items on the other side. The cart was built up to accept the complete tray. A gasket divided the tray and prevented the heat from being transfered from either side. (Exhibit 23) These gaskets would wear out after a year's usage which caused the hot and cold sides to fight each other and become very ineffective.

\section{Advantages}

Long term storage of food on the floors Good if the carts are plugged in and the doors remain closed separation of hot and cold items
Disadvantages

Heavy carts - weigh over 400 1bs. possible reassembly on floors High storage, preheating and cooling space requirements Expensive

Mediocre thermal qualities High maintenance costs 


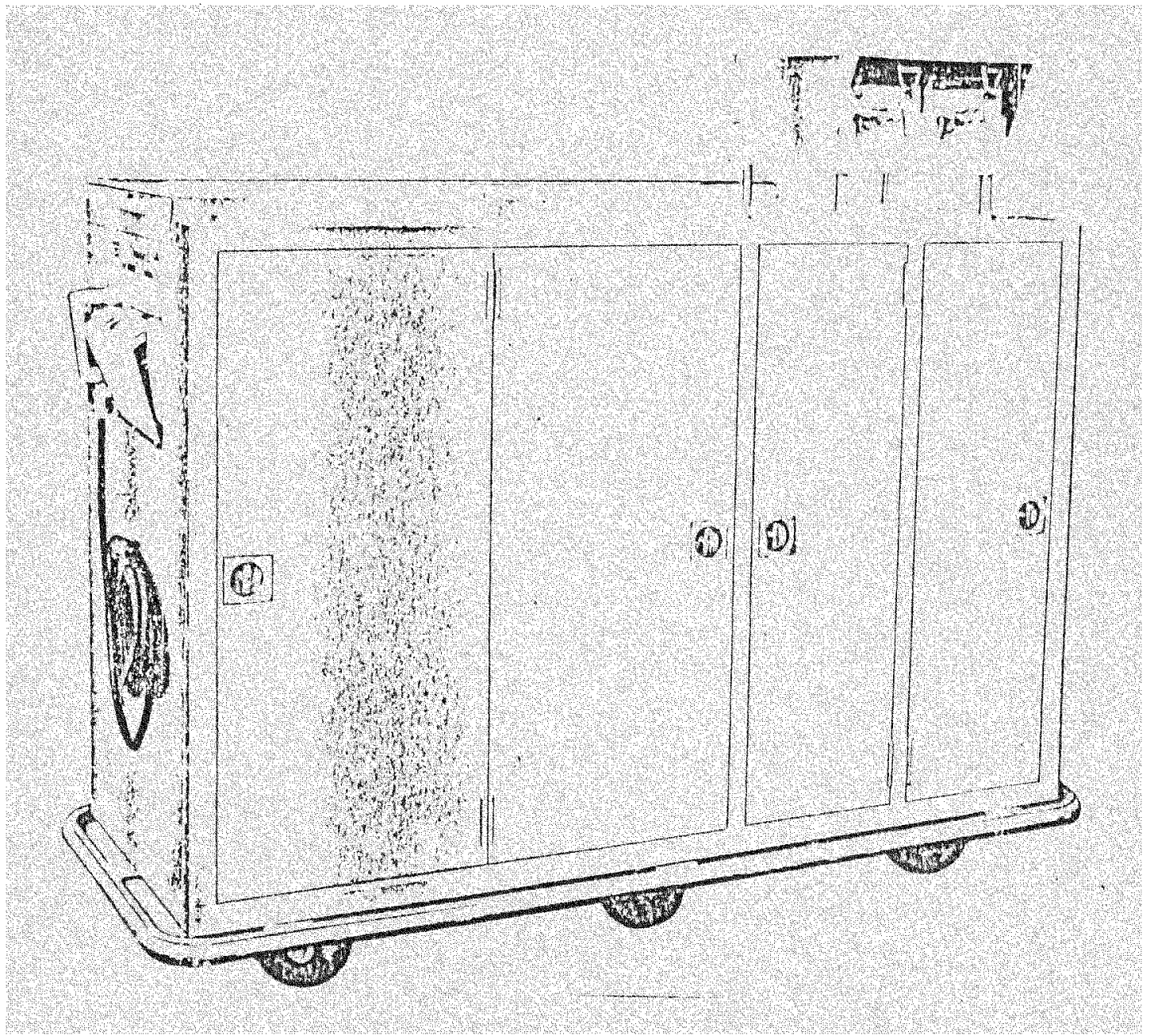

Exhibit $\$ 22$ 


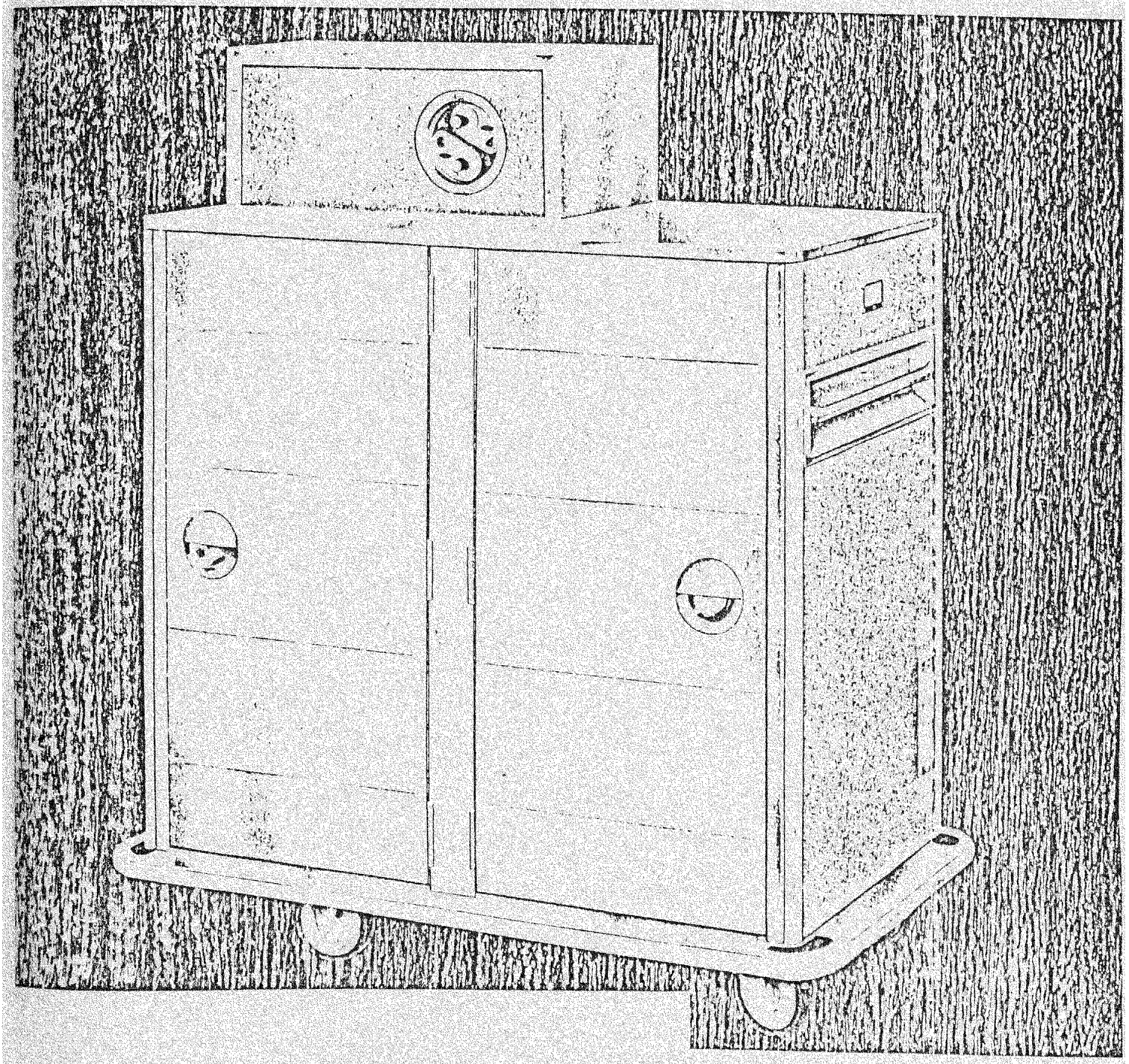

Exhibit 23 
Cost for a 200 bed hospital:

10 Blickman Hot and cold Food

Conveyor

$17 \mathrm{dz}$

Si Lite 15 x 20 Trays $\$ 3,596.00 \$ \$ 35,960.00$

$\$ \quad 47.25$ 803.25 $\$ 36,763.25$

* Does not include china and silverware.

** Toaster and freezer are optional.

Cost per bed is $\$ 183.81$.

Unitray $(\mathrm{tm})$

10

Unitray Heater/Refrigerated

Mobile Cart

$\$ 2,676.00 \quad \$ 26,760.00$

* Comes with trays

** Does not include silverware and china

Cost per bed is $\$ 133.80$.

The manufacturers of the hot/cold carts are:

S. Blickman, Inc.

Crimsco, Inc.

Mercury, Inc.

United Service Equipment co.

other hot cart systems:

The Sweetheart System: (Exhibit 24) The food for this system is assembled in the kitchen on the patients' trays. The hot food is assembled hot; cold foods are assembled cold. The trays are placed in a battery-opexated cart that powers specific heating elements under entree dishes or soup bowls. 


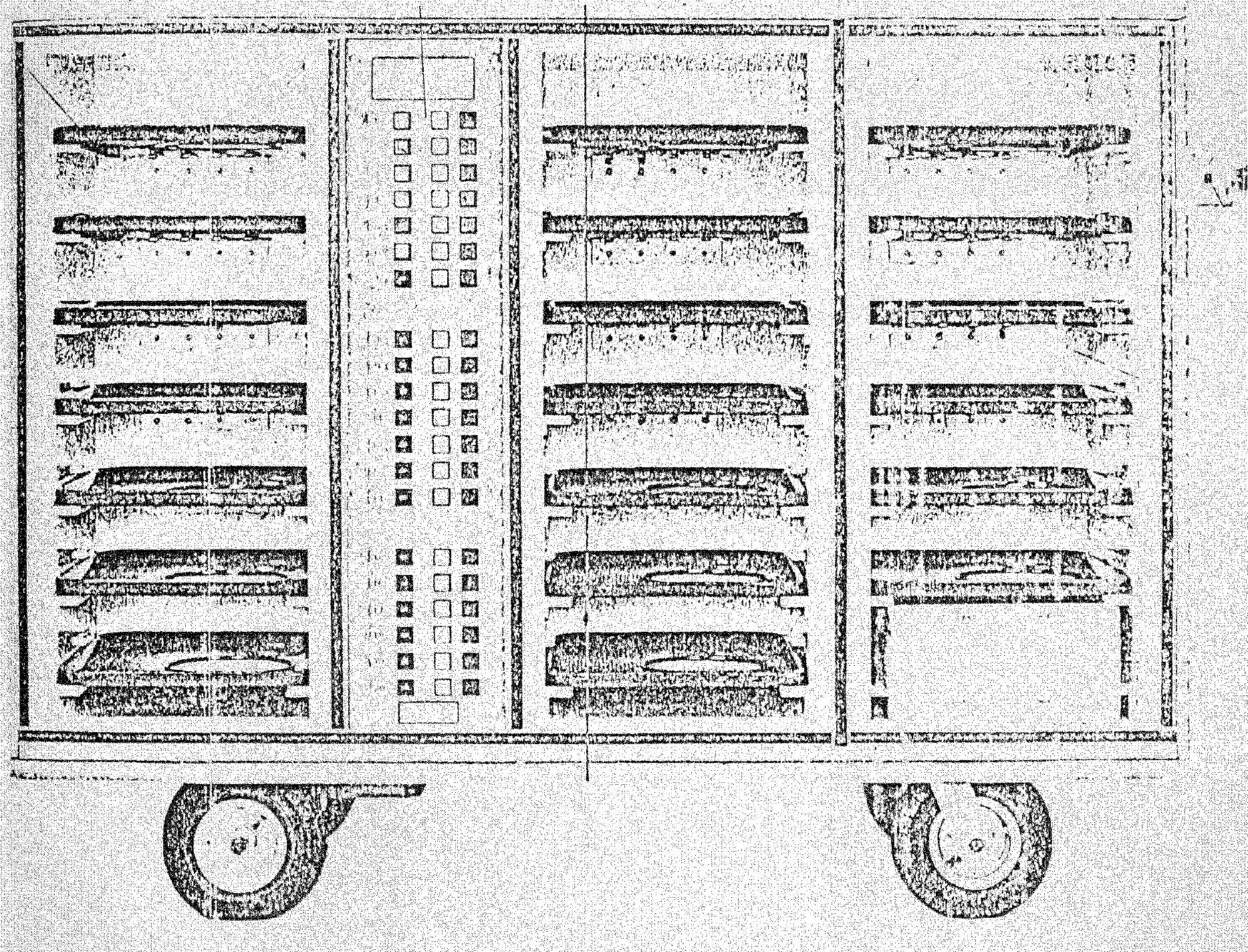

Exhibit \#24 
Copley Square disposable dinnerware from sweetheart is the only dinnerware which will be accommodated by the tray compartments. There is no refrigeration connection with this system. Cold foods must retain their own cold temperature during delivery.

The cart is delivered to the patients' floor in the automatic drive cart. Food can be held approximately $11 / 2$ hours without substantial loss of food quality. Food temperature will remain at 150 degrees $F$. for up to two hours if necessary.

Cart batteries are charged between meals for use at the next meal. The system is sold and/or leased by sweetheart only. The cart costs approximately $\$ 300.00$ per bed. The capacity is 20 meals per cart.

Convenience and "Ready Food" systems have produced a demand for tray delivery carts which are refrigerated. Several manufacturers have made just a refrigerated cart into which the cold plated meal is transported to the patients' floor. The carts are plugged in on the floor. The food is held in the chilled state until the patient is ready for his tray. When the patient is ready, the tray is taken from the cart and the food is end-heated in a microwave, convection oven, re-con or some other type of heating device.

The carts are heavy and hard to handle. They are good for hospitals that have a limited amount of space for making a galley. Some hospitals use the cold carts as a 
mobile microwaving unit. They put the microwave on tope of the cart so it can be transported to the different outlets where the cart and microwave, are plugged in. This is an attempt to serve the food as hot as possible to the patient.

A refrigerated cold cart sells for approximately $\$ 2,600.00$ each. They hold 20 trays.

Another type of cold cart is th- "Drice Cart" and Dietary Products Cold Cart. (Exhibits 25 and 26) Both of these are chilled by dry ice rather than a cooling agent. The dry ice changes the temperature of the freon which is in a closed system within the cart. The freon moves through tubing turning a fan which blows over coils that contain the freon. In effect, it is a self perpetuating machine. The movement of air within the cart gives a more even chilling effect. Both of these carts sell for approximately $\$ 2,400.00$ each.

The Decentralized Food Systems

In the decentralized system in the main kitchen the food is placed in bulk in heated carts, usually one for each nursing unit. One such cart is the Blickman bulk foodveyor which sells for $\$ 2,706.00$. (Exhibit 27) The cart is then sent to a floor pantry or kitchen where the food is portioned out on the plates, the tray is assembled, and then 


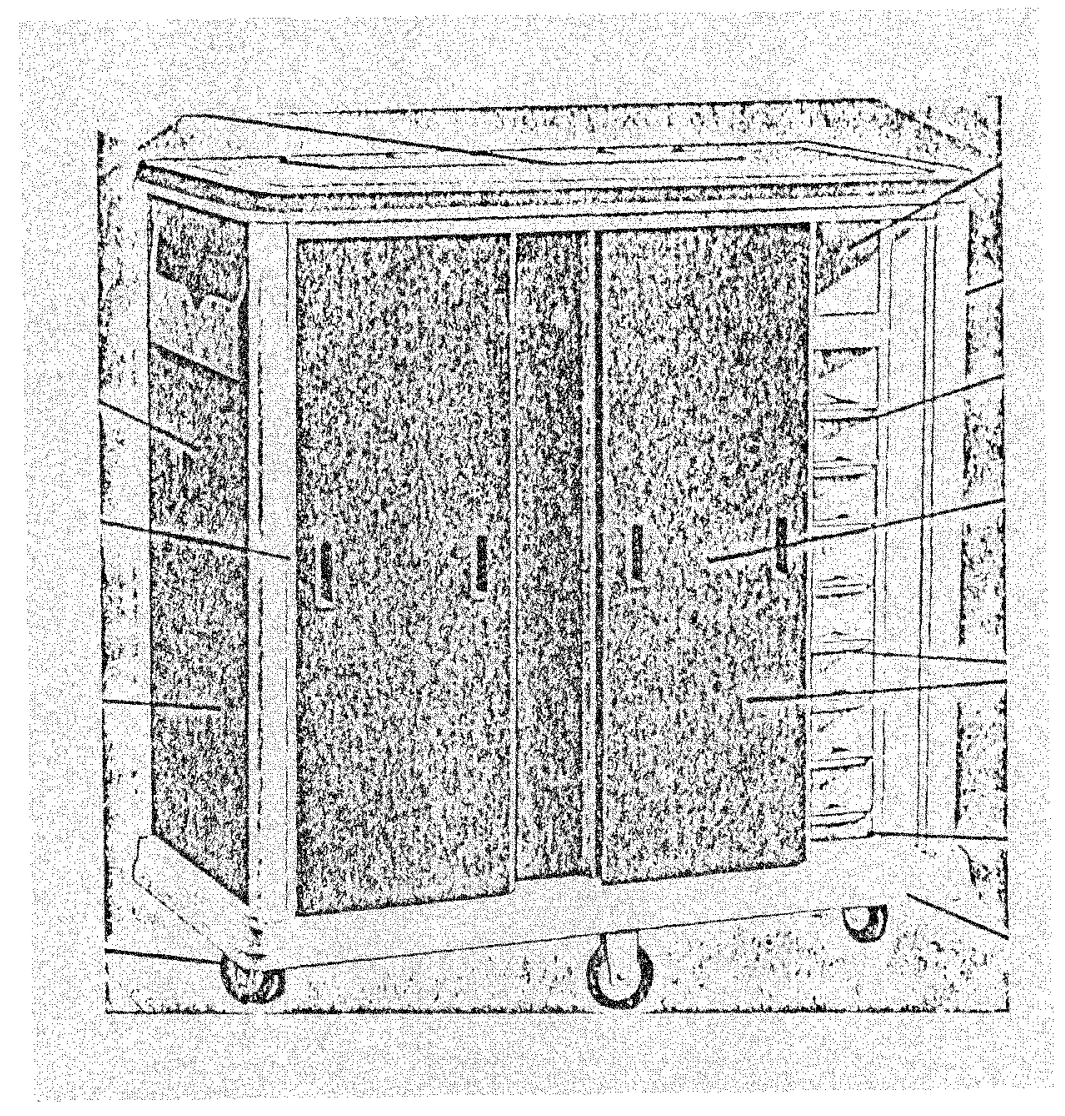

Exhibit $\sharp 25$ 


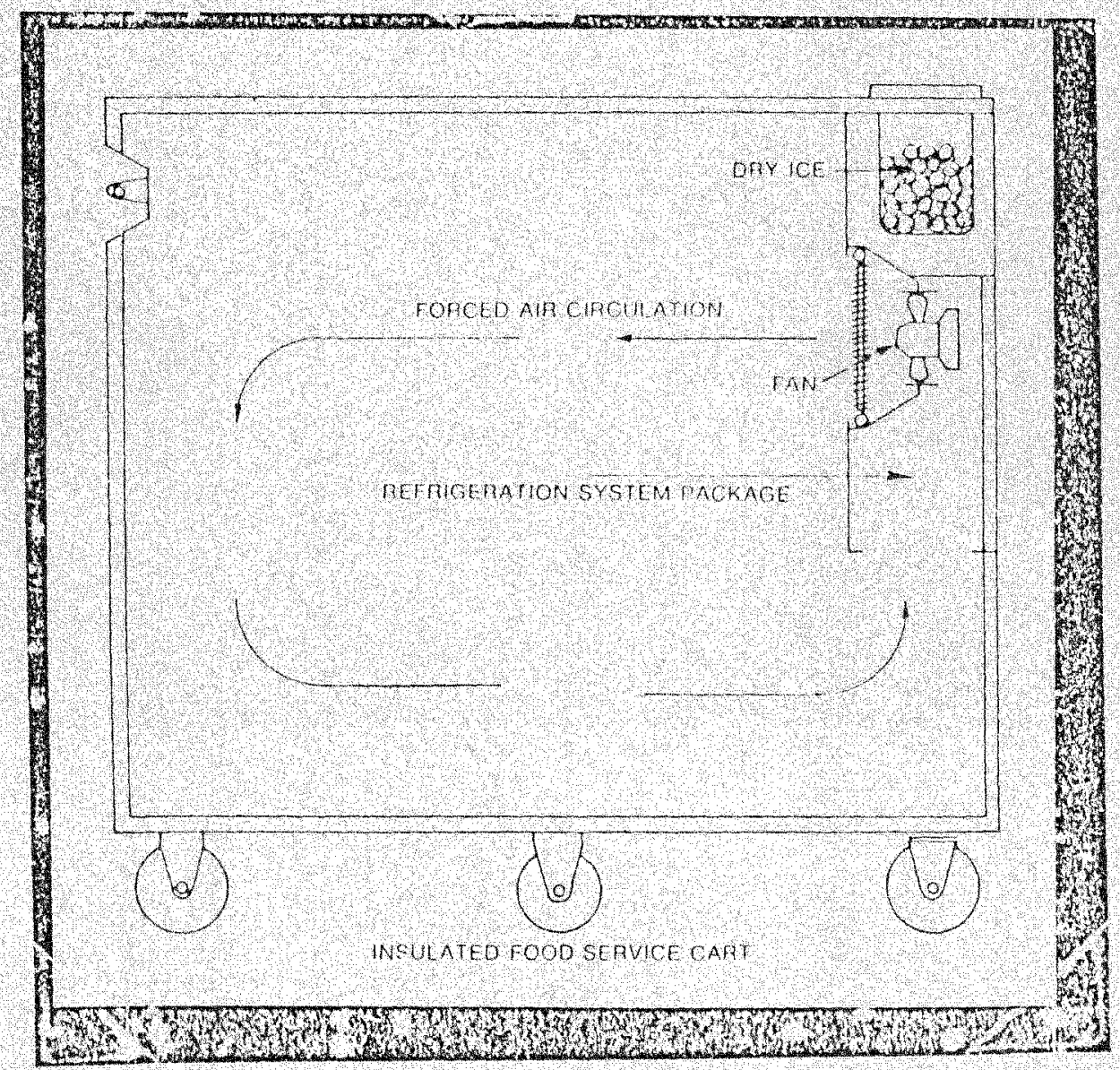

Exhibit $\# 26$ 


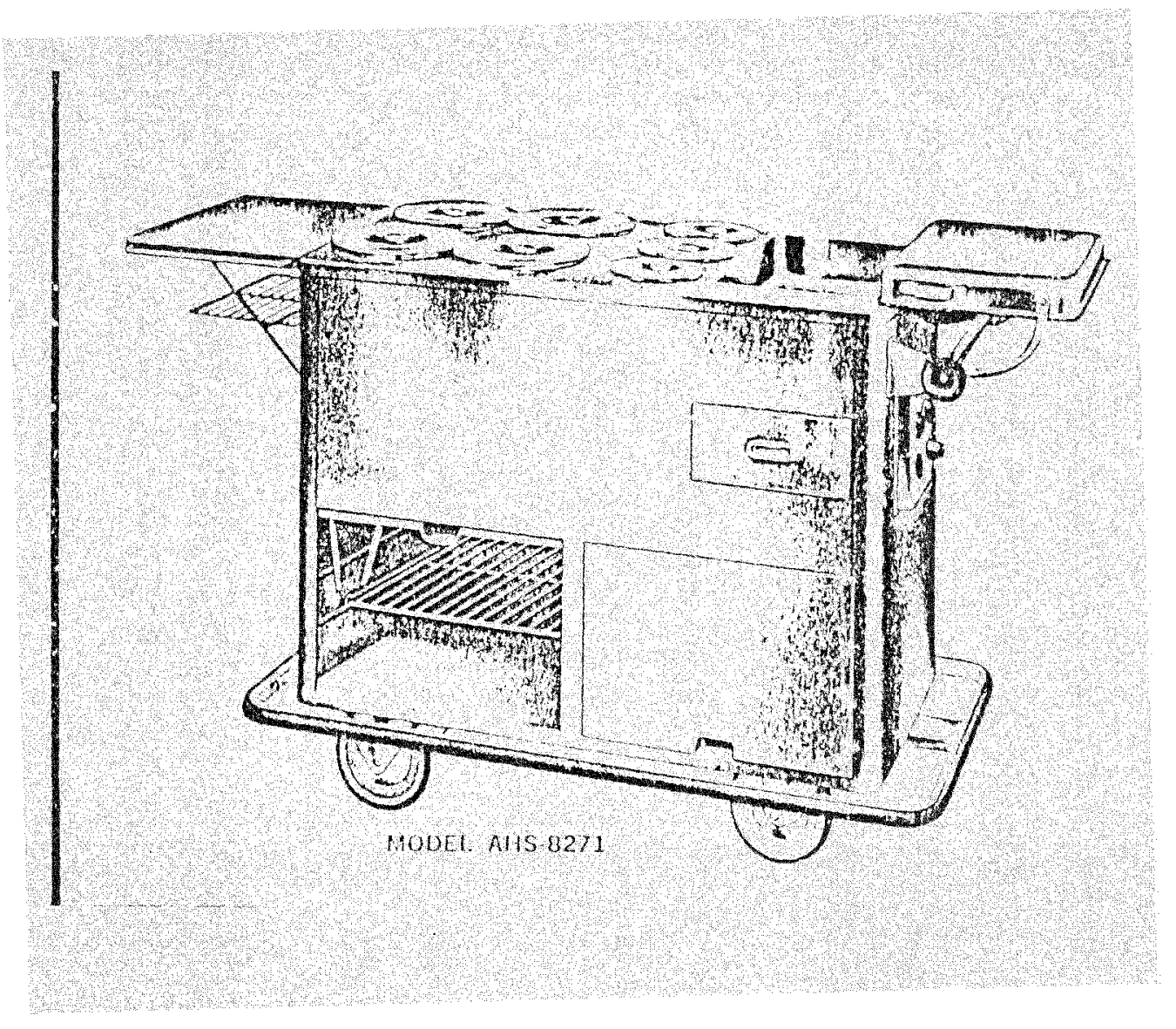

Exhibit \#27 
delivered to the patient. The lack of control, increased labor costs, and improved equipment has caused a decline in this service. 28

Foodservice directors and dietitians have been trying to improve service and heat maintenance yet control portions, diets and temperatures. The galley system is an attempt to meet these goals. Trays are made up completely in a centralized kitchen then transported in the chilled state to decentralized pantries or galleys. The food is end heated in the galley. The most popular method of heating is the microwave oven.

\section{Microwave Ovens}

Microwave ovens are short electromagnetic waves of the same family as those used in radio, television and radar. Although microwaves have no identifiable temperature of their own, they can create temperature rises in food and water. In the same manner as an electrical current throughout the heating element on an electric range, microwaves pass freely through a resistive load. Water is resistant to microwaves and will absorb them, increasing its molecular activity to such an extent that heat is created through internal friction. Thus, heat generated by a microwave oven is the result of microwave interaction with

\footnotetext{
${ }^{28}$ Dietetic Products Division, American Hospital Supply Corp., Catalog, McGaw Park, IL, 1975.
} 
water molecules contained in the food. When the food has been heated for the required time, the oven is shut off, and all that remains of the microwaves is the heated food. 29 (Exhibit 28)

Microwaves, which are in character short, straight waves, are reflected by metals. When transmitted into the oven cavity they are reflected off the metal walls, roof and the floor of the cavity and absorbed by the food. Glass, paper and most plastics are transparent to microwaves and will permit microwaves to pass through them with little or no absorption.

Microwaves cook at a constant power rate, therefore, it is the time setting which must be varied according to the amount of food mass being heated. The microwave oven needs no pre-heating period as does a conventional oven; it requires only ten seconds to warm up before it is ready for operation.

Each patient's meal components should be heated at the same time in separate ovens. For example: soup should be heated in one oven and a sandwich heated in another oven.

${ }^{29}$ Litton Microwave Cooking Products, Hospital Patient Feeding Systems, Minneapolis, MN, 1975. 


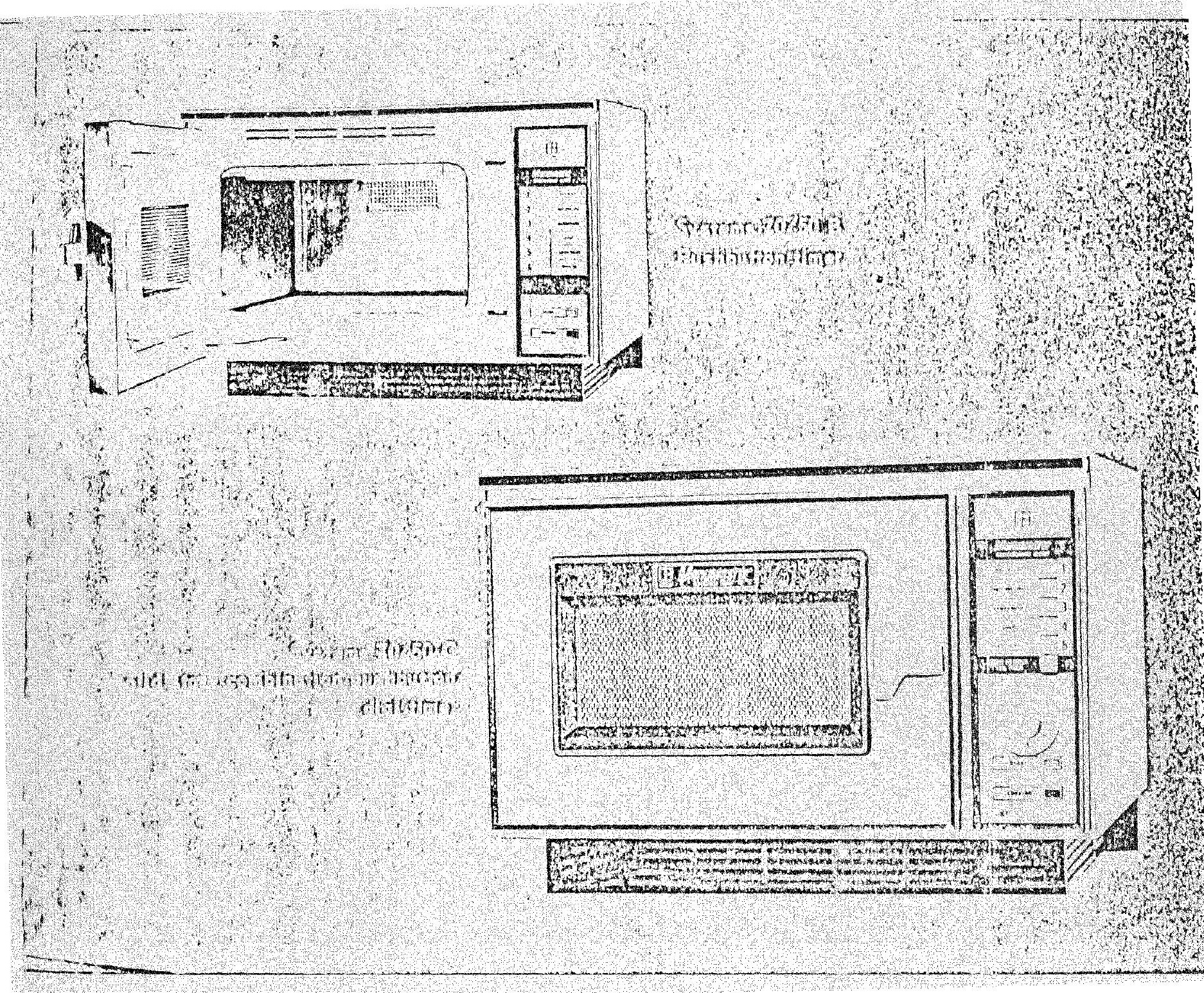

Exhibit \#28 
In a totally integrated galley the following pieces of equipment should be incorporated:

2 Microwave ovens

Holding refrigerator for carts with

made-up trays or a cold cart

coffeemakex (with hot water for tea or chocolate)

Toaster

Icemaker

Freezer

Egg Cooker

Small refrigerator for holding extra supplies

A galley with the proper equipment can serve from 30-40 patients in approximately $35-45$ minutes. As the number of patients increases so will the total time needed to feed them increase if the number of ovens remains constant.

Litton Industries lists the following benefits to a hospital using a microwave system: 30

Greater patient satisfaction

Improved dietary employee morale

Foods tase and look better

Reduction of harmful bacteria growth

Greater flexibility in feeding time

Control using central assembly

Less investment in kitchen equipment

Eliminates peak kitchen volume

Easy to implement when planned

Dietary heating maintains control

Labor is greatly reduced

Microwave is economical to operate

Some of the problems of a microwave system are:

Food must be covered with gravies or a sauce

Safety: pacemakers must be kept away

Plating

More skilled personnel in the galleys

30 Litton Microwave Cooking Products, Hospital patient Feeding Systems, Minneapolis, MN, 1975. 
Problems of a microwave system, cont'd.:

overcooking: eggs are difficult to prepare in a microwave

China service: because patient is fed when he desires

Hard to schedule dishwashing

Manufacturers of microwave ovens are: 31

Amana Refrigeration, Inc.

Food temp, Inc.

Hobart Corporation

Litton Microwave Cooking Products

Magic Chef, Inc.

Toastmaster Commercial Division

Cost for a 200 bed hospital with five (5) galleys:

10 Litton Microwave Model 32 $70 / 40 \mathrm{~B}$

$\$ 1,195.00 \$ 11,950.00$

5

Traulsen Refrigerator 33

RRI . 2-32 L UT

$3,518.00 \quad 17,590.00$

Precision OURT-1422-534

Carts

$186.00 \quad 1,860.00$

*Crimsco Model 843035

Galley Station

$7,299.00 \quad 36,495.00$

Includes:

Storage Cabinets

Coffee dispenser

Icemaker/dispenser

Refrigerator/freezer

overcounter workshelf

Workcounter

Duplex outlets

Full-width lighting

(Exhibit 29)

TOTAL $\longdiv { \$ 6 7 , 8 9 5 . 0 0 }$

*China and silver and trays not included.

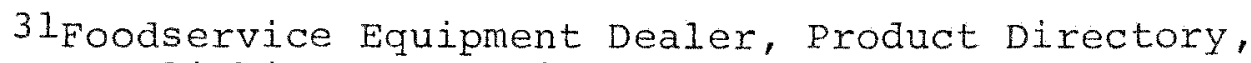
Cahners Publishing Co., Chicago, IL, 1974.

${ }^{32}$ Litton Microwave Cooking Products, Hospital Patient Feeding Systems, Minneapolis, MN, 1975.

33 Traulsen and Co., Inc., Catalog, College Point, NY, 1975.

34 Precision Metal products, Inc., Catalog, Miami, FL, 1975.

${ }^{35}$ Crimsco, Inc., Catalog, Kansas City, Mo, 1975. 


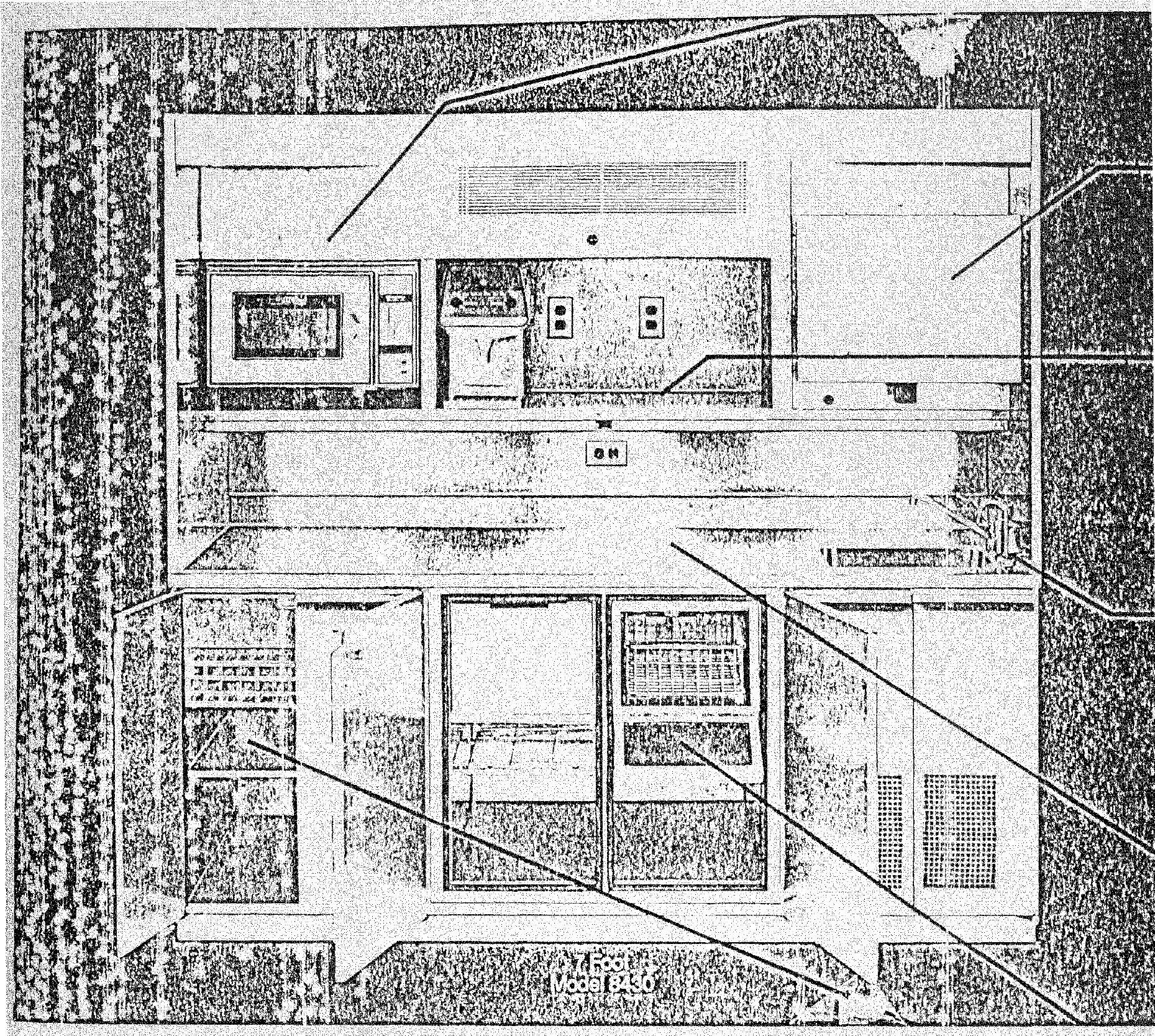

Exhibit \#29 
Cost per bed is $\$ 339.47$.

Cost could be reduced by using a cold cart and eliminating the refrigerator and carts. The cold cart system has more problems as far as maintenance is concerned and they are hard to maneuver around.

There are several other galley type systems on the market using variations of the microwave oven or some other method of rethermalizing the food.

The $3 \mathrm{M}$ Integral Heating System

(Exhibit 30) Patients' trays are assembled in the kitchen with cold food placed on specially designed 7" square plates. The soup and cereal container is $4 " \mathrm{x} 5$ ". The special plate contains electrical heating elements that connect to the four contact points on the base. Patient trays are assembled in a cold state in a centralized kitchen. The hot portion of the meal is removed from the patient trays are assembled in a cold state in a centralized kitchen. The hot portion of the meal is removed from the patient trays and placed in the module oven. The plates are overwrapped or covered with a lid. The module is turned on and the meals are heated by electrical current slowly pulsating to heat the plate to 180 degrees $F$. The food on the plate and in the bowls is then reheated by conductive heating - heat transferring from the plate or bowl to the food. 


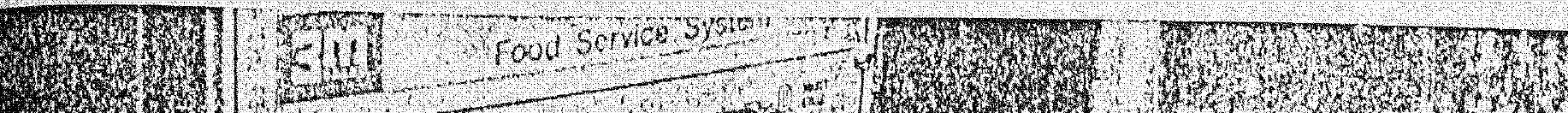
8.1. 474. (6),

. (2) intis 2. (1) (2)

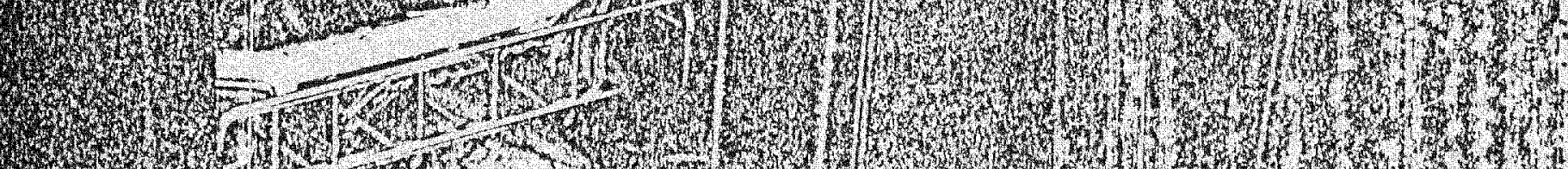
2014 2. 7) -

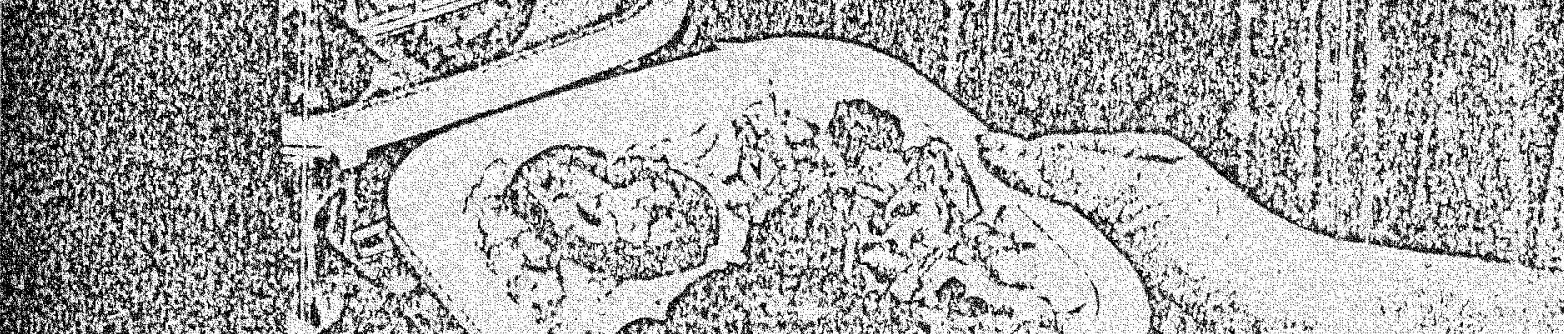
(3)

(x)

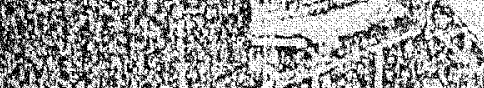

$202.2+3$

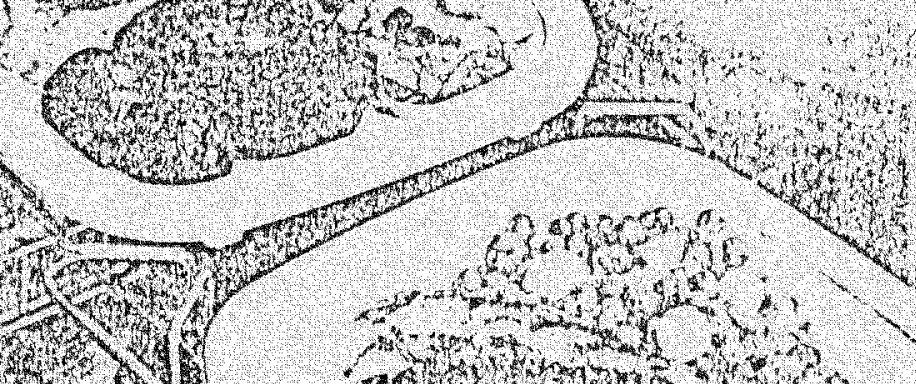

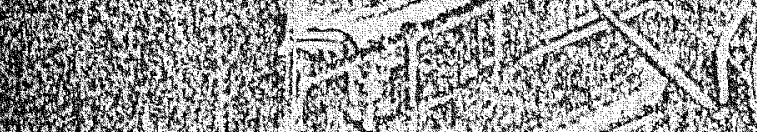

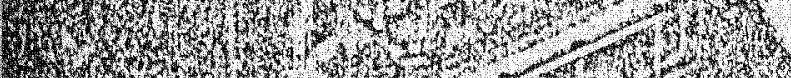

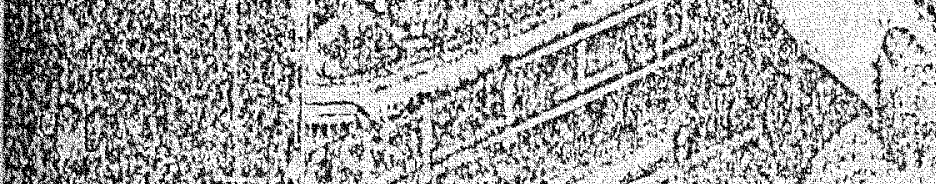
H.

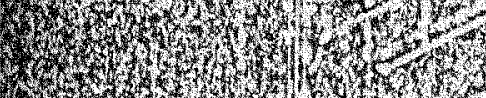

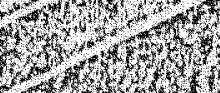

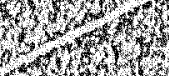

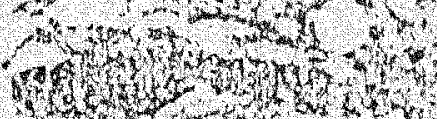

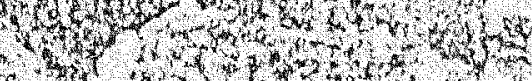
(x) 3.t.

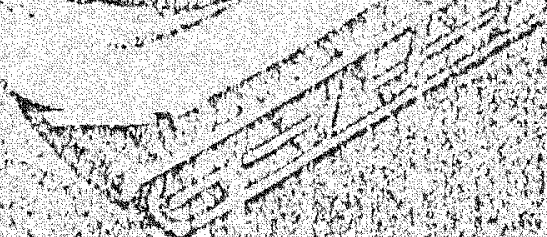


The heated portion of the meal is then returned to the patient's tray by matching the meal with the tray by either number or name. The heated meals not served due to patient delays can be held hot in the module or the plate will hold the temperature on the tray for 15-20 minutes because of the insulating properties of the container. This system is leased only for a cost per meal of 96 to $11 \%$ plus an $\$ 80.00$ per month charge for the use of the module. Any special plates that do not operate properly are replaced by the manufacturer. Price includes service of the modules.

Each module accommodates 24 meals. Typically, two modules per nursing station or pantry unit are required. The heating time from a 40 degree $F$. holding state is 12 minutes. The only plates that can be used with the system are supplied by $3 M$ along with the modules.

\section{Advantages}

24 plates can be heated at one time in 12-15 minutes

Uniform heat distribution

A mixture of items can be rethermalized at one time

The $3 \mathrm{M}$ plate retains

heat for a short

period of time
Disadvantages

Extremely expensive

Portion sizes must be exact

Dishes break or leak easily

Complex training is required

All meals must be rethermalized together

Reassembly errors occur easily

The Food Chariot system

(Exhibit 31) This system utilizes the concept of chillplates in a centralized kitchen. Meals are kept in a semisealed atmosphere, $i . e .$, in a special porcelain plate under 
STAINLESS STEEL COVER OR BLACK COATED ALUMINUM)
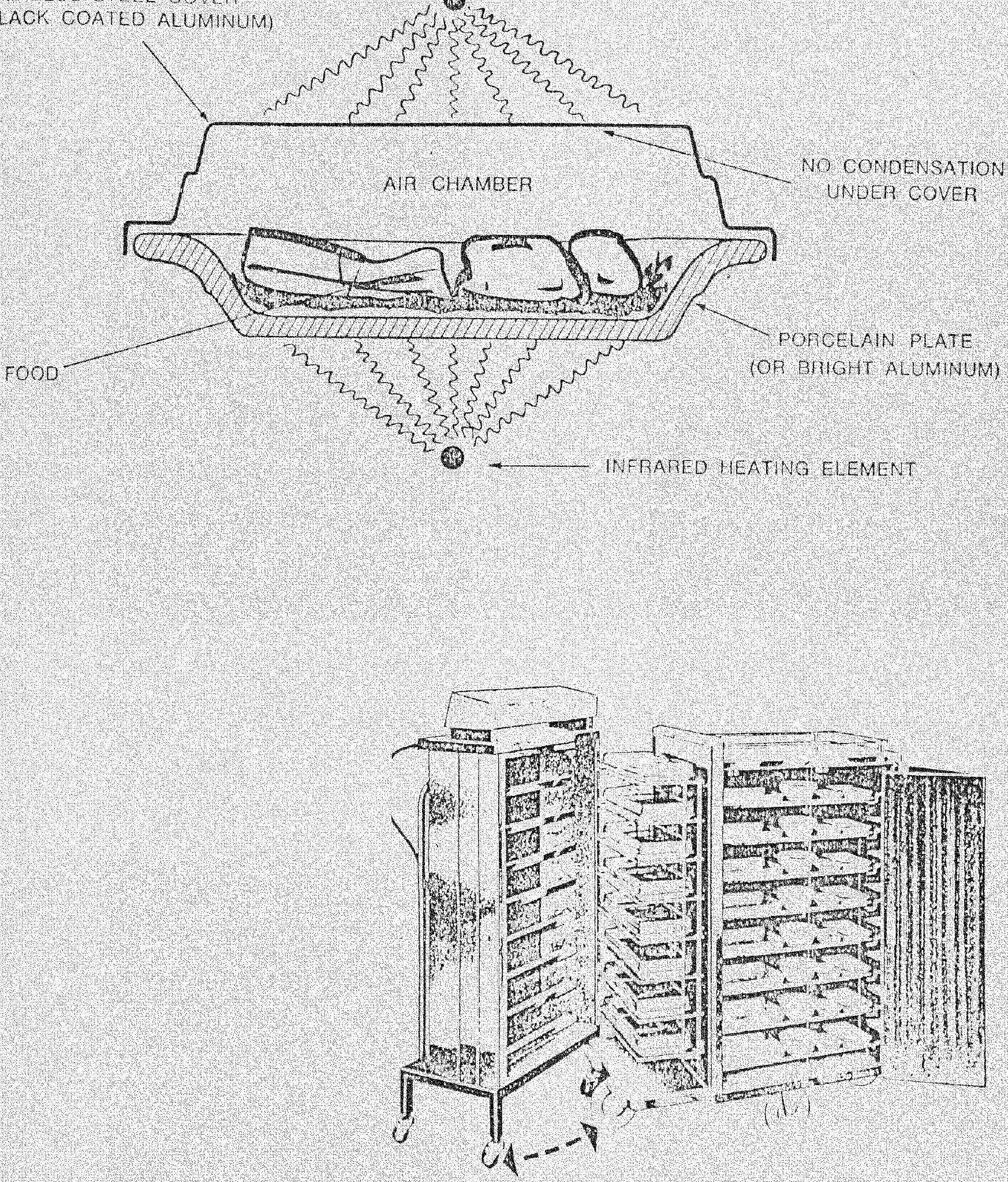

Intrared Cabinet 
a stainless steel dome. Hot portions of the meal are placed on the exterior of the cart on an "oven" rack assembly. Cold items are placed on trays in non-refrigerated carts (capacity 16 or 20 trays). Mobile carts are stored in refrigeratore until mealtime.

At mealtime, carts are delivered to pantry units where the heatable section of the mobile cart is backed into an infrared heating device.

Three groups of infrared tubes radiate dark infrared heat producing soft, progressive and uniform heat in the entree plate/dome assembly, thus rethermalizing the meals. The average time for rethermalizing is 12 minutes for refrigerated foods (40 degrees $F$.$) and 24$ minutes for frozen foods.

This system is expensive $(\$ 300.00$ per bed) and equipment intensive (plates, domes, carts and heaters are all necessary; also need pantry or refrigeration storage units).

\begin{tabular}{cc}
\multicolumn{1}{c}{ Advantages } & Disadvantages \\
Rethermalization of & Mobile carts are not \\
l6-20 meals at one & refrigerated \\
time & Expensive - $\$ 300.00$ per bed \\
Infrared heating & Limited control of individual \\
equipment saves the & temperature \\
integrity of the & Reassembly can cause mistakes \\
food-limited & Entrees are only semi-sealed \\
"cooking damage" & during transport to the \\
& pantries \\
& A heat-oriented system - \\
& insufficient cold retention
\end{tabular}


(Exhibit 32) The concept behind the Alpha System is heating chilled food in a cooled atmosphere in 32 minutes. A completely set tray is placed into a cart on tray slides that have three heating plates on the left side. The tray has three cut-outs to hold the hot food dishes so that they can touch the sides / heating plates. As the tray slides into place, the dishes of hot food are raised about $1 / 4$ " in order to get them off the tray and therefore not conduct heat to the other items on the tray. The cooling unit is similar to the Crescent Metal "cold pak"; refrigeration is wal1-mounted and not affixed to the cart. The air is circulaterinto the cart and back out to the refrigeration unit. Each cart will accommodate 20 trays with 4 " spacing. At a weight of 500 lbs., the cart will be approximately 47 1/2" long by 26" wide by 54" high. It is electrically powered and has a range for cold foods of 34 to 38 degrees F. Totally insulated, these temperatures can be held with a good degree of accuracy.

Either reusable or high heat disposables can be used. Cold food is loaded on a centralized tray assembly Iine. Immediately, the carts are hooked into the refrigeration or air conditioning unit either in the kitchen or on the floor pantries. Food can be held in the refrigerated state over night or longer. 32 minutes prior to serving, the cart is turned on. The hot foods were previously oriented with the hot plates so that 32 minutes later the food is hot. If the 


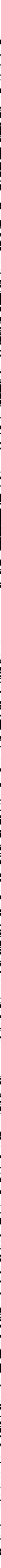

Exhibit \#32 
patients are not ready to eat, the cart automatically switches to "holding" temperatures at the end of the 32 minute cycle. This will hold food in good condition for up to 3 hours. Delivery is made from a pantry or by wheeling the cart to each individual patient door. Soiled dishes are returned in the same cart to the dishwashing area.

\section{Advantages}

Easy to operate once the system leaves the kitchen $\underline{\text { Disadvantages }}$

Little control over temperature of different portions and types of food

Hot beverages must be handled from the floors

Insulated bowls must be used for ice cream; if long term refrigeration is used, ice cream must be added at mealtime

Special plating required for thick and tender items

Food must be thawed $\left(40^{\circ} \mathrm{F}\right.$.) for rethermalization

Potential maintenance problems probably expensive.

The Chemetron Votator Total Tray Microwave (Exhibit 33) Patients' meals are assembled in the kitchen on a specially designed tray. Food that is to be heated is placed on the front part of the tray in a defined area; food to remain chilled is placed in the back of the tray in a defined area with silverware, condiments, etc.

The tray is equipped with a magnetic sensor used to determine the proper heating time of each meal based on how 

much food is on the hot portion of the tray. The trays with all of the patient's meal except hot beverage, are refrigerated between plating and rethermalization.

The entire tray is placed in a specifically modified microwave oven (Litton 70/80). A metal shield automatically drops with closure of the door over the cold portion of the tray. The hot portion of the meals are heated in three cooking stages determined automatically by the sensor in the tray. When the food has reached the proper temperatures as determined by the sensors, the microwave oven shuts off automatically.

Most types of china and microwaveable disposables can be used. The plate dimension for the hot portion of the meal cannot be larger than an 8" round or a 5" $x$ 8" rectangular plate. Geometric plating of items is still necessary as is true of all regular microwave ovens.

This system is leased only on a $9 \xi-11 \xi$ per oven usage that is determined by a counter on the oven door. The oven is leased at a cost of $\$ 80.00$ to $\$ 90.00$ per month including service for a minimum of two years. The trays are supplied with the system and damaged trays are replaced at no cost to the user.

\section{Advantages}

No reassembly on the floor

Automatic sensing of food temperatures

Immediate delivery to the patients-virtually the hottest possible food
Disadvantages

Geometric plating is necessary Leased only

Expensive per meal cost per oven usage ( 9 \& to $11 \xi$ )

Additional expense of refrigerated holding equipment is needed for trays 
(Exhibit 34) Patient trays are assembled in the kitchen with the cold food placed on an 8" round or 5" by 8" rectangular plate. Pre-plated meals supplied by food manufacturers can also be used if the food is on a high temperature plate.

The hot portion of the meal is stored on an oven rack cart which includes a lower tray storage area. The entire rack is placed in a refrigerator in the kitchen until meal time.

Prior to meal time, the racks are delivered to the patient floors. The oven racks are slid into the convection ovens mounted at the same level as the top of the cart and trays are rolled into the refrigerator below.

The pre-plates are covered and the convection oven is turned on to 250 degrees $F$. The meals are heated in about 15 to 20 minutes. The meals that are not to be heated because of patient delays are removed prior to heating and placed in a refrigerator to be heated later, as needed in a microwave.

When the meals are hot, they are placed on the patient trays in the same order that the trays are placed in the rack. The meals are identified by number or name ox diet to be sure that the patient receiver the correct meal.

The system is sold as a complete galley pantry. Modular in design, it can service 12 to 72 patients, de- 
WESLEY HOSPITAL MEA, GALS, sYSTEM

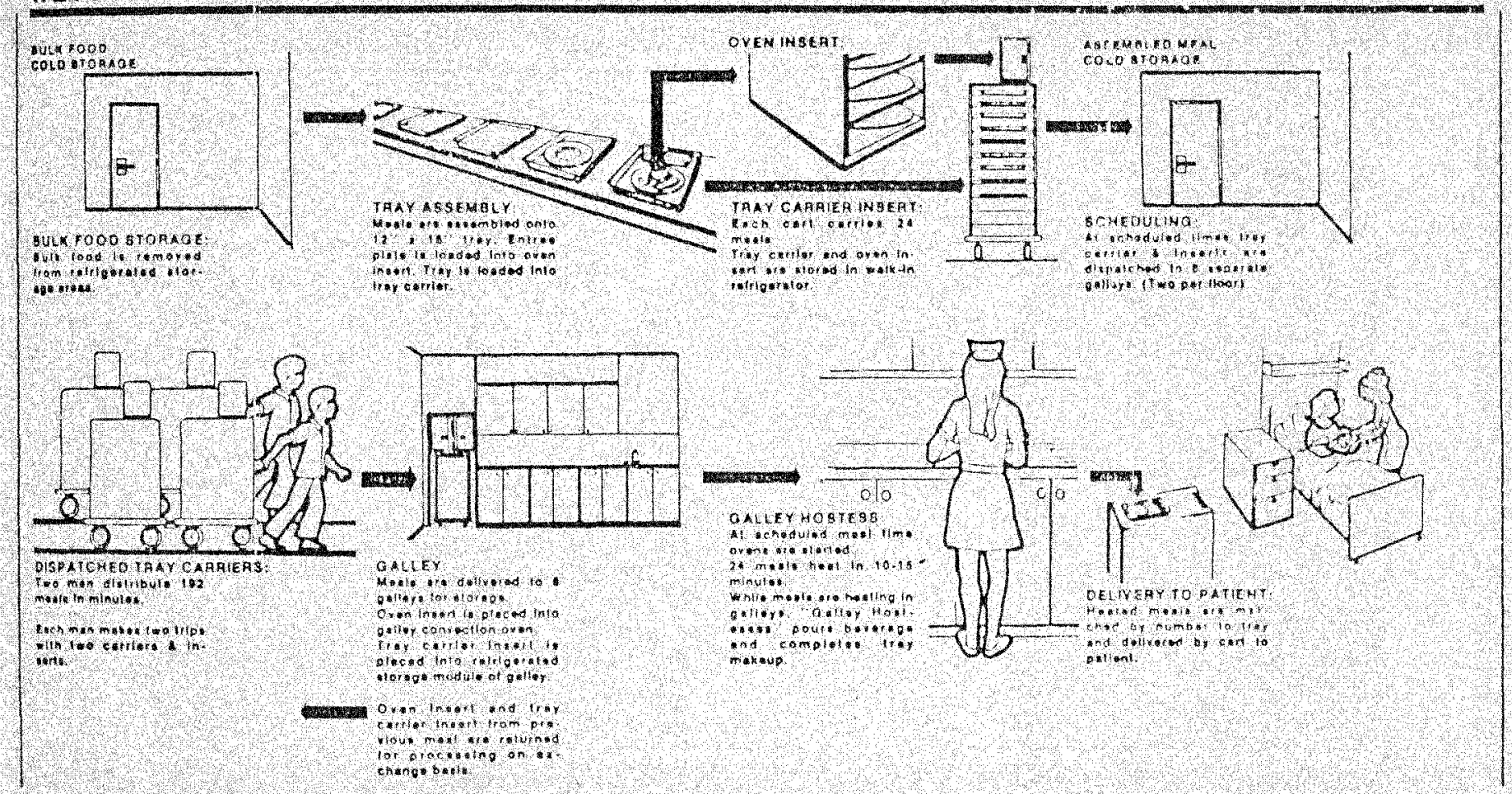


pending upon the number of ovens. Ovens are sold for $\$ 1,000.00$ each for a 24 unit module. The total pantry cost including all components sells for approximately $\$ 6,000.00$. Meals per ovens vary as to the dish size. The oven accommodates 12 round or 18 rectangular dishes. Up to 4 ovens per station can be accommodated to the galley unit. Heating time from a 40 degree $F$. holding state is approximately 18 minutes.

\section{Advantages}

60-72 entrees are heated at one time A minimum of 15 minutes required for total rethermalization High temperature dispos$a b l e s$ or reusables may be used

\section{Disadvantages}

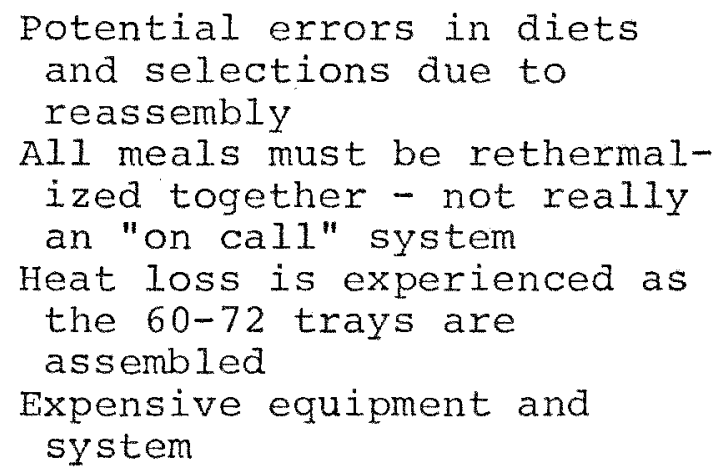

Expensive equipment and system

The Foster Re-Con Oven

(Exhibit 35) The Foster Re-Con oven is used in a galley type operation to rethermalize the foods that should be hot. Based on a patented system of controlled pulsed radiant energy and refrigeration, it is a reconstitutor of prepared and fresh frozen foods, both packaged and unpackaged. It is a holding freezer, a warming unit, plus a broiler. It is also a high air speed oven.

The unit can store from 100 to 1500 frozen portions at 0 degrees $F$. until needed, then, simply by turning a dial, 


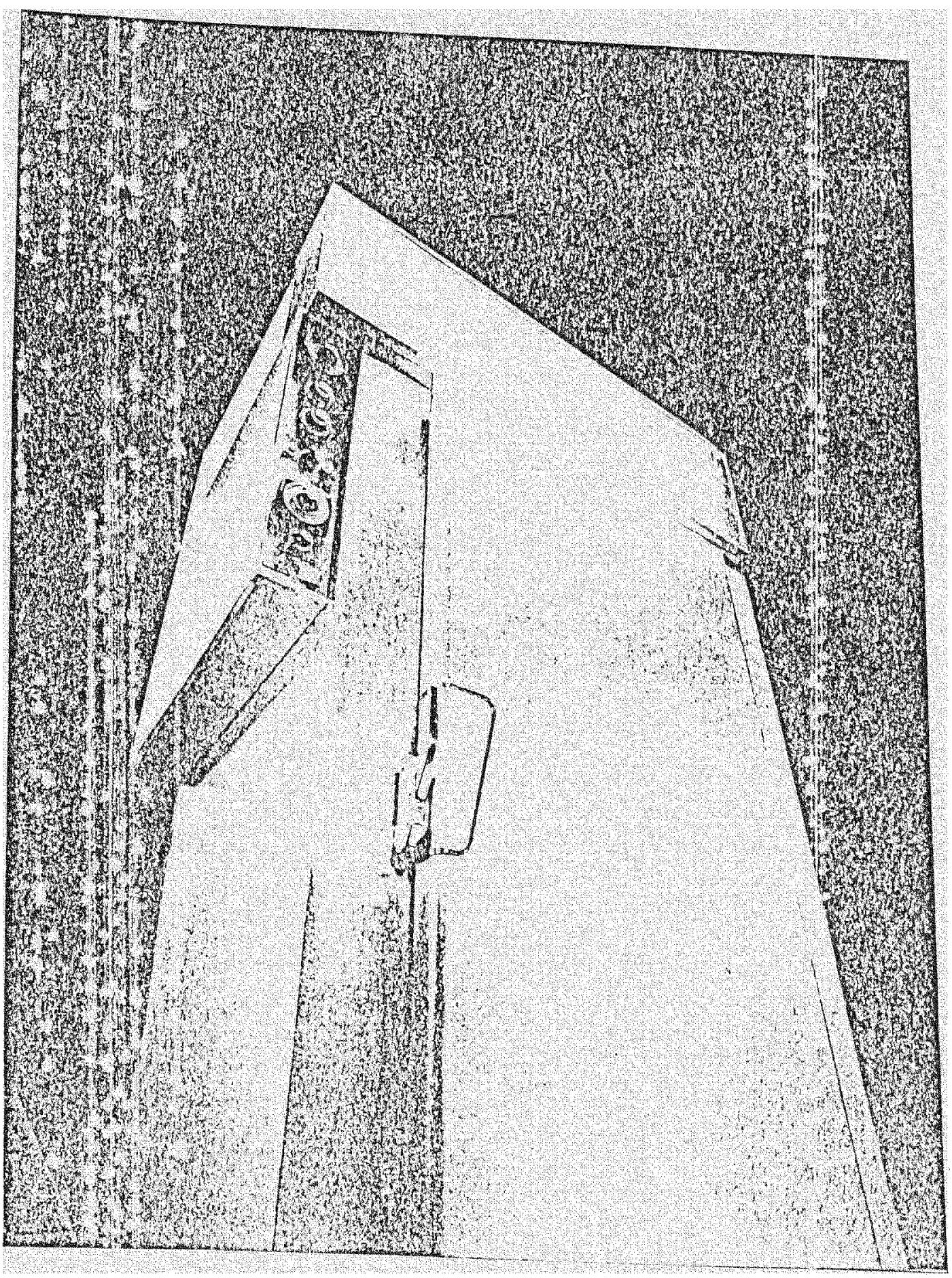

Exhibit \#35 
it reconstitutes them to serving temperatures in minutes. Or, it can immediately reconstitute from external storage freezer (no previous thawing necessary).

The Re-con has been used to reconstitute preplated frozen meals. The cost of the oven ranges from $\$ 4,000.00$ to $\$ 10,000.00 .36$

\section{Advantages}

Fast reconstitution of food

Many meals can be prepared at one time Flexibility, Convection or Re-Con oven

\section{Disadvantages}

Very complicated to operate Flavors bleed to other foods being prepared at the same time

Expensive

Must have a container which can withstand the high temperatures and changing temperatures

${ }^{36}$ Foster Refrigeration Corporation, Catalog, Hudson, NY, 1975. 
CHAPTER III

TRAY ASSEMBLY SYSTEMS

The tray assembly system is like the assembly line in an automobile manufacturing plant. It is the place where all of the parts come together to make the finished product. In a hospital which has to feed large numbers of people special and varying diets in a short period of time, the tray assembly system is the backbone of the operation.

In the past, food was taken in bulk to the patient's floor to be dished out and distributed. This proved to be a troublesome system because of lack of control, mistakes and rising costs. The centralizing of tray assembly solved these problems plus improved the time and quality of service. Both hot and cold plating is being done in the central kitchen. This is accomplished by moving the tray down a production line with different stations. At each station someone places an item on the tray. The usual set up has the following stations:
Starter
Entree
Salads
Desserts
Beverage
Checker
Loader

Staffing varies as to the size of the hospital, type of menu, delivery system, meal and efficiency of help. 
There are five types of conveyors used in hospital tray assembly systems. They are:

The Manual slide Line
The Skate Wheel Line
The Roller Conveyor
The Belted Line
The Tray Type Iine

The use of each varies according to the size of the hospital and the type of menu being used.

To determine which is best suited for the particular account, a survey must be taken of the size of the institution, the type of heat maintenance system used, the space available and flow patterns of the institution. The number of trays which are to be served must be calculated. This is done by taking the number of meals and dividing it by the time period used to serve the tray. The distance that the tray must travel or the time it takes from the central kitchen must be taken into consideration. This factor is not as critical in a cold plating operation.

\section{The Manual slide Line}

The manual slide line is used in smaller hospitals, usually under 250 beds. It gets its name from the way the trays are moved down the line, manually. The line can be in the kitchen or even in the cafeteria. The trays are pushed on tray slides from person to person. One such line is the Shelley system. It consists of: 
1 ea TT2 1520 Tray Starter Unit

2 ea pCSC 48 Refrigerated serving

Counter

1 ea PCS 48 Coffee Counter

2 ea PHC 3 Hot Food Counter 36

$\$ 654.00 \$ 654.00$

$1,638.00$

$3,276.00$

943.00

943.00

$1,814.00$

$3,628.00$

(Exhibit 36)

The counters are held together by an interlock machine mechanism. The electrical requirements are two 280 volt lines for the hot food counters and two 115 volt lines for the refrigerated counters.

250 trays can be produced in 60 minutes using six people. The staffing of the line is:

I starter

1 cold food person

1 hot beverage person

1 hot food disher

I soup and hot cereal disher

I checker and loader

This type of set up is good in small hospitals but it has its drawbacks. There is no motivating factor of a moving line to speed up the operation. Personnel must use their hands to push the trays so they cannot use them for preparation or gathering for the next tray.

\section{The Skate wheel Line}

The skate wheel Iine was designed to save time, labor and money in any food service operation. The skate wheel line is designed to carry flat bottomed trays. The skate wheel line comes in lengths from 8 feet to 20 feet. They are available in top mounted or mounted on stanchions.

${ }^{36}$ Shelley Manufacturing Co., A Division of Alco Food Service Equipment Co., Miami, FL 1975. 


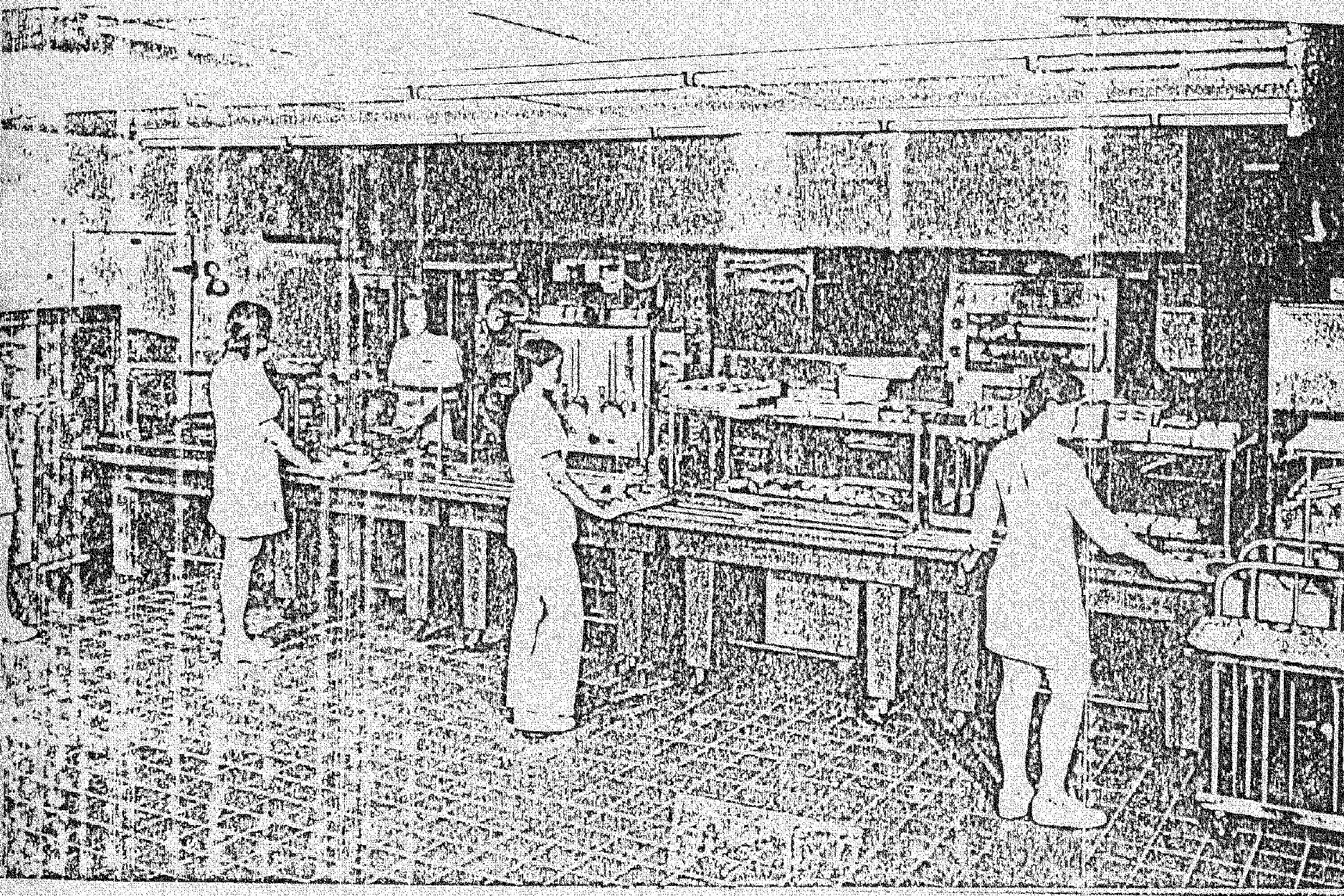

Exhibit \& 36 
Electrical chase with outlets and circuit breakers can be installed to fit the needs of the hospital. (Exhibit 37)

This line can be used with either hot or cold plating operations. The support equipment would be rolled to the conveyor where the personnel would fill the respective orders. The trays are pushed by hand. The wheels speed up the operation over that of a manual line.

One such line is the Precision HI-Speed "Compact" tray assembly system. It consists of:

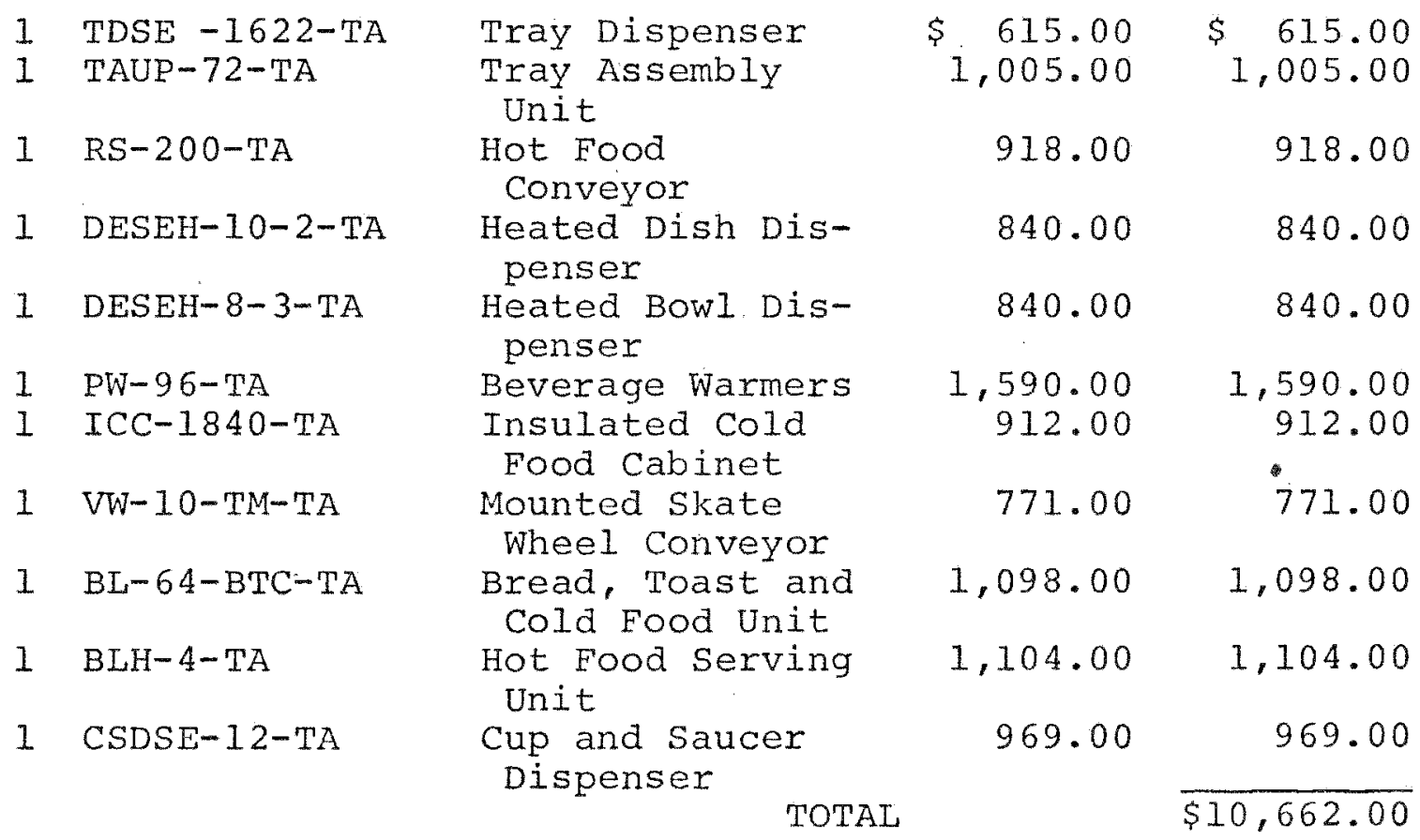

Precision Metal Products, Inc., claims that three people can make up 300 trays per hour using this system, 38 but in most hospitals more than 3 people are needed. (Exhibit 38)

38precision Metal Products, Inc., Catalog, Miami, FL, 1975. 


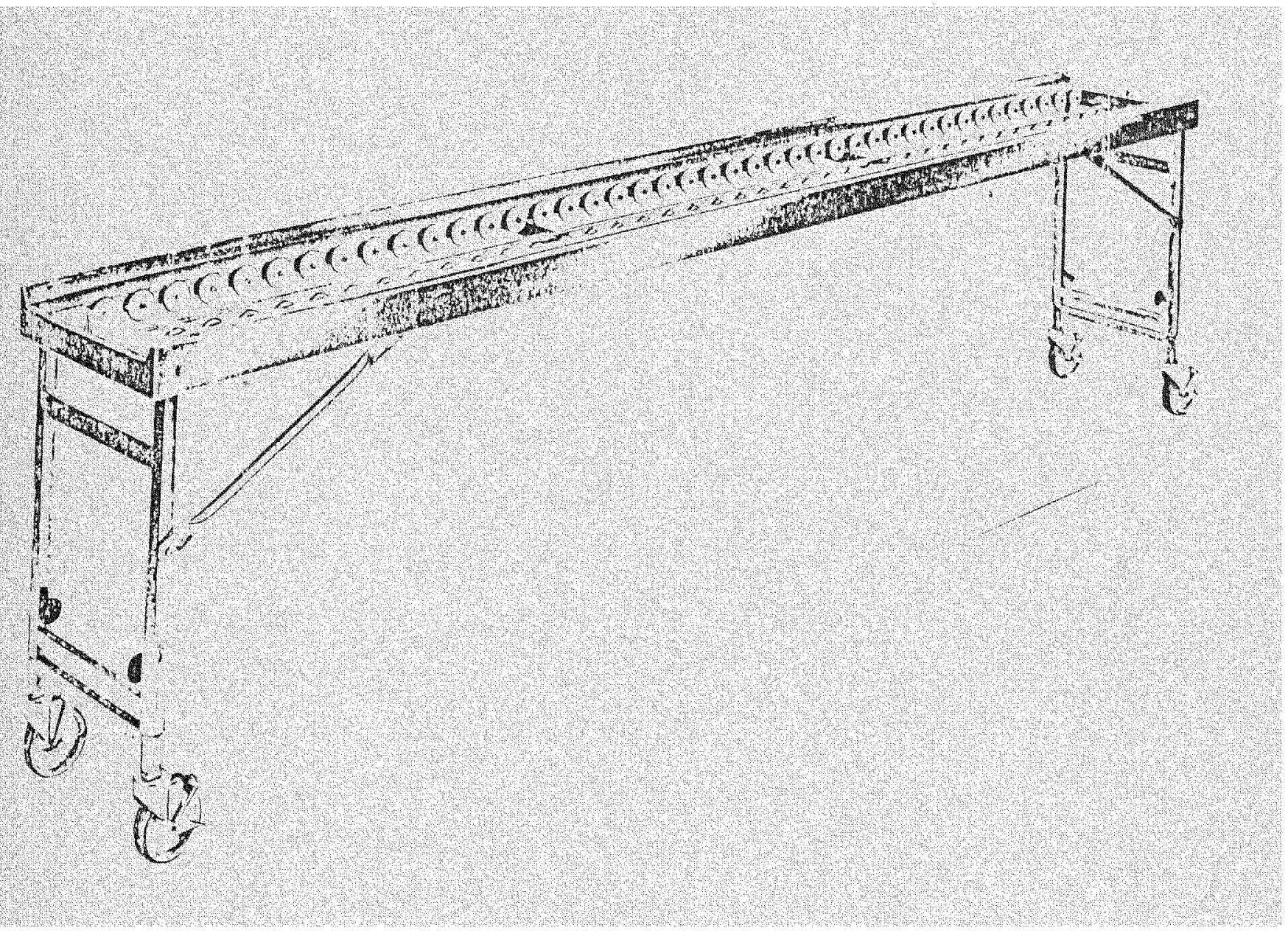

Exhibit \#38 


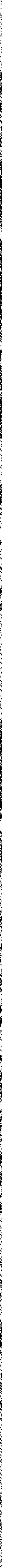

19, H.t.

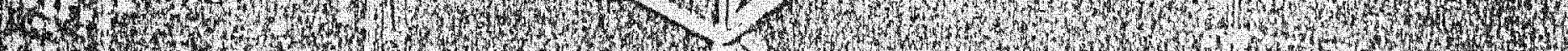
7, 196, 
The Roller Conveyor

The roller conveyor Iines operate in the same way as the Skate Wheel assembly line but it can be used for compartmented as well as flat bottom trays. (Exhibit 39)

\section{The Belted Line}

A motor driven belt is the mechanism that carries the trays in this system. The belt is either continuous nitrile-polyester or flexible polycarbonate compound. The Iine has either fixed or variable speeds. It can be stationary or mobile. Electrical raceways can be plugged into the line, eliminating many outlets from the floor. Automatic belt washers are available for easy cleaning. The belted line is equipped with a micro switch at the end in order to stop the Iine when a tray gets to the end. The belted line can be used for hot or cold assembly. (Exhibit 40)

The belted line frees the employees from manually pushing the trays down the line. This gives them more time to concentrate on the items that will be put on the tray. The belt is a motivating factor because the employees feel that they must keep up with the speed of the belt.

A belted tray assembly line for a 200 bed hospital using a centralized hot food system would have the following equipment: 


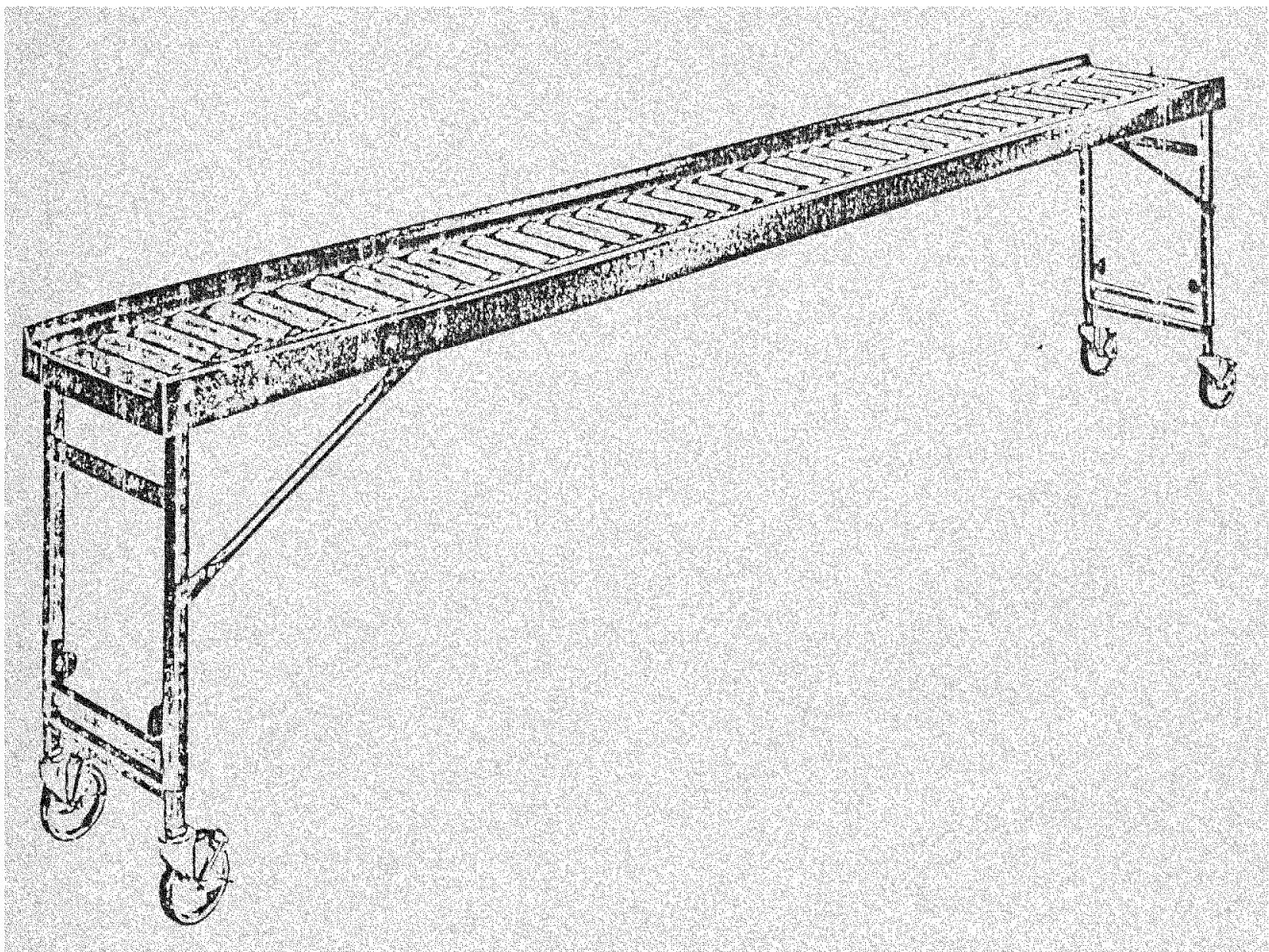

Exhibit 非39 


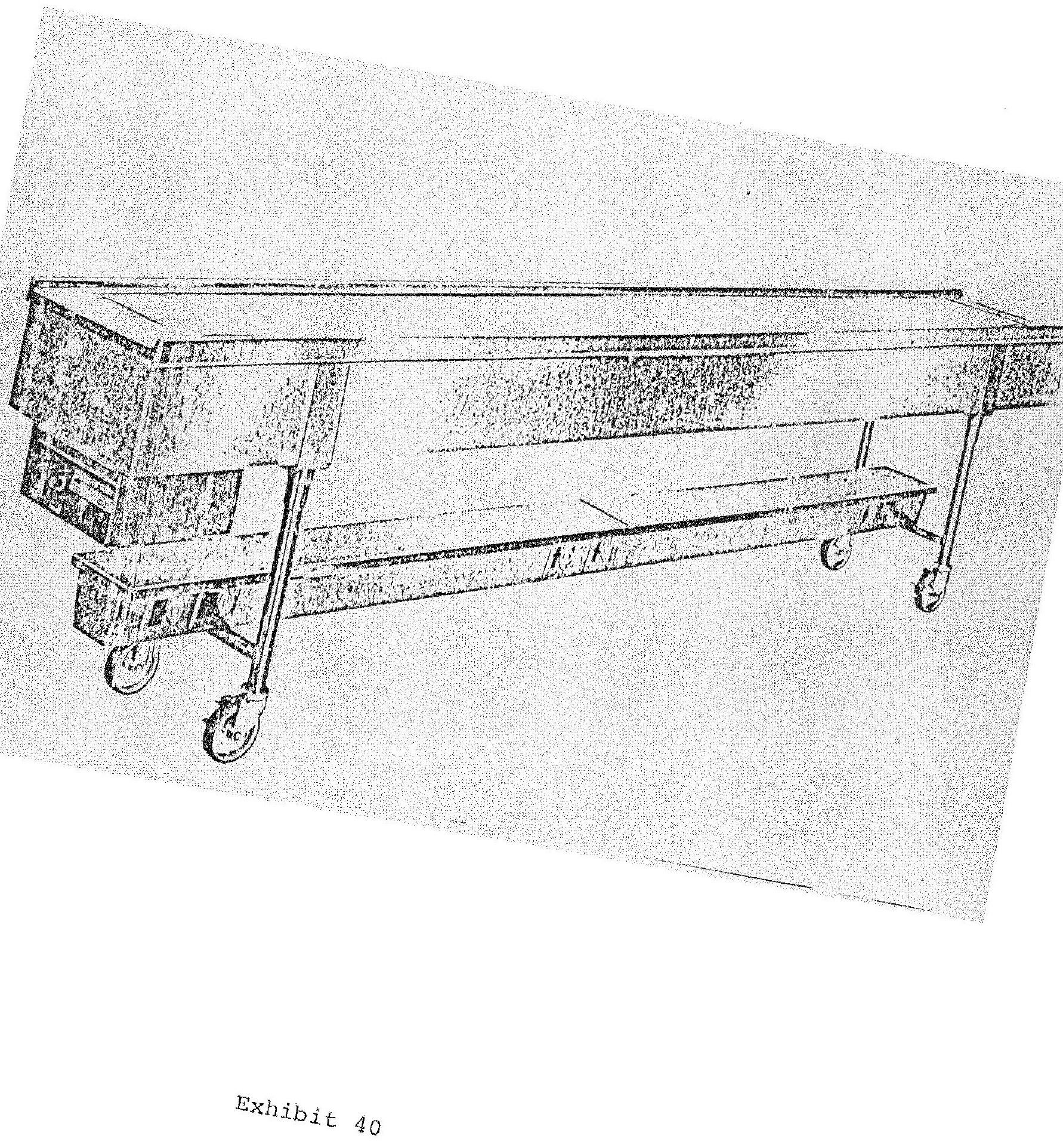




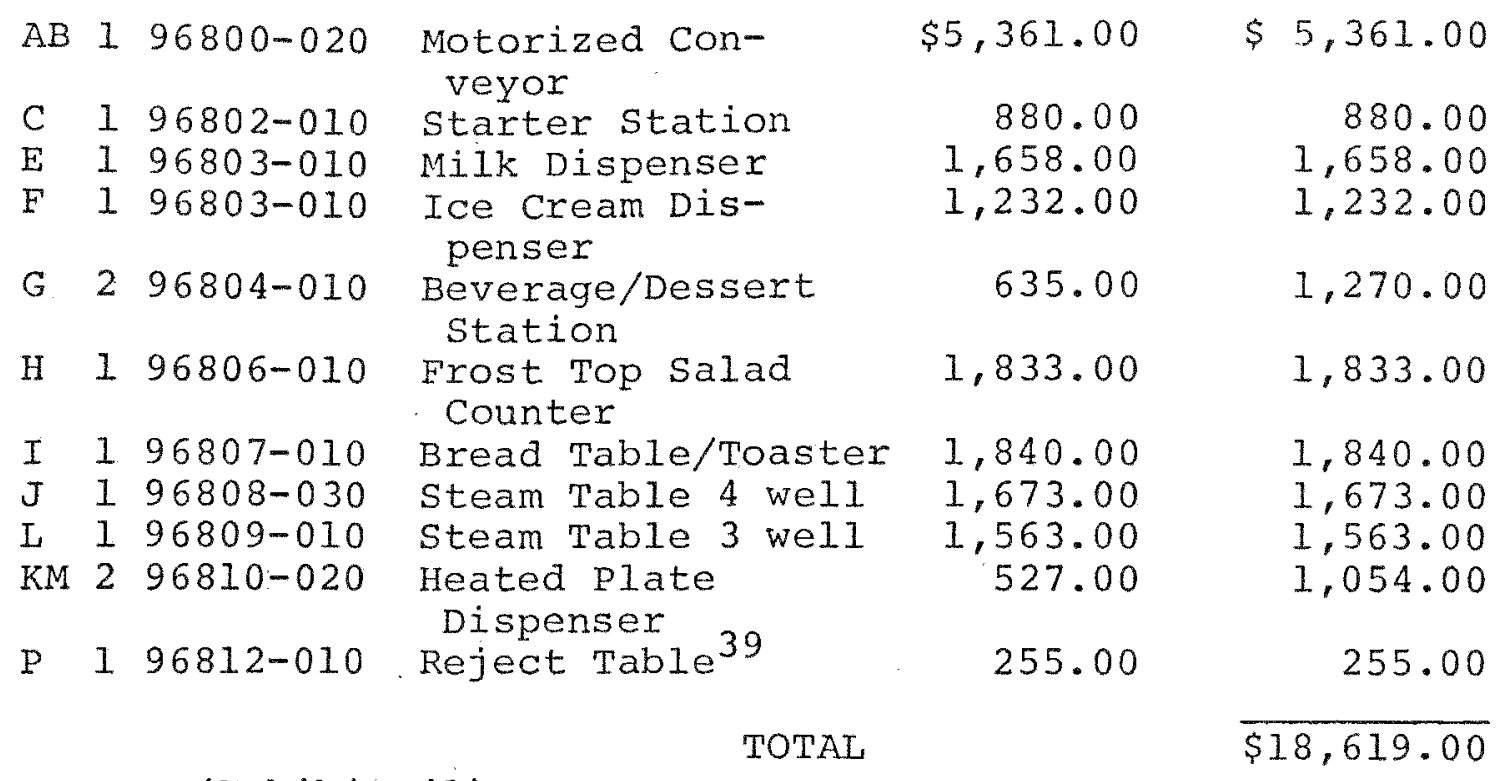

(Exhibit 41)

The Tray Type Line

Space and labor have come to be very costly factors in the dietary service. Tray lines have become long and use too many people. The tray type line was developed to save space and labor. This concept is different from the straight conveyor type line in that it is a motorized chain driven mechanism onto which stainless steel trays are attached. The trays travel in an oval racetrack pattern. An overshelf and frost top are in the center of the line. The support equipment is placed around the line as illustrated. (Exhibit 42) The line is only 14 feet 7 inches long and can be used for either hot or cold plating. An electrical raceway can be attached for plugging in support equipment.

39Dietary Products Division, American Hospital Supply Corporation, Catalog, MCGaw Park, IL, 1975. 

The line had been designed originally for the in-flight feeding commissaries of airlines but was later adapted for hospital food services. Six people can operate this line with ease. (Exhibit 43)

The line, marketed to hospitals by Dietary products, Division of American Hospital Supply Corporation, under the name of Mini-ouick, is sold with the following components:

1 starter station

1 ice cream cooler

24 well steam tables

1 coffee station

I tray line

Complete: $\$ 21,080.00^{40}$

Variations can be made for cold plating.

No matter what tray line is used in a hospital, some considerations must be made. They are:

Flow patterns

Fatigue factors

Back-up supplies

Space

Labor

Time and motion studies

quantity of trays to be made up

Time needed to serve

40 Dietary Products Division, American Hospital Supply Corporation, Catalog, MCGaw Park, IL, 1975. 

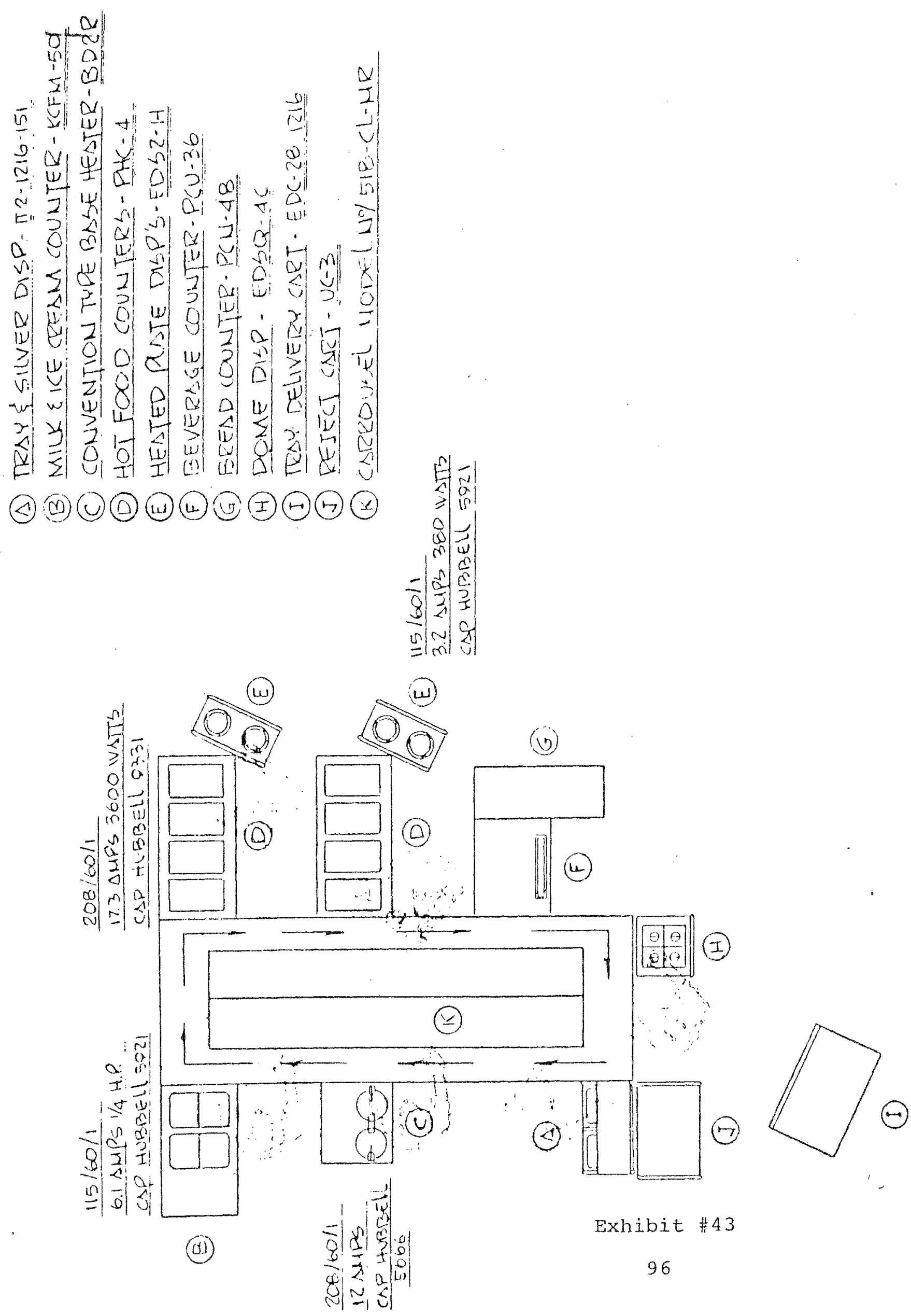
The tray make-up line has become a precision operation like that of a factory. Recently a German company, Voss, has computerized the tray make-up Iine. They use a computer to Iight Iights at the different stations to indicate which items are to be put on the tray. It also has a read-out at the end so the checker can check the tray rapidly. This is just one of many innovations which have come to the tray make-up 1ine. More are expected in the future and could possibly lead to the development of a fully automated line. 
Conveyors

Ndamation, Inc.

Alco/Shelcon, Inc.

American Pipe and Steel Corporation

Arista Metalcrafts, Co.

Bastain-Blessing Co., Div. of Golconda Corporation

S. B1ickman, lnc.

Cadily Corporation of America

Container Development Company

Crimsco, Inc.

lallas Sheet Metal Works, Inc.

Ekco Products, Inc,

The Fabricators, Div, of Dallas Shect Metal Works, Inc.

Food Equipment Corporation

Greitzer, Inc.

The Hobart MEg. Company

Hollymatic Corporation

Insinger Machine Company

Kitchen Equipment Corporation

l'aul E. Kraeft, Inc.

Lincoln Food Scrvice Systems

Metzgar Conveyor Co., Inc.

Precision Metal products, Inc.

Servco Equipment Company

Southern Equipment Company

Southern Stainless Equipment Company

Tafco, Inc.

Theodore Equipment Corporation

Traycon Manufacturing Company

Theodore Equipment Corporation

Traycon Manufacturing Company

Tyson Netal. Products Company

Inited Service Equipment Company

Washington Equipment Company, Inc.

N. Wasserstrom and Sons, lnc.

Wear-Ever Food Service Equipment Company

Steam Tables

Acme Equipment Company

Alvance lood Service, liquipment, Inc:

Alco/Sholloy Manufacturing Company

Alpine Store Equipment Corporation

Anderson-lully Company

Arista Metalcrafts Company

Atlanta Kitchen Fabricators, Inc.

Bastian-Blessing Company, l)ivision of Golconda Corp. Carter-llofimann Corporation 
Cradle Queen Barbeque Corporation

Crimsco, Inc.

Curtis liquipment Corporation

Dallas Sheet Metal Works, Inc.

Dean Products, Inc.

Delfield Division, C1ark Equipment Company

Duke Manufacturing Coripany

Dunhill Food Fquipment Corporation

Epco - Division of Standard International

Everfrost Inc.

Food Equipment Corporation

Foremost Industries, Inc.

Frankline Products Corporation

Grand Rapids Cabinet Company

Ilolderle brothers, Inc.

llot Food Boxes, Inc.

Illinois Range Company

Kitchen Equipment Corporation

Stanlcy Knight Corporation

Leitner Equipment Company

Levelator Corporation

Lincoln Food Service Systems

Low Temp Manufacturing Company, Ine.

B. L. Migali, Inc.

Origina1 Metal Manufacturing Company

Pase Equipment Company, Inc.

precision Metal products, Inc.

Progressive Corporation

Seco Company, Inc.

Sefi Fabricators, Inc.

Servco Rquipment Company

Southern Stainless Equipment Company

Star Metal Corporation

Sterling Notalware Company

Sylvan Stajnless products Inc.

Tafco, Inc.

Terriss Consolidated Ind.

Theodore Equipment Company

T'yson Metal products Company

vitro products Inc.

Washington Iiquipment Company, Inc.

N. Wasserstrom and Sons, Inc.

Wells Manulacturing Corporation

Cold llolding Tables

Alco/Shelley Manufacturing Company

Mrista Metalcrafts Company

Mrizona Booth and Fixture Manufacturing Corporation

Carter-loffinan Corporation

Crimsco, Inc. 


\section{Cold llolding Tables (continued)}

Curtis liquipment Corporation

Dallas Sheet Metal Works, Inc.

Dean Products, Inc.

Delfield Division, CIark Equipment Company

The Fabricators, Division of Lallas Shect Motal Works

Food Equipment Corporation

Foremost Industries, Inc.

Frigid Igloo Manufacturing Corporation

Glenco Refrigeration Corporation

Grand Rapids Cabinet Company

llolderle Bros., Inc.

llot Food Boxes, Inc.

Kitchen Fquipment Corporation

Stanley knight. Corporation

Lincoln Food Service Systems

B. E. Migali Inc.

Original Metal Manufacturing Company

Precision Metal Products Inc.

Progressive Corporation

Servco Equipment Company

Southern Equipment Company

Southern Stainless Equipment Company

Star Metal Coxporation

Storling Metalware Company

'Tafco, Inc.

Theodore Equipment, Corporation

Tyson Metal Products, Inc.

United Store Fixture Manufacturing Company

Washington Equipment Company, Inc.

N. Wasserstrom and Sons, Inc.

\section{Work Counters}

Acme Equipment Company

Advance Food Service Equipment, Inc.

Acro Manifacturing Company

Alco/Metal Masters of Baltimore

Alpine Store Fquipment Corporation

Amtekco lndustries, Inc.

Anderson-Tully Company

Arista Metalcrafts Company

Atlanta Kitchon Fabricators, Inc.

Bally Block Company

Bastain-Blessing Company, Div. of Colconda corporation John Boos and Company

Cradle Qucon Barboque Corporation

Curtis lipuipment Corporation

Dallas Sheot Metal Works, Inc.

Duke Manufacturing Company

Dunhi1l. Food liquipment Corporation

Lastorn Steel Rack Company

Ekco products, Inc. 
Epco - Jiv. of Standard International

The Fabricators, Div. of Dallas Sheet Netal Works, Inc. Falcon products, Inc.

Fearless Dishwasher Company, Inc.

Food Equipment Corporation

Foremost Industries, Inc.

Georgia Maplo Block Company, Inc.

Gordon Enterprises, Inc.

Grand Rapids Cabinet Company

llolderle Brothers, Inc.

Hot Food Boxes Inc.

Illinois Range Company

Kitchen Equipment Corporation

Stanley knight Corporation

lakeside Manufacturing, Ine.

Leitner Equipment Company

Levelator Corporation

Lincoln Food Service Systems

Jow Temp. Manufacturing Company, Inc.

Lyon Metal Products, Inc.

Michigan Maple Block Company

B. E. Migali, Inc.

Missouri- Equipment Company

Mobile Master - Div. of Stainless and Nlum Specialty Company, Inc.

Original Motal Manufacturing Company

Pase Equipment Company, Inc.

Precision Metal products, Inc.

Progressive Corporation

Quality Maple Block Company, Inc.

R. II. J. Products, Inc.

Rob Roy Division, The Vecta Group, Inc.

Sani-Serv

Seco Company, Inc.

Servco liquipment Company

Southern Stainless Equipmont Company

Stanley Knight Corporation

Sterling Metalware Company

Tafco, Inc.

Terriss Consolidated Ind.

Theodore Equipment Corporation

Tyson Motal products Company

Washington Equipment Company, Inc.

N. Wasscrstrom and Sons, Inc.

Wear-Bver llood Sorvice Lquipment Company

Wilder Manufacturing Company, Inc.

Wyott Corporation 


\section{CHAPTER IV}

TRANSPORTING

After the trays have been assembled, they must be transported to the patient's floor as quickly as possible. There are several different methods of achieving this. They are:

I Dumb Waiter: A tray conveyor at the end of the production line which runs in a shaft to the patient's floor where they are unloaded and delivered to the patients. (Exhibit 44)

II Cart-veyor: An elevator made to carry a special cart built to fit into it to the patient's floor. The cart is loaded in the central kitchen then transported to the floors and used to distribute trays to the patients and return the dirty trays to the kitchen. (Exhibit 45)

II Regular elevator: This standard elevator can hold one or more carts, hot or cold. They are used for transporting people when not beine used for the purpose of the dietary department. They are best when they are located near the dietary department but often are the same elevators that visitors use. 


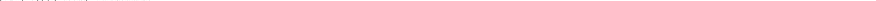




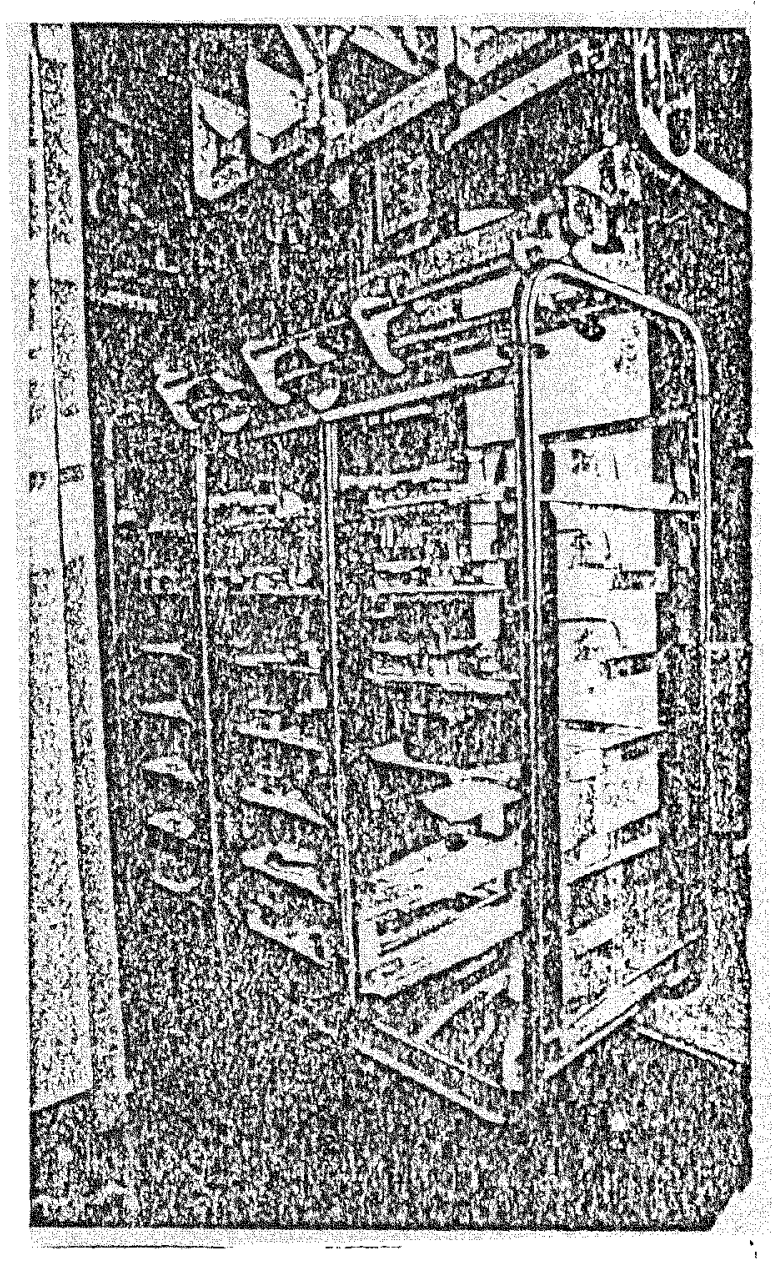

Exhibit \#45 
IV Rail systems: Rails built into the walls of the hospital onto which special carts are attached. The carts are programmed as to which floor they are to go. The cart can travel vertically or horizontally in the system until it gets to the station where they are to be delivered. There they are picked up by the personnel who are to deliver the tray. The carts are pushed on its roller on the patient's floor.

$V$ Self Driven Carts: Carts with a special indicator are programmed to deliver the cart with no one pushing it to the proper patient's floor. The indicator follows a metal strip embedded in the floor until it gets to its destination.

VI Hallways and ramps: Certain hospitals are built on one level with long ramps and hallways leading to the different wards. Manual or motorized carts are used to distribute the trays.

Decisions as to which of these methods is the best, must take place in the initial planning of the hospital. Future growth and expansion should be taken into consideration. Since most of the systems are built into the hospital, changing it after the hospital is built is a very expensive proposition. 
Things that must be considered are:

Time from the kitchen to the ward

Safety

Sanitation

Maintenance costs

What others are using the system being evaluated

Heat maintenance system and its effectiveness

Costs

Adaptability to change over the years Labor needed 


\section{SUIMARY}

Temperature maintenance delivery systems and assembly systems as reviewed have shown very little change in concept. The major changes which have taken place are improvements upon the basic concept, like making the pellet system a unitized system. The refining of the systems have made the systems workable, saved labor, and improved service. Some of the concepts that seemed to have been fading away are now enjoying a rebirth. This is true of the decentralized system. This seems to exemplify George Orwell's theory in Animal Farm, that all revolutions run in cycles. Hopefully, each new revolution in the temperature maintenance delivery systems and assembly systems are for the improvement of the systems.

Improvements are made by using sound management techniques and inventiveness. Considerations of the type of facility, standard of food service, type of service, size, sanitation, maintenance, esthetics, and financial constraints must be made. 
The costs figured in this study revealed the

following information:

Temperature Maintenance Systems (cost per bed for the 200 bed hospital)

$\begin{array}{lc}\text { Insulated Containers } & \$ 113.43 \\ \text { Aladdin } & \$ 110.37 \\ \text { Dri-Heat } & \$ 125.00 \\ \text { Unitized Pellet } & \$ 107.78 \\ \text { Hot and Cold Carts } & \$ 183.81 \\ \text { Unitray } & \$ 133.80 \\ \text { Bulk Food Carts } & \$ 135.30 \\ \text { Microwave Galley } & \$ 339.00 \\ \text { Other Galleys } & \$ 300.00\end{array}$

Tray Assembly Lines

$\begin{array}{ll}\text { The Manual Slide Line } & \$ 8,501.00 \\ \text { The Skate Wheel and } & \$ 10,662.00 \\ \text { Roller Conveyor } & \$ 18,619.00 \\ \text { The Belted Line } & \$ 21,080.00\end{array}$

The figures are just examples of a typical set up. Many variations can be incorporated in the layout and design of any hospital.

After studying all of the temperature maintenance and delivery systems, it became apparent the old decentralized system with bulk food cart is dead or dying. Centralization of assembly gives better control and saves labor. A new decentralized system is developing. It is a combination of centralized tray assembly with decentralized end heating. 
The unitized pellet systems, because of their heat retention, labor savings and cost would be the best heat maintenance system for hospitals using a centralized assembly system. This is especially true when smaller, large capacity carts are utilized with a high speed transporter. The size of the hospital does not affect the system; it is as equally good in a small hospital as in a larger one, if the transporter gets the carts to the patients" floor within 15 minutes.

Some hospitals do not have a good transporting system or have grown horizontally, making it impossible for the unitized pellet systems to be effective heat maintainers. In such incidents, central assembly is recommended with microwave end heating. Microwave heating has to be perfected to where all the guesswork is taken out. It doesn't tie the hospital to special china sizes or expensive carts. Ideally, a unitized pellet system could be used with the microwave system by putting a pellet heater in each pantry or galley. This would give the best of two worlds, food end heated near the patient with a constant heat source to maintain the temperature as the patient eats all of the courses. This is a little more expensive, but it does provide the best possible service at the present time.

Manual slide or skate wheel conveyors are good for hospitals under 100 beds. For hospitals over 100 beds, a 
motorized conveyor is best suited. They are more functional and help motivate the employees because the workers feel they must keep up with the line. With the cost of building rising, space is valuable. This makes the tray type line more practical even though it is more expensive. It occupies less space than a belted line which will hold down the construction cost.

The trend in heat maintenance and assembly systems is toward more compact, labor saving, easy to handle and more economical systems.

The challenge that faces the hospital food service industry is how to best utilize the plan systems that are available and how to develop new systems that will provide better service more economically. 
Exhibits Courtesy of the Following Companies:

Company

Aladdin Synergetics, Inc.

Blickman Health Industries, Inc.

Brunor, Inc.

Caddy Corporation of America

Cambro, Inc.

Crimsco, Inc.

Dietary Products, Division American Hospital Supply Corp.
Exhibit No.

11

22

27

43

2

6

44

4

29

34

I

3

5

8

9

10

12

13

15

16

17

18

19

20

21

24

26

30

31

32

33

41

42

Dinex Division, King Seeley Thermos Co. 7

Dri-Heat Division, Franklin Products Corp. 14

Food Management Magazine 45 
Exhibits Courtesy of the Following Companies: (cont'd.)

\section{Company}

Foster Refrigeration Corp.

Litton systems, Inc.

Precision Metal Products, Inc.

38

39

40

Shelley Manufacturing Co. 36

United Service Equipment 
Aladdin Synergetics, Inc., A Subsidiary of Aladdin Industries, Inc., "From Kitchen to Patient NonStop," Nashville, TN, 1975.

Aladdin Synergetics, Inc., A Subsidiary of Aladdin Industries, Inc., Contract, Nashville, TN, 1975.

American Association of Hospital Consultants, Functional Planning of General Hospitals, MCGraw-Hill Book Co., New York, 1969.

AMF, Food Service Division, Catalog, Essex, CT., 1974.

Barker, E.: What the budget for the dietary service of Flower Hospital. Modern Hospital, 23:160 (Aug) 1924 .

Bastian-Blessing, Inc., Catalog, Grand Haven, Michigan, 1975.

Beesley, Charles, Critical Analysis of the Aladdin system, Sweetheart Cup Division, Maryland Cup Corp., Owing Mills, MD, 1972 .

Blickman Health Industry, Inc., Catalog, Fairlawn, NJ, 1975. Brunor, Inc., Catalog, Miami, FL, 1974. Bucksco, Catalog, Quakertown, PA, 1975.

Caddy Corporation of America, Subsidiary of Rockaway Corp., Catalog, Pittman, NJ, 1975.

Cambro, Inc., Catalog, Huntington Beach, CA, 1975.

Carter Hoffman Corp., Catalog, Mundelein, IL, 1975.

Commercial Kitchen and Dining Room, U.S. Industrial

Publications, Inc., Stamford, CT, Fall, 1975.

Crescent Metal Products, Inc., Catalog, Cleveland, OH, 1975. Crimsco, Inc., Catalog, Kansas City, MO, 1975.

Dietary Products Division, American Hospital Supply Corp., Catalog, MCGaw Park, IL, 1975.

Dietary Products Division, American Hospital Supply Corp., Product Information Manual, MCGaw Park, IL, 1975. 
Bibliography, cont'd.

Dinex Products, Division King Seeley Thermos Co., Catalog, Norwich, CT, 1974.

Dri-Heat Division, Franklin Products, Corp., Catalog, Hudson, NY, 1975.

Essays on History of Nutrition and Dietetics. The American Dietetic Association, Chicago, IL, 1967.

Food Management, a Harcourt Brace Jovanovich Publication, New York, NY, Oct., 1975.

Food Service Manual for Health Care Institutions, American Hospital Association, Chicago, IL, 1972.

Food Service Marketing, EIP, Inc., Madison, WI, May, 1974.

Foodservice Equipment Dealer, Product Directory, Cahners Publishing Co., Chicago, IL, 1974.

Foster Refrigeration Corp., Catalog, Hudson, NY, 1975.

General Electric Co., Catalog, Chicago Heights, IL, 1975.

H. B. E. Leasing Corp., Subsidiary of H. B. E. Corp., Catalog, Philadelphia, PA, 1975.

Hospitals, American Hospital Association, Chicago, IL, sept. 1, 1963.

Hospital Dietary Service, U.S. Department of Health, Education, and Welfare, Washington, DC, 1966.

Hobart Corp., Catalog, Troy, Ohio, 1975.

Institutions/Volume Feeding Magazine, Cahners Publishing Co., Chicago, IL, 1975.

Journal of the American Dietetic Association, Chicago, IL, $9,464,1934$.

Kotschevar, L. H., Food Service for the Extended care Facility, Institutions/Volume Feeding Magazine, Cahner Books, Boston, MA, 1973.

Kotschevar, L. H. and Terrell, M. E., Food Service Layout and Equipment Planning, John Wileys and Sons, New York, NY, 1971. 
Bibliography, cont'd.

Kotschevar, L. H. and Terrell, M. E., Food Service Planning, John Wiley and Sons, Inc., New York, NY, 1971.

Lakeside Manufacturing, Inc., Catalog, Milwaukee, WI, 1975.

Lincoln Food Service Systems, Catalog, Fort Wayne, IN, 1974.

Litton Microwave Cooking Products, Hospital Patient Feeding Systems, Minneapolis, MN, 1975.

Market Forge, Catalog, Everett, MA, 1975.

Ogden, Ruth F.; Wenberg, Burness G., Introduction to Health Professions. The C. U. Mosly Co., St. Louis, MO, 1972 .

Orwell, George, Animal Farm: A Fairy Story, New American Library, New York, 1946.

Philbrick, M. H., Pioneering in Dietetics, Journal of American Dietetic Associates, H12;1301, Chicago, IL, 1936 .

Pinkert, Michael s., The Ready Foods System for Hoalth Care Facilities, Institutions/Volume Feeding Magazine, Chicago, IL, 1973.

Precision Metal Products, Inc., Catalog, Miami, FL, 1975.

Precision Metal Products, Hospital Patient Feeding Systems and Hi-Speed Compact Tray Make-up Systems, Miami, FI, 1974 .

Savory Equipment, A Division of Alco Food Service Equipment Co., Catalog, Neptune, NJ, 1974.

Seco Products, McGraw-Edison Co., Catalog, St. Louis, MO, 1975 .

Serv-O-Lift Corp., Catalog, Dorchester, MA, 1975.

Shelcon, Inc., A Division of Alco Food Service Equipment Co., Catalog, Alhambra, CA, 1974.

Shelley Manufacturing Co., A Division of Alco Food Service Equipment Co., Miami, FL, 1975.

SiLite, Inc., Catalog, Chicago, IL, 1975.

Star Metal Corp., Catalog, Philadelphia, PA, 1974. 
Bibliography, cont'd.

Stoker, John, Food Service Industry and Institutions,

Wm. C. Brown, Co., Dubuque, IA, 1973.

Traulsen and Co., Inc., Catalog, College Point, NY, 1975.

United Service Equipment Co., Catalog, Murfreesboro, TN, 1975.

Victory Metal Manufacturing Corp., Catalog, Plymouth Meeting, PA, 1975.

Voss of America, Inc., Catalog, Baltimore, MD, 1973.

N. Wasserstrom and Sons, Inc., Catalog, Columbus, OH, 1974.

Wells Manufacturing Corp., Catalog, San Francisco, CA, 1974 .

West, Bessie Brooks; Wood, Levelle; Harger, Virginia F., Food Service In Institutions. John Wiley \& Sons, Inc., NY, 1966. 